\title{
Neurofibromatosis type I-associated tumours: Their somatic mutational spectrum and pathogenesis
}

\author{
Sebastian Laycock-van Spyk, Nick Thomas, David N. Cooper and Meena Upadhyaya* \\ Institute of Medical Genetics, School of Medicine, Cardiff University, Cardiff, UK \\ *Correspondence to: Tel: +44 2920 744081; Fax: +44 2920 746551; E-mail: Upadhyaya@cardiff.ac.uk \\ Date received (in revised form): 23rd May 2011
}

\begin{abstract}
Somatic gene mutations constitute key events in the malignant transformation of human cells. Somatic mutation can either actively speed up the growth of tumour cells or relax the growth constraints normally imposed upon them, thereby conferring a selective (proliferative) advantage at the cellular level. Neurofibromatosis type-I

(NFI) affects I/3,000-4,000 individuals worldwide and is caused by the inactivation of the NFI tumour suppressor gene, which encodes the protein neurofibromin. Consistent with Knudson's two-hit hypothesis, NFI patients harbouring a heterozygous germline NFI mutation develop neurofibromas upon somatic mutation of the second, wild-type, NFI allele. While the identification of somatic mutations in NFI patients has always been problematic on account of the extensive cellular heterogeneity manifested by neurofibromas, the classification of NFI somatic mutations is a prerequisite for understanding the complex molecular mechanisms underlying NFI tumorigenesis. Here, the known somatic mutational spectrum for the NFI gene in a range of NFI-associated neoplasms including peripheral nerve sheath tumours (neurofibromas), malignant peripheral nerve sheath tumours, gastrointestinal stromal tumours, gastric carcinoid, juvenile myelomonocytic leukaemia, glomus tumours, astrocytomas and phaeochromocytomas — have been collated and analysed.
\end{abstract}

Keywords: NFI, somatic mutations, germline mutations, pathogenesis, tumorigenesis, tumour, benign, malignant

\section{Introduction}

Neurofibromatosis type 1 (NF1) is a common autosomal dominantly inherited tumour predisposition syndrome affecting $1 / 3,000-4,000$ individuals worldwide. ${ }^{1,2}$ NF1 manifests a variety of characteristic features that include: hyperpigmentary abnormalities of the skin (café-au-lait macules and inguinal/axillary freckling), iris hamartomas (Lisch nodules) and the growth of benign peripheral nerve sheath tumours (neurofibromas) in the skin. Neurofibromas display many different subtypes and are associated with a variety of different clinical complications. Cutaneous neurofibromas are present in almost all adult NF1 patients. ${ }^{3}$ Plexiform neurofibromas (PNFs), a more diffuse type of tumour, are present in 30-50 per cent of NF1 patients, and some $10-15$ per cent of these benign tumours are transformed to malignant peripheral nerve sheath tumours (MPNSTs), the main cause of morbidity in NF1. ${ }^{4}$ Other NF1-associated clinical features include: skeletal abnormalities, such as tibial bowing or pseudoarthrosis; skeletal and orbital dysplasia; ostopenia/osteoporosis; aqueduct stenosis; macrocephaly; pectus excavatum; short stature; cardiovascular malformations; learning difficulties; and attention deficit disorder. ${ }^{1,5}$

Cancer represents the transformation of a cell whose growth is normally tightly controlled into one that is no longer under strict regulation, 
allowing the cell to multiply uncontrollably and even metastasize. This dramatic alteration in cellular control arises as a consequence of the accumulation of genetic and epigenetic changes: activated oncogenes speed up cell growth through the acquisition of gain-of-function mutations, whereas tumour suppressor genes (TSGs) promote progression by acquiring loss-of-function mutations. TSGs typically encode proteins involved in growth regulation, apoptosis initiation, cellular adhesion and DNA repair. In accordance with Knudson's two-hit hypothesis, ${ }^{6}$ both alleles of a TSG must be inactivated for cellular transformation to occur. Typically, a patient will inherit a germline mutation in one TSG allele; a second-hit or somatic mutation then occurs post-fertilisation, thereby inactivating the remaining wild-type allele. Somatic mutation is thus a key event in cancers associated with TSG inactivation. Upon transformation, a cell may acquire many additional somatic mutations elsewhere in the genome, a few of which actively encourage tumour progression, designated as 'driver mutations', while most occur simply because of the increased number of cell replications and are usually of unknown biological consequence and so are designated as 'passenger mutations'.

The NF1 gene encodes neurofibromin, a negative regulator of the Ras/mitogen-activated protein kinase (MAPK) pathway. NF1 is a TSG and, consistent with Knudson's two-hit hypothesis, most patients carry (in all their cells) both a normal and a dysfunctional NF1 gene copy - the latter harbouring the inherited (germline) mutation. It may be inferred that any tumours that arise will have acquired a second, somatic 'hit' that inactivates the normal NF1 allele, resulting in a complete loss of functional neurofibromin; a double hit $\left(\mathrm{NF}^{-/-}\right)$ is critical for NF1 tumorigenesis to occur. ${ }^{8,9}$ The question as to why only a few of these benign tumours eventually go on to become malignant, however, is still puzzling. Consistent with a central role for neurofibromin in cellular function, recent cancer genome sequencing studies have found that somatic NF1 gene mutations occur not only in association with NF1, but also in a number of other common cancers. ${ }^{10-16}$
In the context of NF1, few genotype-phenotype correlations are evident. Indeed, marked intrafamilial variation in terms of the clinical phenotype is common. ${ }^{5,17}$ The existence of such families is perhaps an indication of the importance of the second hit, since differences in the type and timing of somatic NF1 mutations may help to explain the variability in patient phenotype. ${ }^{18}$ An appreciation of the spectrum of somatic mutations in NF1-associated tumours is therefore essential if we are to understand the molecular pathways involved - itself a prerequisite for improvements in clinical treatment and the development of new therapeutics. This paper attempts to collate and review the spectrum of somatic NF1 mutations so far reported in NF1-associated tumours, with a view to assessing how they may serve to induce tumour growth and whether or not any genotype-phenotype correlation may be discerned

\section{The NFI gene: Structure and function}

The NF1 gene spans 283 kilobases $(\mathrm{kb})$ of genomic DNA at $17 \mathrm{q} 11.2^{19}$ and contains 61 exons. ${ }^{3,20}$ Neurofibromin, the $327 \mathrm{kDa}$ protein encoded by the NF1 gene, is translated from a $12 \mathrm{~kb}$ messenger mRNA transcript, and has a number of alternative isoforms $^{21-24}$ (reviewed by Upadhyaya ${ }^{25}$ ). Neurofibromin contains 2,818 amino acids and is expressed at low levels in all cells, with higher levels in the nervous system. It functions as a negative regulator of active Ras, and of the associated Ras/MAPK signalling pathway. Neurofibromin contains a Ras-specific GTPase activating protein (GAP)-related domain which interacts directly with Ras, resulting in a conformational change that greatly stimulates the intrinsic GTPase activity of the Ras protein, thus significantly accelerating the conversion of the active GTP-bound form of Ras into its inactive GDP-bound form and effecting a net decrease in overall mitogenic signalling in the cell. As the Ras/MAPK cascade is critical for the control of cellular growth and differentiation, a lack of functional neurofibromin results in the constitutive 
activation of this central signalling pathway and in unregulated cell growth. ${ }^{26}$

\section{NFI tumour biology}

A variety of benign and malignant tumours are associated with NF1 and all involve tumorigenesis of neural crest-derived cells. Several murine models of neurofibromatosis have both successfully recapitulated much of the NF1 human phenotype and shown that NF1 is indeed a classical TSG. ${ }^{27,28}$

Neurofibromas exhibit extensive cellular heterogeneity, being composed of hyperproliferative Schwann cells (SCs), fibroblasts, mast cells and perineural cells. The SCs have been identified as the initiating cell type in neurofibromas and it is only in these cells that the NF1 gene becomes biallelically inactivated. ${ }^{29}$ SCs are also the target for various growth factors known to stimulate neurofibroma formation and growth. What is still not known, however, is the precise cell type within the SC cell lineage in which the somatic mutation occurs, the cell type which subsequently precipitates neurofibroma growth.

Cutaneous neurofibromas are thought to arise from skin-derived precursor cells $(\mathrm{SKPs})^{30}$ and these cells may well be under hormonal control, since most such tumours develop only during puberty. ${ }^{31}$ Further, an increase in tumour size and number has also been noted during pregnancy, with some evidence for a postnatal decrease in tumour size. ${ }^{32,33}$ Almost all PNFs appear congenitally and it is thought that they are induced by a somatic NF1 mutation in SC precursors within the embryonic gestational window of $12.5-15.5$ days. ${ }^{34}$ It may be that this second hit does not render the SC precursor tumorigenic, but instead induces aberrant axonal segregation. ${ }^{35}$ The extracellularly expressed transmembranal guidance protein, Sema4F, is strongly downregulated in neurofibromas and it has been suggested that this somehow indirectly promotes SC proliferation by rendering these cells more responsive to environmental signals, possibly through inhibition of axonal re-attachment. $^{36}$ In this way, the disruption of normal SC axonal interactions leads to neurofibroma development. An $N F 1^{-/+}$haploinsufficient cellular environment is also considered necessary, probably because of the growth advantage conferred by the signalling deficiency due to reduced neurofibromin levels. Indeed, Le et al. ${ }^{30}$ found that NF1 inactivation is necessary, although not sufficient, for neurofibroma formation, highlighting the importance of the tumour microenvironment. There is some evidence to indicate that the haploinsufficiency $\left(N F 1^{-/+}\right)$of the other supporting cells (fibroblasts, mast cells and perineurial cells) cooperates in neurofibroma development. ${ }^{37}$ Additionally, it has been shown that $N F 1^{-/+}$haploinsufficient mast cells readily migrate into preneoplastic nerves, probably in response to Kit ligand, which exhibits four-fold increased levels in nullizygous SCs as compared to normal SCs. ${ }^{38,39}$ The molecular mechanisms underlying both PNF and cutaneous neurofibroma formation are becoming clearer, although the major details are still lacking. It would appear that the key to understanding neurofibroma formation lies in the elucidation of the precise molecular interactions of the haploinsufficient tumour microenvironment within the initial cell type harbouring the biallelically inactivated NF1 gene.

\section{NFI-associated tumours}

\section{Cutaneous neurofibromas and PNFs}

Neurofibromas are a characteristic feature of NF1 and have a diverse clinical presentation. They are classified as grade 1 tumours by the World Health Organization; they have multiple forms and may affect nerves in any body location. Tumours derived from skin sensory nerves are designated dermal or cutaneous neurofibromas, and usually present as discrete tumours that remain associated with a single nerve ending. Approximately 20-50 per cent of cutaneous neurofibromas exhibit loss of heterozygosity (LOH) at the NF1 locus and the majority of these lesions appear to be due to mitotic recombination. ${ }^{40-42}$ Tumours associated with larger nerves within the skin may spread within the dermis and appear as a diffuse mass. PNFs are much larger tumours, usually associated 
with major nerve trunks and nerve plexi. They are generally slow growing, may develop at both internal and external body locations and can often result in major disfigurement. PNFs occur in some 30-50 per cent of patients with NF1 and, although these tumours generally remain benign, some neurological impairment may result from their growth. Approximately $10-15$ per cent of PNFs may become malignant.

While the genetic basis of neurofibroma development is still not fully understood, biallelic NF1 inactivation does seem to be required, as all tumour cells harbour both a constitutional and a somatic NF1 gene mutation. ${ }^{5}$ About 70 per cent of PNFs have been reported to display $\mathrm{LOH}$ at the NF1 locus; ${ }^{20}$ however, there is no obvious correlation between the type or location of germline NF1 mutations in NF1 patients and those of their somatic counterparts arising in their tumours. ${ }^{20}$

Another interesting, although as yet unexplained, observation is that a few patients mildly affected by NF1 who never develop any cutaneous neurofibromas or PNFs have been shown to carry the same germline NF1 mutation (c.2970-2972delAAT) namely, an in-frame 3-base pair (bp) deletion that leads to the loss of a methionine residue. ${ }^{3}$

\section{MPNSTs}

Cells derived from within some 10-15 per cent of PNFs may eventually undergo malignant transformation into an MPNST. MPNSTs are aggressive and highly invasive soft tissue sarcomas with an annual incidence of 0.16 per cent in NF1 patients, compared with only 0.001 per cent in the normal population, ${ }^{43}$ and with a lifetime risk of $8-13$ per cent in NF1 individuals $^{44,45}$ (reviewed by Upadhyaya ${ }^{4}$ ). This form of malignancy represents a major cause of morbidity and mortality in NF1. Malignant transformation usually appears to evolve from within a pre-existing PNF. $^{46}$ The distinction between benign PNFs and MPNSTs has been sensitively visualised by noninvasive $\left[{ }^{18} \mathrm{~F}\right]$-2-fluoro-2-deoxy-D-glucose positron emission tomography (FDG-PET) imaging, ${ }^{47}$ suggesting a potential role for FDG-PET-based noninvasive imaging in future diagnostic tests. The aberrant molecular pathways that underlie this malignant transformation are still largely unknown, and considerable effort is being directed towards elucidating the molecular defects involved.

NF1 patients carrying large (usually 1.4-megabase $[\mathrm{Mb}]$ ) genomic deletions (which remove the entire NF1 gene plus a variable number of flanking genes) have an increased risk of MPNST development in certain patient groups. ${ }^{48,49}$ Indeed, over 90 per cent of MPNSTs have been found to harbour large NF1 somatic deletions. ${ }^{20}$ More recently, significantly increased frequencies (relative to the general NF1 population) of PNFs, subcutaneous neurofibromas, spinal neurofibromas and MPNSTs have also been reported in association with molecularly ascertained 1.4 Mb type-1 NF1 deletions. ${ }^{50}$ The MPNSTassociated deletion breakpoints have been found not to involve the paralogous repetitive sequences that are involved in most germline NF1 deletions. ${ }^{18}$ The smallest common region of somatic deletion overlap is, however, restricted to approximately the same $\sim 2.2 \mathrm{Mb}$ interval that contains most of the genes deleted in recurrent constitutional NF1 deletions. ${ }^{51}$

Although it is clear that biallelic NF1 gene inactivation is required for transformation to occur, mutations at the NF1 locus are insufficient to explain the process of tumorigenesis, as most benign neurofibromas also exhibit such biallelic NF1 inactivation. The best evidence for the involvement of other loci relates to the tumour protein 53 gene (TP53), for which several different mutations have been found in MPNSTs that have not been reported in benign neurofibromas. ${ }^{4,20,52,53}$ Mice with heterozygous mutations in both their Nf1 and Tp53 genes developed malignancy, ${ }^{27,54}$ an indication, perhaps, that TP53 loss is critical to transformation. The homozygous loss of the cyclin-dependent kinase inhibitor $2 \mathrm{~A}$ gene $(C D K N 2 A)$, which encodes p16INK4A and p14ARF, has also been associated with NF1 malignancy. ${ }^{55-57}$ Another recent report has indicated that phosphatase and tensin homologue deleted on chromosome 10 gene (PTEN) dosage, and/or phosphatidylinositol 3-kinase/AKT8 virus oncogene cellular homologue (PI3K/AKT) pathway 
activation, may be rate-limiting steps in NF1 malignant transformation. ${ }^{58}$ As yet, however, no characteristic gene expression signature has been defined for MPNST development, although several cellcycle and signalling regulation genes: - cyclindependent kinase inhibitor (CDKN2A); tumour protein 53 (TP53); retinoblastoma 1 (RB1); epidermal growth factor receptor (EGFR); CD44 antigen (CD44); platelet-derived growth factor receptor alpha (PDGFRA); hepatocyte growth factor (HGF); proto-oncogene protein (C-MET) and transcription factor $(S O X 9)$ - are frequently deregulated. $^{4}$

Recent studies of the micro-RNA expression profile of MPNSTs have expanded the pathogenic spectrum associated with this tumour. For example, microRNA-34a (miR-34a) is downregulated in MPNSTs; this microRNA (miRNA) regulates many cell cycle genes and is also upregulated by p53, suggesting that TP53 loss would lead to downregulation of miR-34a and possibly several other miRNAs. This implies that this could be a critical event in malignant transformation. ${ }^{59}$ In similar vein, miR $-10 b$ has been reported to be upregulated in SCs from NF1 tumours, while miR-10b inhibition reduced MPNST cell proliferation, migration and invasion. ${ }^{60}$ NF1 mRNA is also a specific target for miR-10b, ${ }^{60}$ indicating that these miRNAs represent potential therapeutic targets.

\section{Spinal neurofibromas}

About 40 per cent of NF1 patients present with tumours involving their spinal nerves. This is especially marked in individuals affected with familial spinal neurofibromatosis (FSNF), a variant form of NF1 in which bilateral tumours involving multiple spinal nerve roots are often the only manifestation of NF1. ${ }^{61-63}$ Patients with FSNF have been reported to be significantly more likely to harbour missense or splice-site germline mutations compared with patients with classical NF1. ${ }^{64} \mathrm{~A}$ recent study of the NF1 locus found $\mathrm{LOH}$ in eight of 22 spinal tumours analysed, with most (75 per cent) of this $\mathrm{LOH}$ being due to mitotic recombination rather than genomic deletions. ${ }^{64}$

\section{Gastrointestinal stromal tumours (GISTs)}

GISTs are the most common mesenchymal tumours of the gastrointestinal tract. Although most GISTs harbour activating somatic mutations of KIT and PDGFRA, the absence of such mutations from NF1-associated GISTs (NF1-GISTs) is probably indicative of a different pathogenetic mechanism. In NF1, the majority (60 per cent) of GISTs develop in the small intestine, whereas sporadic non-NF1 GISTs mainly involve the stomach. ${ }^{65}$

Somatic NF1 mutations have been identified in the interstitial cells of Cajal (ICC) throughout the gastrointestinal tract and in NF1-GISTs lacking KIT or PDGRA mutations. ${ }^{66}$ Increased signalling through the Ras/MAPK pathway has also been shown to occur in NF1-GISTS, as opposed to sporadic GISTs. This would seem to indicate that a decrease in neurofibromin level, in the presence of normal c-KIT and PDGFRA levels, leads to tumour formation. It also suggests that NF1 haploinsufficiency is required for ICC hyperplasia, again demonstrating that, although a somatic NF1 mutation is absolutely necessary, it is not sufficient to permit tumorigenesis: additional genetic events required. These observations concur with Knudson's two-hit hypothesis. Somatic inactivation of the NF1 gene through gene deletion; intragenic deletion; and $\mathrm{LOH}$ through mitotic recombination have also been described. ${ }^{66,67}$

\section{Gastric carcinoid}

Gastric carcinoid tumours are associated with multiple endocrine neoplasia, atrophic gastritis and pernicious anaemia but are very rare in NF1. ${ }^{17}$ LOH at the NF1 locus has been demonstrated in a gastric carcinoid tumour derived from an NF1 patient. ${ }^{67}$

\section{Juvenile myelomonocytic leukaemia (JMML)} Young NF1 patients are at particular risk of developing JMML, ${ }^{68}$ a clonal haematopoietic disorder characterised by hypersensitivity (at least in vitro) to granulocyte-macrophage colony-stimulating factor (GM-CSF). Moreover, some 15-20 per cent of JMML patients harbour a somatic NF1 inactivating 
mutation, even though most exhibit no other NF1 symptoms. ${ }^{69}$ Patients may also carry inactivating mutations of other genes, with a recent study identifying that 70-80 per cent of mutations involve genes in the Ras/MAPK pathway, including one tyrosine-protein phosphatase non-receptor type 11 (PTPN11), neuroblastoma RAS viral oncogene homologue (NRAS), and $\mathrm{v}-\mathrm{Ki}$-ras2 kirsten rat sarcoma viral oncogene homologue (KRAS) as well as NF1 genes. ${ }^{70}$ Additional somatic mutations have also been reported in the casitas B-lineage lymphoma $(C B L)$ and additional sex combs-like 1 (ASXL1) genes. $^{71}$ In most cases, the NF1 gene is lost either via $\mathrm{LOH}$ or by compound heterozygous microlesions, ${ }^{72}$ which lead to a complete loss of neurofibromin and hyperactive signalling through the Ras/MAPK pathway. LOH may occur through 1.2-1.4 Mb interstitial deletions mediated by low copy number repeat (LCR) elements that flank the NF1 gene. ${ }^{73} \mathrm{LOH}$ through uniparental interstitial isodisomy (50-52.7 $\mathrm{Mb})$ of chromosome 17 through double mitotic recombination, in an as-yet-unknown initiator cell, has also been reported. ${ }^{72}$ The rarity of such events may indicate the existence of a selective advantage, conferred upon the $N F 1^{-/-}$cells, which might explain the propensity of NF1 patients to develop leukaemia. ${ }^{74}$

\section{Astrocytomas (ACs)}

Optic pathway tumours or ACs are found in $\sim 15$ per cent of paediatric NF1 patients, ${ }^{75}$ with the complete loss of neurofibromin evident in NF1-associated optic gliomas. ${ }^{76}$ Approximately 84 per cent of NF1-associated ACs also exhibit LOH in the NF1 region, with many tumours also exhibiting $\mathrm{LOH}$ of $17 \mathrm{p}$, suggesting the likely role of TP53 - or other 17p13-located genes - in AC formation. ${ }^{77}$ As with MPNSTs, biallelic somatic NF1 mutation in ACs is, again, apparently insufficient to induce transformation.

\section{Phaeochromocytomas (PCs)}

PCs are extremely rare tumours, with only one to six cases observed per million individuals. PCs develop from neural crest-derived chromaffin cells, and the tumour cells produce and release catecholamines, which cause hypertension and flushing. These are tumours of the adrenal medulla and are primarily associated with mutations of the Ret proto oncogene (RET), von Hippel-Lindau (VHL), succinate dehydrogenase complex, subunit B (SDHB), succinate dehydrogenase complex, subunit C (SDHC), and succinate dehydrogenase complex, subunit D (SDHD) genes, although $\mathrm{LOH}$ in the NF1 region, as well as $\mathrm{LOH}$ of other loci on both $17 \mathrm{q}$ and $17 \mathrm{p}$, have been observed. ${ }^{78,79}$

\section{Glomus tumours}

Glomus tumours are small $(<5 \mathrm{~mm})$, benign, but often very painful tumours that develop specifically within the highly innervated glomus body located at the end of each digit. These tumours appear to develop from $\alpha$-smooth muscle actin-positive cells that have undergone biallelic NF1 inactivation, resulting in increased Ras/MAPK activity. ${ }^{80}$ The somatic NF1 mutations often differ between glomus tumours, indicating highly specific tumorigenic events. Brems et al. ${ }^{80}$ have suggested that glomus tumours, although rare, should now be recognised as an integral component of the NF1 spectrum of disease.

\section{The somatic mutational spectrum of NFI-associated tumours}

A review of all published - and the authors' many unpublished - somatic NF1 alterations associated with NF1 tumours was undertaken to gain a better appreciation of NF1 tumorigenesis. As of July 2010, at least 577 different somatic NF1 gene changes had been reported in different NF1-associated tumours, with more than half $(323 / 577$; 56 per cent) corresponding to $\mathrm{LOH}$ in the NF1 gene region, some involving much larger regions of chromosome 17 (Table S1). The level of $\mathrm{LOH}$ detected also differs between cutaneous neurofibromas, PNFs and MPNSTs (40 per cent, 79 per cent and 85 per cent, respectively; Table 1). Table 2 provides the incidence of $\mathrm{LOH}$ in the other tumour types, where 
Table I: Contribution of $\mathrm{LOH}$ and NFI micro-lesions to the somatic NFI mutational spectrum in different types of $\mathrm{NFI}$-associated tumour

\begin{tabular}{|c|c|c|c|}
\hline Tumour type & LOH & Point mutations & Total \\
\hline Dermal neurofibroma & I 44 (40\%) & $211(60 \%)$ & 355 \\
\hline Plexiform neurofibroma & $67(79 \%)$ & $18(21 \%)$ & 85 \\
\hline Spinal neurofibroma & 7 (70\%) & $3(30 \%)$ & 10 \\
\hline MPNST & $55(85 \%)$ & $10(15 \%)$ & 65 \\
\hline Astrocytoma & $18(100 \%)$ & $0(0 \%)$ & 18 \\
\hline GIST/gastric carcinoid & $3(38 \%)$ & $5(62 \%)$ & 8 \\
\hline JMML & $18(95 \%)$ & I* $(5 \%)$ & 19 \\
\hline Phaeochromocytoma & $10(100 \%)$ & $0(0 \%)$ & 10 \\
\hline Glomus tumour & I (I4\%) & $6(86 \%)$ & 7 \\
\hline Overall & $323(55 \%)$ & $254(44 \%)$ & 577 \\
\hline
\end{tabular}

appropriate evidence has been obtained by multiplex ligation-dependent probe amplification (MLPA), fluorescence in situ hybridisation (FISH) etc; 78 per cent $(28 / 36)$ of cutaneous neurofibromas, 44 per cent $(11 / 25)$ of PNFs and 16 per cent $(5 / 31)$ of MPNSTs display $\mathrm{LOH}$ resulting from mitotic recombination. Some 79 per cent $(15 / 19)$ of the JMML samples that exhibited $\mathrm{LOH}$ appear to have lost the entire $17 \mathrm{q}$ arm through mitotic recombination, perhaps indicating a significant correlation with this tumour type.

Tumour DNA analysis has also identified 254 somatic NF1 gene lesions, including nonsense, missense, splice site, microdeletion/microinsertions $(<20 \mathrm{bp})$, indels (combined insertion-deletion events) and larger ( $>20 \mathrm{bp}$ ) deletions/insertions (Tables 3, S2). The consequences of all deletions and insertions for the reading frame were also determined, with five sequence changes being compound heterozygous NF1 mutations found in five haemopoietic tumours; however, with no other tissue available for analysis, it was not possible to differentiate between germline and somatic NF1 point mutations (Table S2). About 75 per cent
Table 2: Mechanistic basis of the NFI gene-associated LOH identified in different $\mathrm{NFI}$-associated tumours

\begin{tabular}{|c|c|c|}
\hline Tumour type & $\begin{array}{l}\text { Tumour showing } \\
\text { mitotic } \\
\text { recombination } \\
\text { (number \& } \\
\text { percentile) }\end{array}$ & $\begin{array}{l}\text { Tumours with } \\
\text { genomic } \\
\text { deletions } \\
\text { (number \& } \\
\text { percentile) }\end{array}$ \\
\hline Dermal neurofibroma & $28(76 \%)$ & $8(24 \%)$ \\
\hline $\begin{array}{l}\text { Plexiform } \\
\text { neurofibroma }\end{array}$ & II (44\%) & $14(56 \%)$ \\
\hline Spinal neurofibroma & 7 (88\%) & I (I2\%) \\
\hline MPNST & $5(16 \%)$ & $26(84 \%)$ \\
\hline Astrocytoma & $0(0 \%)$ & $2(100 \%)$ \\
\hline GIST/gastric carcinoid & I (50\%) & I (50\%) \\
\hline JMML & 15 (79\%) & $4(21 \%)$ \\
\hline Phaeochromocytoma & $0(0 \%)$ & $0(0 \%)$ \\
\hline Glomus tumour & I (100\%) & $0(0 \%)$ \\
\hline
\end{tabular}

Tabulated information only given for tumours in which the precise LOH mechanism was identifiable.

$(191 / 254)$ of the somatic mutations associated with NF1 tumours comprise mutations that are predicted to give rise to truncated proteins. Of these 191 changes, only 18 result from the insertion or duplication of bases; the remaining 173 truncations arise from deletion, nonsense mutation or frameshift events. Splice site mutations form a considerable proportion (39/254; 15.0 per cent) of the mutational spectrum, while missense changes only account for some 9.4 per cent $(24 / 254)$ of the somatic NF1 mutations.

Any attempt to make direct comparisons between the various tumour types would be unwise at this stage, owing to the paucity of somatic mutation data, especially for the less commonly encountered tumours. Table 3 nevertheless attempts to summarise the available data. The bias inherent in the data is immediately evident, with $211 / 254$ (83 per cent) mutational changes originating from the analysis of cutaneous neurofibroma DNA. Hence, the relative frequencies of the various mutation types in cutaneous neurofibromas are essentially comparable with the germline mutational spectrum, with nonsense mutations, splice site mutations and missense 
Table 3: The spectrum and percentile distribution of somatic NFI micro-lesions reported in different NFI-associated tumours

\begin{tabular}{|c|c|c|c|c|c|c|c|c|}
\hline \multirow[t]{2}{*}{ Tumour type } & \multicolumn{8}{|c|}{ Mutation type } \\
\hline & Deletion & Insertion & Indel & Nonsense & Splice site & Missense & Truncating & Total \\
\hline Dermal neurofibroma & $82(39 \%)$ & 15 (7\%) & $2(1 \%)$ & $59(28 \%)$ & $32(15 \%)$ & $21(10 \%)$ & I 58 (75\%) & 211 \\
\hline $\begin{array}{l}\text { Plexiform } \\
\text { neurofibroma }\end{array}$ & $6(33 \%)$ & I (6\%) & - & 7 (39\%) & $2(11 \%)$ & $2(11 \%)$ & 14 (78\%) & 18 \\
\hline Spinal neurofibroma & - & - & - & - & $2(66 \%)$ & I (33\%) & 0 & 3 \\
\hline MPNST & $7(70 \%)$ & I (10\%) & I (10\%) & I (10\%) & - & - & $10(100 \%)$ & 10 \\
\hline GIST/gastric carcinoid & I (20\%) & - & - & $3(60 \%)$ & I (20\%) & - & $4(80 \%)$ & 5 \\
\hline $\mathrm{JMML}^{*}$ & $*$ & $*$ & $*$ & I (100\%) & $*$ & $*$ & I (100\%) & I \\
\hline Glomus tumour & $2(33 \%)$ & I (I7\%) & - & I (I7\%) & $2(33 \%)$ & - & $4(67 \%)$ & 6 \\
\hline Overall & 98 (39\%) & $18(7 \%)$ & $3(1 \%)$ & $72(28 \%)$ & $39(15 \%)$ & $24(9 \%)$ & 191 (75\%) & 254 \\
\hline
\end{tabular}

alterations found in cutaneous neurofibromas at frequencies of 28 per cent (59/211), 15 per cent (32/ 211) and 10 per cent $(21 / 211)$, respectively (Table 3). Table 3 does, however, serve to highlight the high proportion of truncating mutations (191/ 254; 75 per cent) involved in the somatic inactivation of the NF1 gene in all tumour types, especially cutaneous neurofibromas.

An additional comparison between the frequency distributions of somatic microlesions and $\mathrm{LOH}$ is made in Table 1. There appears to be a marked difference between cutaneous neurofibromas, PNFs and MPNSTs, with 40 per cent, 79 per cent and 85 per cent, respectively, of somatic mutation events represented by $\mathrm{LOH}$. This may be explained in part by the extent of the molecular rearrangements in each tumour type; MPNSTs, for example, would be predicted to exhibit a greater extent of genetic aberration than a benign dermal neurofibroma. The types of analyses performed, however, will have a direct influence on such conclusions, in that either microlesions or LOH may not be screened for in some studies.

In summary, the more severe MPNSTs show a greater degree of genetic abnormality than other tumour types, with $\mathrm{LOH}$ constituting a much more frequent event in these tumours. Further comparison within and between the rarer tumour types would not be valid, however, owing to the relative paucity of mutation data currently available for analysis.

\section{Mutational mechanisms underlying the known somatic NFI gene lesions}

Somatic inactivation of the NF1 gene may result from different mutational mechanisms and may involve intragenic mutations, $\mathrm{LOH}$ and epigenetic modification of the promoter region. Among the 254 somatic NF1 mutations listed in Table S2, 72 nonsense mutations were found, of which 36 involved mutations in just 15 codons in different tumours (codons 192, 304, 426, 440, 816, 1241, 1306, 1362, 1513, 1569, 1604, 1748, 1939, 1976 and 2429), with many previously reported in different tumours or different studies. Ten of these 15 different recurrent nonsense mutations involve $\mathrm{C}>$ $\mathrm{T}$ or $\mathrm{G}>\mathrm{A}$ transitions within $\mathrm{CpG}$ dinucleotides and are compatible with the endogenous mutational mechanism of methylation-mediated deamination of 5-methylcytosine $(5 \mathrm{mC})$. Of these 72 nonsense mutations, 28 have also been reported as germline mutations in NF1 patients (Human Gene Mutation Database [HGMD]), ${ }^{81}$ indicating that the same mutational mechanism is operating in both the soma and 
germline. The importance of this mutational mechanism is evidenced by the finding that 12 of the 15 recurrent somatic nonsense mutations have also been reported independently in the germline (codons 192, 304, 426, 440, 816, 1241, 1306, 1362, 1513, 1569, 1748 and 2429). For the ten of these 15 nonsense mutations that correspond to $\mathrm{C}>\mathrm{T}$ or $\mathrm{G}>\mathrm{A}$ transitions within $\mathrm{CpG}$ dinucleotides, we may infer that the mutated cytosine must be methylated both in the soma and in the germline, thereby explaining the vulnerability of these sites to methylation-mediated deamination in both cell lineages.

Among the somatic NF1 mutations listed in Table S2 are 21 different missense mutations. Of these, two (in codons 519 and 776) have been reported more than once in different tumours or different studies, although neither is compatible with methylation-mediated deamination of $5 \mathrm{mC}$. Of the 21 missense mutations, only one (in codon 176) has also been reported in the germline (see HGMD). Since this Asp176Glu mutation has also been reported more than once in NF1-associated tumours, it may well be that this residue is of importance for the function of neurofibromin in both the soma and the germline. Furthermore, this residue is conserved in different species, including Drosophila and Fugu, and has not been identified in 250 unrelated normal individuals.

Nonsense mutations are not the only type of NF1 mutation to occur recurrently in the soma. Among the somatic NF1 microdeletions listed in Table S2 are five that have been reported more than once in different tumours (c.1888delG, c.2033delC, c.3058delG, c.4374_4375delCC and c.5731delT) with three microdeletions occurring in mononucleotide tracts $\left(G_{4}, C_{7}\right.$ and $T_{3}$, respectively), suggestive of a model of slipped mispairing at the DNA replication fork. Importantly, c.2033delC has also been reported in the germline (see HGMD), indicating that this tetranucleotide stretch is a hotspot for mutation in both the germline and the soma. A microinsertion (c.1733insT, located within a $\mathrm{T}_{6}$ tract) has also been found to occur recurrently in the soma but this has not so far been reported in the germline. The reader interested in a detailed comparative analysis of germline and somatic mutations in human TSGs is referred to Ivanov et al. ${ }^{82}$

\section{NFI gene somatic mutations in non-NF I -associated tumours}

Various studies have identified somatic NF1 gene mutations in non-NF1-associated cancers. Thus, somatic NF1 aberrations have been identified in glioblastoma multiforme (GBM) tumours, lung adenocarcinomas, malignant breast tumours, leukaemia, ovarian serous carcinomas (OSCs) and neuroblastoma. ${ }^{10-12,14-16,83}$ Some of the NF1 gene changes are relatively frequent in these tumours and therefore have the potential to represent specific prognostic and diagnostic markers. For example, 23 per cent of sporadic GBM tumours harbour an inactivating NF1 somatic mutation, and this may enable such GBM tumours to differentiate into the mesenchymal molecular subclass. ${ }^{13}$ Similarly, in 22 per cent $(9 / 41)$ of primary OSCs, an NF1 mutation was detected, six of which exhibited biallelic inactivation. ${ }^{12}$ Interestingly, all nine of these OSC samples also contained a TP53 mutation, highlighting the likely involvement of this TSG in OSC pathogenesis. ${ }^{12}$

Given the pivotal role that neurofibromin plays in several cell signalling pathways, it is not surprising that its loss will affect distinct molecular subtypes in different cancers. Indeed, the efficacy of any future therapeutic intervention for many tumours will almost certainly hinge upon our ability successfully to identify such molecular subclasses of tumour.

\section{Prospects for the development of new treatments/therapies}

As the complex picture underlying the molecular nature of NF1 tumorigenesis becomes better defined, the treatment regimens available to patients should greatly improve. Although the future is encouraging, the optimal treatment for NF1 tumours currently rests with their surgical resection, in spite of the high chance of recurrent malignancy. Gottfried and colleagues ${ }^{84}$ have suggested that the recruitment of supporting cells around the 
neurofibroma, coupled with aberrant Remak bundles, could explain how the neurofibroma integrates into the surrounding tissue, and it is this that may lead to the surgical difficulties that often lead to tumour recurrence. Moreover, it has been suggested that surgical interference may even increase the recruitment of surrounding cell types, thereby inadvertently increasing the growth of lesions leading to the formation of new neurofibromas. ${ }^{84}$ Surgical biopsy is therefore inherently problematic, and novel therapeutics are urgently required. Clinical and preclinical trials targeting different components of the Ras/MAPK signalling pathway and related growth factor receptors appear to be more promising. It is likely, however, that treatment with multiple drugs may be more effective for NF1 tumours. ${ }^{5}$

\section{Concluding remarks}

Biallelic inactivation of the NF1 gene, resulting in the complete loss of functional neurofibromin, initiates the pathogenic process that eventually results in the formation of nerve sheath tumours. NF1 gene inactivation may occur through relatively subtle lesions that affect just a few DNA bases, or may involve large genomic changes that affect large chromosomal regions, or even the entire chromosome 17. This review demonstrates that NF1-associated tumour types display a considerable degree of variation in terms of the level of $\mathrm{LOH}$ detected, with cutaneous neurofibromas, PNFs and MPNSTs. MPNSTs manifest increased levels of deletion-based LOH, whereas cutaneous neurofibromas appear to be associated with a localised deletion of the NF1 gene through mitotic recombination (the situation in PNFs being somewhat intermediate). In MPNSTs, additional mutations at different gene loci are almost certainly involved in the progression of the tumour.

In terms of the molecular mechanisms of mutagenesis, both methylation-mediated deamination of 5-methylcytosine and slipped mispairing within polynucleotide tracts appear to be responsible for the occurrence of mutation hotspots in both the germline and the soma. For some types of tumour, there is interplay between the soma and the germline, in that the location of the germline mutation can influence the nature, frequency and location of the subsequent somatic mutation. ${ }^{85,86}$ As yet, however, there is no evidence for this phenomenon in the context of NF1 tumorigenesis.

Although our knowledge of the role of the NF1 gene in tumorigenesis is ever expanding, definitive markers of malignant transformation remain to be discovered. Mouse and other animal models, including zebrafish, ${ }^{87}$ have provided new perspectives for research, with various knockout and mutagenesis studies potentiating functional studies. It is already clear that, in order to clarify the role of the NF1 gene in NF1-associated tumours, we must improve our understanding of the significance of the somatic (second-hit) mutations. The brief assessment of the compilation of somatic NF1 mutations in NF1-associated tumour types reported here failed to unearth any specific genotypic correlations. The limited size of the mutation dataset means that reliable conclusions are hard to draw, and that larger and better-defined patient groups will be needed, to allow more reliable comparisons to be made. Additionally, definitive prognostic markers should be identified that permit differentiation between benign neurofibromas that are likely to progress to malignancy and those that are not.

This review nevertheless emphasises that NF1 is a highly individual condition that exhibits extreme somatic mutational heterogeneity both within and between patients. These are the mutations which are ultimately responsible for the molecular changes that can lead to tumour formation. If we can come to understand how these changes bring about tumorigenesis, we shall be better placed not only with respect to the provision of genetic counselling, but also in terms of exploring new avenues for the development of new drug-based therapies.

\section{Acknowledgments}

We are grateful to all our NF1 patients and their families for their support. We also thank Laura Thomas and Gill Spurlock for their help with the compilation of mutation data. 


\section{References}

1. Huson, S., Harper, P. and Compston, D. (1988), 'Von Recklinghausen neurofibromatosis. A clinical and population study in south-east Wales', Brain Vol. 111, pp. 1355-1381.

2. Lammert, M., Friedman, J.M., Kluwe, L. and Mautner, V.F. (2005), 'Prevalence of neurofibromatosis 1 in German children at elementary school enrollment', Arch. Dermatol. Vol. 141, pp. 71-74.

3. Upadhyaya, M., Huson, S., Davies, M., Thomas, N. et al. (2007), 'An absence of cutaneous neurofibromas associated with a 3-bp inframe deletion in exon 17 of the NF1 gene (c.2970-2972 delAAT): Evidence of a clinically significant NF1 genotype-phenotype correlation', Am. J. Hum. Genet. Vol. 80, pp. 140-151.

4. Upadhyaya, M. (2011), 'Genetic basis of tumorigenesis in NF1 malignant peripheral nerve sheath tumors', Front. Biosci. Vol. 16, pp. 937-951.

5. Upadhyaya, M. (2010), 'Neurofibromatosis type 1 (NF1): Diagnosis and recent advances', Expert Opin. Med. Genet. Vol. 4, pp. 307-322.

6. Knudson, A. J. (1971), 'Mutation and cancer: Statistical study of retinoblastoma', Proc. Natl. Acad. Sci. USA Vol. 68, pp. 820-823.

7. Pao, W. and Girard, N. (2011), 'New driver mutations in non-small-cell lung cancer', Lancet Oncol. Vol. 12, pp. 175-180.

8. Sawada, S., Florell, S., Purandare, S., Ota, M. et al. (1996), 'Identification of NF1 mutations in both alleles of a dermal neurofibroma', Nat. Genet. Vol. 14, pp. 110-112.

9. Serra, E., Puig, S., Otero, D., Gaona, A. et al. (1997), 'Confirmation of a double-hit model for the NF1 gene in benign neurofibromas', Am. J. Hum. Genet. Vol. 61, pp. 512-519.

10. Parsons, D.W., Jones, S., Zhang, X., Lin, J.C. et al. (2008), 'An integrated genomic analysis of human glioblastoma multiforme', Science Vol. 321, pp. $1807-1812$.

11. Ding, L., Getz, G., Wheeler, D., Mardis, E. et al. (2008), 'Somatic mutations affect key pathways in lung adenocarcinoma', Nature Vol. 455 , pp. 1069-1075.

12. Sangha, N., Wu, R., Kuick, R., Powers, S. et al. (2008), 'Neurofibromin 1 (NF1) defects are common in human ovarian serous carcinomas and co-occur with TP53 mutations', Neoplasia Vol. 10, pp. 1362-1372.

13. Brennan, C., Momota, H., Hambardzumyan, D., Ozawa, T. et al. (2009), 'Glioblastoma subclasses can be defined by activity among signal transduction pathways and associated genomic alterations', PLoS One Vol. 4, p. e7752.

14. McGillicuddy, L.T., Fromm, J.A., Hollstein, P.E., Kubek, S. et al. (2009), 'Proteasomal and genetic inactivation of the NF1 tumor suppressor in gliomagenesis', Cancer Cell Vol. 16, pp. 44- 54.

15. Haferlach, C., Dicker, F, Kohlmann, A., Schindela, S. et al. (2010), 'AML with CBFB-MYH11 rearrangement demonstrate RAS pathway alterations in $92 \%$ of all cases including a high frequency of NF1 deletions', Lenkemia Vol. 24, pp. 1065-1069.

16. Hölzel, M., Huang, S., Koster, J., Ora, I. et al. (2010), 'NF1 is a tumor suppressor in neuroblastoma that determines retinoic acid response and disease outcome', Cell Vol. 142, pp. 218-229.

17. Easton, D.F., Ponder, M.A., Huson, S.M. and Ponder, B.A. (2008), 'An analysis of variation in expression of neurofibromatosis (NF) type 1 (NF1): Evidence for modifying genes', Am. J. Hum. Genet. Vol 53 , pp. 305-313.

18. Kehrer-Sawatzki, H. and Cooper, D.N. (2008), 'Mosaicism in sporadic neurofibromatosis type 1: Variations on a theme common to other hereditary cancer syndromes?', J. Med. Genet. Vol. 45, pp. 622-631.

19. Ballester, R., Marchuk, D., Boguski, M., Saulino, A. et al. (1990), 'The NF1 locus encodes a protein functionally related to mammalian GAP and yeast IRA proteins', Cell Vol. 63, pp. 851-859.

20. Upadhyaya, M., Kluwe, L., Spurlock, G., Monem, B. et al. (2008), 'Germline and somatic NF1 gene mutation spectrum in NF1-associated malignant peripheral nerve sheath tumors (MPNSTs)', Hum. Mutat. Vol. 29 , pp. $74-82$.

21. Andersen, L., Ballester, R., Marchuk, D., Chang, E. et al. (1993), 'A conserved alternative splice in the von Recklinghausen neurofibromatosis (NF1) gene produces two neurofibromin isoforms, both of which have GTPase-activating protein activity', Mol. Cell. Biol. Vol. 13, pp. $487-495$.

22. Danglot, G., Régnier, V., Fauvet, D., Vassal, G. et al. (1995), 'Neurofibromatosis 1 (NF1) mRNAs expressed in the central nervous system are differentially spliced in the 5' part of the gene', Hum. Mol. Genet. Vol. 4, pp. 915-920.

23. Kaufmann, D., Müller, R., Kenner, O., Leistner, W. et al. (2002), 'The N-terminal splice product NF1-10a-2 of the NF1 gene codes for a transmembrane segment', Biochem. Biophys. Res. Commun. Vol. 294, pp. 496-503.

24. Gutmann, D., Geist, R., Rose, K. and Wright, D. (1995), 'Expression of two new protein isoforms of the neurofibromatosis type 1 gene product, neurofibromin, in muscle tissues', Dev. Dyn. Vol. 202, pp. 302-311.

25. Upadhyaya, M. (2008), 'NF1 gene structure and NF1 genotype/phenotype correlations', In: Kaufmann, D. (ed.), Neurofibromatoses, Karger, Basel, pp. 46-62.

26. Bennett, E., Thomas, N. and Upadhyaya, M. (2009), 'Neurofibromatosis type 1: Its association with the Ras/MAPK pathway syndromes', J. Paediatr. Neurol. Vol. 7, pp. 105-115.

27. Cichowski, K., Shih, T., Schmitt, E., Santiago, S. et al. (1999), 'Mouse models of tumor development in neurofibromatosis type 1', Science Vol. 286, pp. 2172-2176.

28. Cichowski, K. and Jacks, T. (2001), 'NF1 tumor suppressor gene function: Narrowing the GAP', Cell Vol. 104, pp. 593-604.

29. Serra, E., Rosenbaum, T., Winner, U., Aledo, R. et al. (2000), 'Schwann cells harbor the somatic NF1 mutation in neurofibromas: Evidence of two different Schwann cell subpopulations', Hum. Mol. Genet. Vol. 9, pp. 3055-3064.

30. Le, L., Shipman, T., Burns, D. and Parada, L. (2009), 'Cell of origin and microenvironment contribution for NF1-associated dermal neurofibromas', Cell Stem Cell Vol. 4, pp. 453-463.

31. McLaughlin, M.E. and Jacks, T. (2003), 'Progesterone receptor expression in neurofibromas', Cancer Res. Vol. 63, pp. 752-755.

32. Dugoff, L. and Sujansky, E. (1996), 'Neurofibromatosis type 1 and pregnancy', Am. J. Med. Genet. Vol. 66, pp. 7-10.

33. Roth, T., Ramamurthy, P., Muir, D., Wallace, M. et al. (2008), 'Influence of hormones and hormone metabolites on the growth of Schwann cells derived from embryonic stem cells and on tumor cell lines expressing variable levels of neurofibromin', Dev. Dyn. Vol. 237, pp. 513-524.

34. Wu, J., Williams, J., Rizvi, T., Kordich, J. et al. (2008), 'Plexiform and dermal neurofibromas and pigmentation are caused by Nf1 loss in desert hedgehog-expressing cells', Cancer Cell Vol. 13, pp. 105-116.

35. Joseph, N., Mosher, J., Buchstaller, J., Snider, P. et al. (2008), 'The loss of Nf1 transiently promotes self-renewal but not tumorigenesis by neural crest stem cells', Cancer Cell Vol. 13, pp. 129-140.

36. Parrinello, S., Noon, L., Harrisingh, M., Digby, P. et al. (2008), 'NF1 loss disrupts Schwann cell-axonal interactions: A novel role for semaphorin 4F', Genes Dev. Vol. 22, pp. 3335-3348.

37. Zhu, Y., Ghosh, P., Charnay, P., Burns, D. et al. (2002), 'Neurofibromas in NF1: Schwann cell origin and role of tumor environment', Science Vol. 296, pp. 920-922.

38. Ingram, D., Yang, F., Travers, J., Wenning, M. et al. (2000), 'Genetic and biochemical evidence that haploinsufficiency of the Nf1 tumor suppressor gene modulates melanocyte and mast cell fates in vivo', J. Exp. Med. Vol. 191, pp. 181-188.

39. Yang, F.C., Ingram, D.A., Chen, S., Zhu, Y. et al. (2008), 'Nf1-dependent tumors require a microenvironment containing $\mathrm{Nf1}+/-$ and $\mathrm{c}_{-}$ kit-dependent bone marrow', Cell Vol. 135, pp. 437-448.

40. Serra, E., Rosenbaum, T., Nadal, M., Winner, U. et al. (2001), 'Mitotic recombination effects homozygosity for NF1 germline mutations in neurofibromas', Nat. Genet. Vol. 28, pp. 294-296.

41. Thomas, L., Kluwe, L., Chuzhanova, N., Mautner, V. et al. (2010), 'Analysis of NF1 somatic mutations in cutaneous neurofibromas from patients with high tumor burden', Neurogenetics Vol. 11, pp. 391-400.

42. Garcia-Linares, C., Fernandez-Rodriguez, J., Terribas, E., Mercade, J. et al. (2011), 'Dissecting loss of heterozygosity (LOH) in neurofibromatosis type 1-associated neurofibromas: Importance of copy neutral LOH', Hum. Mutat. Vol. 32, pp. 78-90. 
43. Ducatman, B., Scheithauer, B., Piepgras, D., Reiman, H. et al. (1986), 'Malignant peripheral nerve sheath tumors. A clinicopathologic study of 120 cases', Cancer Vol. 57, pp. 2006-2021.

44. Evans, D., Baser, M., McGaughran, J., Sharif, S. et al. (2002), 'Malignant peripheral nerve sheath tumours in neurofibromatosis 1', J. Med. Genet. Vol. 39, pp. 311-314.

45. McCaughan, J., Holloway, S., Davidson, R. and Lam, W. (2007), 'Further evidence of the increased risk for malignant peripheral nerve sheath tumour from a Scottish cohort of patients with neurofibromatosis type 1', J. Med. Genet. Vol. 44, pp. 463-466.

46. Spurlock, G., Griffiths, S., Uff, J. and Upadhyaya, M. (2007), 'Somatic alterations of the NF1 gene in an NF1 individual with multiple benign tumours (internal and external) and malignant tumour types', Fam. Cancer Vol. 6, pp. 463-471.

47. Benz, M.R., Czernin, J., Dry, S.M., Tap, W.D. et al. (2010), 'Quantitative F18-fluorodeoxyglucose positron emission tomography accurately characterizes peripheral nerve sheath tumors as malignant or benign', Cancer Vol. 116, pp. 451-458.

48. De Raedt, T., Brems, H., Wolkenstein, P., Vidaud, D. et al. (2003), 'Elevated risk for MPNST in NF1 microdeletion patients', Am. J. Hum. Genet. Vol. 72, pp. 1288-1292.

49. Upadhyaya, M., Spurlock, G., Majounie, E., Griffiths, S. et al. (2006), 'The heterogeneous nature of germline mutations in NF1 patients with malignant peripheral serve sheath tumours (MPNSTs)', Hum. Mutat. Vol. 27, pp. 716.

50. Mautner, V.F., Kluwe, L., Friedrich, R.E., Roehl, A.C. et al. (2010), 'Clinical characterisation of 29 neurofibromatosis type-1 patients with molecularly ascertained 1.4 Mb type-1 NF1 deletions', J. Med. Genet. Vol. 47, pp. 623-630.

51. Pasmant, E., Vidaud, D., Harrison, M. and Upadhyaya, M. (2010), 'Different sized somatic NF1 locus rearrangements in neurofibromatosis 1-associated malignant peripheral nerve sheath tumors', J. Neurooncol. Vol. 102, pp. 341-346.

52. Legius, E., Dierick, H., Wu, R., Hall, B. et al. (1994), 'TP53 mutations are frequent in malignant NF1 tumors', Genes Chromosomes Cancer Vol. 10 , pp. $250-255$

53. Menon, A., Anderson, K., Riccardi, V., Chung, R. et al. (1990), 'Chromosome $17 \mathrm{p}$ deletions and p53 gene mutations associated with the formation of malignant neurofibrosarcomas in von Recklinghausen neurofibromatosis', Proc. Natl. Acad. Sci. USA Vol. 87, pp. 5435-5439.

54. Vogel, K., Klesse, L., Velasco-Miguel, S., Meyers, K. et al. (1999), 'Mouse tumor model for neurofibromatosis type 1', Science Vol. 286, pp. $2176-2179$

55. Kourea, H., Orlow, I., Scheithauer, B., Cordon-Cardo, C. et al. (1999), 'Deletions of the INK4A gene occur in malignant peripheral nerve sheath tumors but not in neurofibromas', Am. J. Pathol. Vol. 155, pp. 1855-1860.

56. Mantripragada, K., Spurlock, G., Kluwe, L., Chuzhanova, N. et al. (2008), 'High-resolution DNA copy number profiling of malignant peripheral nerve sheath tumors using targeted microarray-based comparative genomic hybridization', Clin. Cancer Res. Vol. 14, pp. 1015-1024.

57. Nielsen, G., Stemmer-Rachamimov, A., Ino, Y., Moller, M. et al. (1999), 'Malignant transformation of neurofibromas in neurofibromatosis 1 is associated with CDKN2A/p16 inactivation', Am. J. Pathol. Vol. 155, pp. 1879-1884

58. Gregorian, C., Nakashima, J., Dry, S., Nghiemphu, P. et al. (2009), 'PTEN dosage is essential for neurofibroma development and malignant transformation', Proc. Natl. Acad. Sci. USA Vol. 106, pp. 19479-19484.

59. Subramanian, S., Thayanithy, V., West, R., Lee, C. et al. (2010), 'Genome-wide transcriptome analyses reveal p53 inactivation mediated loss of miR-34a expression in malignant peripheral nerve sheath tumours', J. Pathol. Vol. 220, pp. 58-70.

60. Chai, G., Liu, N., Ma, J., Li, H. et al. (2010), 'MicroRNA-10b regulates tumorigenesis in neurofibromatosis type 1', Cancer Sci. Vol. 101, pp. 1997-2004.

61. Ars, E., Kruyer, H., Gaona, A., Casquero, P. et al. (1998), 'A clinical variant of neurofibromatosis type 1: Familial spinal neurofibromatosis with a frameshift mutation in the NF1 gene', Am. J. Hum. Genet. Vol. 62, pp. 834-841.

62. Poyhonen, M., Leisti, E., Kytölä, S. and Leisti, J. (1997), 'Hereditary spinal neurofibromatosis: A rare form of NF1?', J. Med. Genet. Vol. 34, pp. $184-187$.

63. Pulst, S.M., Riccardi, V.M., Fain, P. and Korenberg, J.R. (1991), 'Familial spinal neurofibromatosis: Clinical and DNA linkage analysis', Neurology Vol. 41, pp. 1923-1927.

64. Upadhyaya, M., Spurlock, G., Kluwe, L., Chuzhanova, N. et al. (2009), 'The spectrum of somatic and germline NF1 mutations in NF1 patients with spinal neurofibromas', Neurogenetics Vol. 10, pp. 251-263.

65. Miettinen, M., Fetsch, J., Sobin, L. and Lasota, J. (2006), 'Gastrointestinal stromal tumors in patients with neurofibromatosis 1: A clinicopathologic and molecular genetic study of 45 cases', Am. J. Surg. Pathol. Vol. 30, pp. 90-96.

66. Maertens, O., Prenen, H., Debiec-Rychter, M., Wozniak, A. et al. (2006), 'Molecular pathogenesis of multiple gastrointestinal stromal tumors in NF1 patients', Hum. Mol. Genet. Vol. 15, pp. 1015-1023.

67. Stewart, W., Traynor, J.P., Cooke, A., Griffiths, S. et al. (2007), 'Gastric carcinoid: Germline and somatic mutation of the neurofibromatosis type 1 gene', Fam. Cancer, Vol. 6, pp. 147-152.

68. Stiller, C., Chessells, J. and Fitchett, M. (1994), 'Neurofibromatosis and childhood leukaemia/lymphoma: a population-based UKCCSG study', Br. J. Cancer Vol. 70, pp. 969-972.

69. Flotho, C., Steinemann, D., Mullighan, C., Neale, G. et al. (2007), 'Genome-wide single-nucleotide polymorphism analysis in juvenile myelomonocytic leukemia identifies uniparental disomy surrounding the NF1 locus in cases associated with neurofibromatosis but not in cases with mutant RAS or PTPN11', Oncogene Vol. 26, pp. 5816-5821.

70. Yoshimi, A., Kojima, S. and Hirano, N. (2010), 'Juvenile myelomonocytic leukemia: Epidemiology, etiopathogenesis, diagnosis, and management considerations', Paediatr. Drugs Vol. 12, pp. 11-21.

71. Sugimoto, Y., Muramatsu, H., Makishima, H., Prince, C. et al. (2009), 'Spectrum of molecular defects in juvenile myelomonocytic leukaemia includes ASXL1 mutations', Br. J. Haematol. Vol. 150, pp. 83-87.

72. Steinemann, D., Arning, L., Praulich, I., Stuhrmann, M. et al. (2010), 'Mitotic recombination and compound-heterozygous mutations are predominant NF1-inactivating mechanisms in children with juvenile myelomonocytic leukemia and neurofibromatosis type 1', Haematologica Vol. 95, pp. 320-323

73. Chen, J.M., Cooper, D.N., Ferec, C., Kehrer-Sawatzki, H. et al. (2010), 'Genomic rearrangements in inherited disease and cancer', Semin. Cancer Biol. Vol. 20, pp. 222-233.

74. Stephens, K., Weaver, M., Leppig, K., Maruyama, K. et al. (2006), 'Interstitial uniparental isodisomy at clustered breakpoint intervals is a frequent mechanism of NF1 inactivation in myeloid malignancies', Blood Vol. 108, pp. 1684-1689.

75. Listernick, R., Charrow, J., Greenwald, M. and Mets, M. (1994), 'Natural history of optic pathway tumors in children with neurofibromatosis type 1: A longitudinal study', J. Pediatr. Vol. 125, pp. 63-66.

76. Gutmann, D., James, C., Poyhonen, M., Louis, D. et al. (2003), 'Molecular analysis of astrocytomas presenting after age 10 in individuals with NF1', Neurology Vol. 61, pp. 1397-1400.

77. Gutmann, D., Donahoe, J., Brown, T., James, C. et al. (2000), 'Loss of neurofibromatosis 1 (NF1) gene expression in NF1-associated pilocytic astrocytomas', Neuropathol. Appl. Neurobiol. Vol. 26, pp. $361-367$.

78. Bausch, B., Borozdin, W., Mautner, V.F., Hoffmann, M.M. et al. (2007), 'Germline NF1 mutational spectra and loss-of-heterozygosity analyses in patients with pheochromocytoma and neurofibromatosis type 1', J. Clin. Endocrinol. Metab. Vol. 92, pp. 2784-2792.

79. Petri, B.J., van Eijck, C.H., de Herder, W.W., Wagner, A. et al. (2009), 'Phaeochromocytomas and sympathetic paragangliomas', $\mathrm{Br}$. J. Surg. Vol. 96, pp. 1381-1392.

80. Brems, H., Park, C., Maertens, O., Pemov, A. et al. (2009), 'Glomus tumors in neurofibromatosis type 1: Genetic, functional, and clinical evidence of a novel association', Cancer Res. Vol. 69, pp. 7393-7401. 
81. Stenson, P.D., Ball, E., Howells, K., Phillips, A. et al. (2008), 'Human Gene Mutation Database: Towards a comprehensive central mutation database', J. Med. Genet. Vol. 45, pp. 124-126.

82. Ivanov, D., Hamby, S.E., Stenson, P.D., Phillips, A.D. et al. (2011), 'Comparative analysis of germline and somatic microlesion mutational spectra in 17 human tumor suppressor genes', Hum. Mutat. Vol. 32, pp. 620-632.

83. Lee, J., Wang, J., Torbenson, M., Lu, Y. et al. (2010), 'Loss of SDHB and NF1 genes in a malignant phyllodes tumor of the breast as detected by oligo-array comparative genomic hybridization', Cancer Genet. Cytogenet Vol. 196, pp. 179-183.

84. Gottfried, O., Viskochil, D., Fults, D. and Couldwell, W. (2006), 'Molecular, genetic, and cellular pathogenesis of neurofibromas and surgical implications', Neurosurgery Vol. 58, pp. 1-16; discussion $11-16$

85. Lamlum, H., Ilyas, M., Rowan, A., Clark, S. et al. (1999), 'The type of somatic mutation at APC in familial adenomatous polyposis is determined by the site of the germline mutation: A new facet to Knudson's 'two-hit' hypothesis', Nat. Med. Vol. 5, pp. 1071-1075.

86. Dworkin, A.M., Ridd, K., Bautista, D., Allain, D.C. et al. (2010), 'Germline variation controls the architecture of somatic alterations in tumors', PLoS Genet. Vol. 6, p. e1001136.

87. Padmanabhan, A., Lee, J., Ismat, F., Lu, M. et al. (2009), 'Cardiac and vascular functions of the zebrafish orthologues of the type I neurofibromatosis gene NFI', Proc. Natl. Acad. Sci. USA Vol. 106, pp. 22305-22310. 
Table SI. Summary of germline mutations and loss of heterozygosity (LOH) in NFI-associated tumours

\begin{tabular}{|c|c|c|c|c|c|c|c|c|c|}
\hline $\begin{array}{l}\text { Patient } \\
\text { ID }\end{array}$ & Germline mutation & $\begin{array}{l}\text { Type of } \\
\text { germline } \\
\text { mutation }\end{array}$ & LOH & LOH markers & $\begin{array}{l}\text { Predicted } \\
\text { extent of } \\
\text { LOH }\end{array}$ & $\begin{array}{l}\text { Evidence } \\
\text { for genomic } \\
\text { deletion? } \\
\text { MLPA/CGH } \\
\text { arrayCGH/ } \\
\text { FISH }\end{array}$ & $\begin{array}{l}\text { Probable } \\
\text { mechanism }\end{array}$ & $\begin{array}{l}\text { No. } \\
\text { samples } \\
\text { with } \\
\text { LOH }\end{array}$ & Reference \\
\hline \multicolumn{10}{|c|}{ Dermal neurofibromas } \\
\hline TI90.2 & Exon 2 and 3 deletion & $\begin{array}{l}\text { Two exon } \\
\text { deletion }\end{array}$ & Yes & $\begin{array}{l}\text { E5 RFLP, II } 2 b, \\
\text { IVS27A28.4, JI/J2, } \\
\text { EVI20, IVS38GT53.0, } \\
3^{\prime} \mathrm{NFI}, \text { C7CTI/2 } \\
\text { (3'UTR), EW206, } \\
\text { EW207, DI7S798, } \\
\text { DI7SI868 }\end{array}$ & $\begin{array}{l}\mathrm{NFI} \text { and } 3^{\prime} \\
\text { flanking } \\
\text { region }\end{array}$ & MLPA & Deletion & $5 / 23$ & I \\
\hline TI90.6 & & & Yes & $\begin{array}{l}\text { E5 RFLP, II } 2 \mathrm{~b}, \\
\text { IVS27A28.4, JI/J2, } \\
\text { EVI20, IVS38GT53.0, } \\
3^{\prime} \mathrm{NFI}, \mathrm{C} 7 \mathrm{CTI} / 2 \\
\text { (3'UTR), EW206 }\end{array}$ & $\begin{array}{l}\mathrm{NFI} \text { and } 3^{\prime} \\
\text { flanking } \\
\text { region }\end{array}$ & MLPA & Deletion & & \\
\hline TI90.II & & & Yes & $\begin{array}{l}\text { E5 RFLP, II } 2 \mathrm{~b}, \\
\text { IVS27A28.4, JI/J2, } \\
\text { EVI20, IVS38GT53.0, } \\
3^{\prime} \mathrm{NFI}, \mathrm{C} 7 \mathrm{CTI} / 2 \\
\left(3^{\prime} \text { UTR) }\right.\end{array}$ & $\begin{array}{l}\mathrm{NFI} \text { and } 3^{\prime} \\
\text { flanking } \\
\text { region }\end{array}$ & MLPA & Deletion & & \\
\hline TI90.17 & & & Yes & $\begin{array}{l}\text { E5 RFLP, II 2b, } \\
\text { IVS27A28.4, JI/J2 }\end{array}$ & $\begin{array}{l}\text { Intragenic } \\
\mathrm{NFI}\end{array}$ & MLPA & Deletion & & \\
\hline TI90.I8ii & & & Yes & E5 RFLP & $\begin{array}{l}\text { Intragenic } \\
\mathrm{NFI}\end{array}$ & MLPA & Deletion & & \\
\hline T206.I & $\begin{array}{l}\text { Ex4b: } \\
\text { c.499_502delTGTT; } \\
\text { p.CI67GnfsX9 }\end{array}$ & $\begin{array}{l}4 \text { bp } \\
\text { deletion } \\
\text { (FS) }\end{array}$ & & $\mathrm{LOH}$ & & NIA & & & $\begin{array}{l}\text { Unpublishec } \\
\text { data, Cardiff }\end{array}$ \\
\hline T206.2 & & & & $\mathrm{LOH}$ & & & & & \\
\hline T206.3 & & & & $\mathrm{LOH}$ & & & & & \\
\hline
\end{tabular}


Table SI. Continued

\begin{tabular}{|c|c|c|c|c|c|c|c|c|c|}
\hline $\begin{array}{l}\text { Patient } \\
\text { ID }\end{array}$ & Germline mutation & $\begin{array}{l}\text { Type of } \\
\text { germline } \\
\text { mutation }\end{array}$ & LOH & LOH markers & $\begin{array}{l}\text { Predicted } \\
\text { extent of } \\
\text { LOH }\end{array}$ & $\begin{array}{l}\text { Evidence } \\
\text { for genomic } \\
\text { deletion? } \\
\text { MLPA/CGH } \\
\text { arrayCGH/ } \\
\text { FISH }\end{array}$ & $\begin{array}{l}\text { Probable } \\
\text { mechanism }\end{array}$ & $\begin{array}{l}\text { No. } \\
\text { samples } \\
\text { with } \\
\text { LOH }\end{array}$ & Reference \\
\hline L002_3 & $\begin{array}{l}\text { Ex9: c. } 1246 \mathrm{C}>\mathrm{T} ; \\
\text { p.Arg416X }\end{array}$ & Nonsense & Yes & $\begin{array}{l}\text { rs } 29001484, \text { rs } 4583306, \\
\text { NFI germline mutation, } \\
\text { rs205509I, rs I I } 869264\end{array}$ & NFI & Array CGH & Deletion & $6 / 28$ & 2 \\
\hline L002_5 & & & Yes & $\begin{array}{l}\text { rs } 2900 \text { I } 484, \text { rs } 4583306, \\
\text { NFI germline mutation, } \\
\text { rs205509I, rs I I } 869264\end{array}$ & NFI & Array CGH & $\begin{array}{l}\text { Mitotic } \\
\text { recombination }\end{array}$ & & \\
\hline L002_I & & & Yes & $\begin{array}{l}\text { rs } 29001484, \text { rs } 4583306, \\
\text { NFI germline mutation, } \\
\text { rs205509I, rs I I } 869264\end{array}$ & NFI & Array CGH & $\begin{array}{l}\text { Mitotic } \\
\text { recombination }\end{array}$ & & \\
\hline L002 C & & & Yes & NS & & NIA & & $3 / 38$ & 3 \\
\hline T473.IA & $\begin{array}{l}\text { Ex|0b: c.|4|3- } \\
\text { I4|4delAG; } \\
\text { p.Lys47|AsnfsX4 }\end{array}$ & $\begin{array}{l}2 \text { bp } \\
\text { deletion } \\
\text { (FS) }\end{array}$ & Yes & $\begin{array}{l}\mathrm{HHH} 202, \text { JIJ2, IVS27, } \\
\text { EVI20, IVS38 }\end{array}$ & NFI & MLPA & $\begin{array}{l}\text { Mitotic } \\
\text { recombination }\end{array}$ & $22 / 89$ & 4 \\
\hline T473.IC & & & Yes & $\mathrm{HHH} 202, \mathrm{JIJ} 2, \mathrm{EV} 120$ & NFI & MLPA & $\begin{array}{l}\text { Mitotic } \\
\text { recombination }\end{array}$ & & \\
\hline T473.3 & & & Yes & JIJ2, EVI20, IVS38 & NFI & MLPA & $\begin{array}{l}\text { Mitotic } \\
\text { recombination }\end{array}$ & & \\
\hline T473.5 & & & Yes & $\begin{array}{l}\mathrm{HHH} 202, \mathrm{JIJ} 2, \mathrm{EVI} 20, \\
\text { IVS38 }\end{array}$ & NFI & MLPA & $\begin{array}{l}\text { Mitotic } \\
\text { recombination }\end{array}$ & & \\
\hline T473.7 & & & Yes & JIJ2, EVI20 & NFI & MLPA & $\begin{array}{l}\text { Mitotic } \\
\text { recombination }\end{array}$ & & \\
\hline T473.8 & & & Yes & $\begin{array}{l}\mathrm{HHH} 202, \mathrm{JIJ} 2, \mathrm{EVI} 20, \\
\text { IVS38, 3'NFI, EW207, } \\
\text { DI7S949, DI7SI822 }\end{array}$ & $\begin{array}{l}\mathrm{NFI} \text { and } 3^{\prime} \\
\text { flanking } \\
\text { region }\end{array}$ & MLPA & $\begin{array}{l}\text { Mitotic } \\
\text { recombination }\end{array}$ & & \\
\hline T473.10 & & & Yes & JIJ2, EVI20, IVS38 & NFI & MLPA & $\begin{array}{l}\text { Mitotic } \\
\text { recombination }\end{array}$ & & \\
\hline
\end{tabular}


Table SI. Continued

\begin{tabular}{|c|c|c|c|c|c|c|c|c|c|}
\hline $\begin{array}{l}\text { Patient } \\
\text { ID }\end{array}$ & Germline mutation & $\begin{array}{l}\text { Type of } \\
\text { germline } \\
\text { mutation }\end{array}$ & LOH & LOH markers & $\begin{array}{l}\text { Predicted } \\
\text { extent of } \\
\text { LOH }\end{array}$ & $\begin{array}{l}\text { Evidence } \\
\text { for genomic } \\
\text { deletion? } \\
\text { MLPA/CGH } \\
\text { arrayCGH/ } \\
\text { FISH }\end{array}$ & $\begin{array}{l}\text { Probable } \\
\text { mechanism }\end{array}$ & $\begin{array}{l}\text { No. } \\
\text { samples } \\
\text { with } \\
\text { LOH }\end{array}$ & Reference \\
\hline T473.14 & & & Yes & $\begin{array}{l}\text { JIJ2, EVI20, IVS38, } \\
\text { 3'NFI, EW207, } \\
\text { DI7S949, DI7SI822 }\end{array}$ & $\begin{array}{l}\text { NFI and } 3^{\prime} \\
\text { flanking } \\
\text { region }\end{array}$ & MLPA & $\begin{array}{l}\text { Mitotic } \\
\text { recombination }\end{array}$ & & \\
\hline T473.I5 & & & Yes & $\begin{array}{l}\text { JIJ2, EVI20, IVS38, } \\
3^{\prime} \mathrm{NFI}\end{array}$ & NFI & MLPA & $\begin{array}{l}\text { Mitotic } \\
\text { recombination }\end{array}$ & & \\
\hline T473.16 & & & Yes & $\begin{array}{l}\text { JIJ2, EVI20, IVS38, } \\
\text { 3'NFI, EW207, } \\
\text { DI7S949, } \\
\text { DI7SI822 }\end{array}$ & $\begin{array}{l}\text { NFI and } 3^{\prime} \\
\text { flanking } \\
\text { region }\end{array}$ & MLPA & $\begin{array}{l}\text { Mitotic } \\
\text { recombination }\end{array}$ & & \\
\hline T473.2I & & & Yes & JIJ2, EVI20 & NFI & MLPA & $\begin{array}{l}\text { Mitotic } \\
\text { recombination }\end{array}$ & & \\
\hline T473.35 & & & Yes & EVI20, IVS38 & $\begin{array}{l}\text { Intragenic } \\
\mathrm{NFI}\end{array}$ & MLPA & $\begin{array}{l}\text { Mitotic } \\
\text { recombination }\end{array}$ & & \\
\hline T473.30 & & & Yes & $\begin{array}{l}\text { JIJ2, EVI20, IVS38, } \\
\text { 3'NFI, EW207, } \\
\text { DI7S949 }\end{array}$ & $\begin{array}{l}\mathrm{NFI} \text { and } 3^{\prime} \\
\text { flanking } \\
\text { region }\end{array}$ & MLPA & $\begin{array}{l}\text { Mitotic } \\
\text { recombination }\end{array}$ & & \\
\hline T473.32 & & & Yes & JIJ2, EVI20 & $\begin{array}{l}\text { Intragenic } \\
\mathrm{NFI}\end{array}$ & MLPA & $\begin{array}{l}\text { Mitotic } \\
\text { recombination }\end{array}$ & & \\
\hline T473.34 & & & Yes & EVI20, IVS38 & $\begin{array}{l}\text { Intragenic } \\
\mathrm{NFI}\end{array}$ & MLPA & $\begin{array}{l}\text { Mitotic } \\
\text { recombination }\end{array}$ & & \\
\hline T225. I & ExIOb deletion [MLPA] & $\begin{array}{l}\text { Single exon } \\
\text { deletion }\end{array}$ & & $\mathrm{LOH}$ & & NIA & & & $\begin{array}{l}\text { Unpublished } \\
\text { data, Cardiff }\end{array}$ \\
\hline T225.3 & & & & $\mathrm{LOH}$ & & & & & \\
\hline T68.2 & Deletion exons $10 \mathrm{~b}-19 \mathrm{~b}$ & $\begin{array}{l}\text { Partial } \\
\text { gene } \\
\text { deletion }\end{array}$ & Yes & UTI72 - I38 206,207 & $\begin{array}{l}\text { Exon } 5-3^{\prime} \\
\text { region }\end{array}$ & NIA & & & $\begin{array}{l}\text { Unpublished } \\
\text { data, Cardiff }\end{array}$ \\
\hline T68.3 & & & Yes & UTI72 - I38 206,207 & $\begin{array}{l}\text { Exon 5-3' } \\
\text { region }\end{array}$ & & & & \\
\hline
\end{tabular}


Table SI. Continued

\begin{tabular}{|c|c|c|c|c|c|c|c|c|c|}
\hline $\begin{array}{l}\text { Patient } \\
\text { ID }\end{array}$ & Germline mutation & $\begin{array}{l}\text { Type of } \\
\text { germline } \\
\text { mutation }\end{array}$ & LOH & LOH markers & $\begin{array}{l}\text { Predicted } \\
\text { extent of } \\
\text { LOH }\end{array}$ & $\begin{array}{l}\text { Evidence } \\
\text { for genomic } \\
\text { deletion? } \\
\text { MLPA/CGH } \\
\text { arrayCGH/ } \\
\text { FISH }\end{array}$ & $\begin{array}{l}\text { Probable } \\
\text { mechanism }\end{array}$ & $\begin{array}{l}\text { No. } \\
\text { samples } \\
\text { with } \\
\text { LOH }\end{array}$ & Reference \\
\hline CLJIN & $\begin{array}{l}\text { ExI3: c.204IC > T; } \\
\text { p.Arg68IX }\end{array}$ & Nonsense & Yes & NFI, DI7SI800 & $\begin{array}{l}\text { NFI and } 3^{\prime} \\
\text { flanking } \\
\text { region }\end{array}$ & NIA & & $32 / 126$ & $5,6,7$ \\
\hline CLJ $2 \mathrm{~N}$ & & & Yes & $\begin{array}{l}\text { DI7S33, DI7SI294, } \\
\text { NFI, DI7SI800, } \\
\text { DI7S798, DI7S250, } \\
\text { DI7S787, DI7S802 }\end{array}$ & $\begin{array}{l}\text { Majority } \\
\text { of } 17 q\end{array}$ & & & & \\
\hline TI70.3 & $\begin{array}{l}\text { ExI3: c.204IC > T; } \\
\text { p.Arg68IX }\end{array}$ & Nonsense & & $\mathrm{LOH}$ & & NIA & & & $\begin{array}{l}\text { Unpublished } \\
\text { data, Cardiff }\end{array}$ \\
\hline TI70.2 & & & & $\mathrm{LOH}$ & & & & & \\
\hline ABAIN & ExI3: c. $225 \mathrm{I}+2 \mathrm{~T}>\mathrm{C}$ & Splice site & Yes & $\begin{array}{l}\text { DSI7SI824, DI7S84I, } \\
\text { DI7SI294, DI7SI863, } \\
\text { NFI, DI7SI800, } \\
\text { DI7SI880, DI7S798, } \\
\text { DI7S250, DI7S787, } \\
\text { DI7S802, DI7S784, } \\
\text { DI7S928 }\end{array}$ & $\begin{array}{l}\text { Majority } \\
\text { of } 17 q\end{array}$ & NIA & & $32 / 126$ & $5,6,7$ \\
\hline ABA2N & & & Yes & $\begin{array}{l}\text { DSI7SI824, DI7S84I, } \\
\text { DI7SI294, DI7SI863, } \\
\text { NFI, DI7SI800, } \\
\text { DI7SI880, DI7S798, } \\
\text { DI7S250, DI7S787, } \\
\text { DI7S802, DI7S784, } \\
\text { DI7S928 }\end{array}$ & $\begin{array}{l}\text { Majority } \\
\text { of } 17 q\end{array}$ & & & & $5,6,7$ \\
\hline T436 & $\begin{array}{l}\text { ExI7: c. } 2875 C>T \text {; } \\
\text { p.Glu959X }\end{array}$ & Nonsense & Yes & IVS27, EVI20, IVS38 & $\begin{array}{l}\text { Intragenic } \\
\text { NFI }\end{array}$ & MLPA & $\begin{array}{l}\text { Mitotic } \\
\text { recombination }\end{array}$ & $22 / 89$ & 4 \\
\hline T439 & & & Yes & IVS27, EVI20, IVS38 & $\begin{array}{l}\text { Intragenic } \\
\mathrm{NFI}\end{array}$ & MLPA & $\begin{array}{l}\text { Mitotic } \\
\text { recombination }\end{array}$ & & \\
\hline T440 & & & Yes & IVS27, EVI20, IVS38 & $\begin{array}{l}\text { Intragenic } \\
\mathrm{NFI}\end{array}$ & MLPA & $\begin{array}{l}\text { Mitotic } \\
\text { recombination }\end{array}$ & & \\
\hline
\end{tabular}




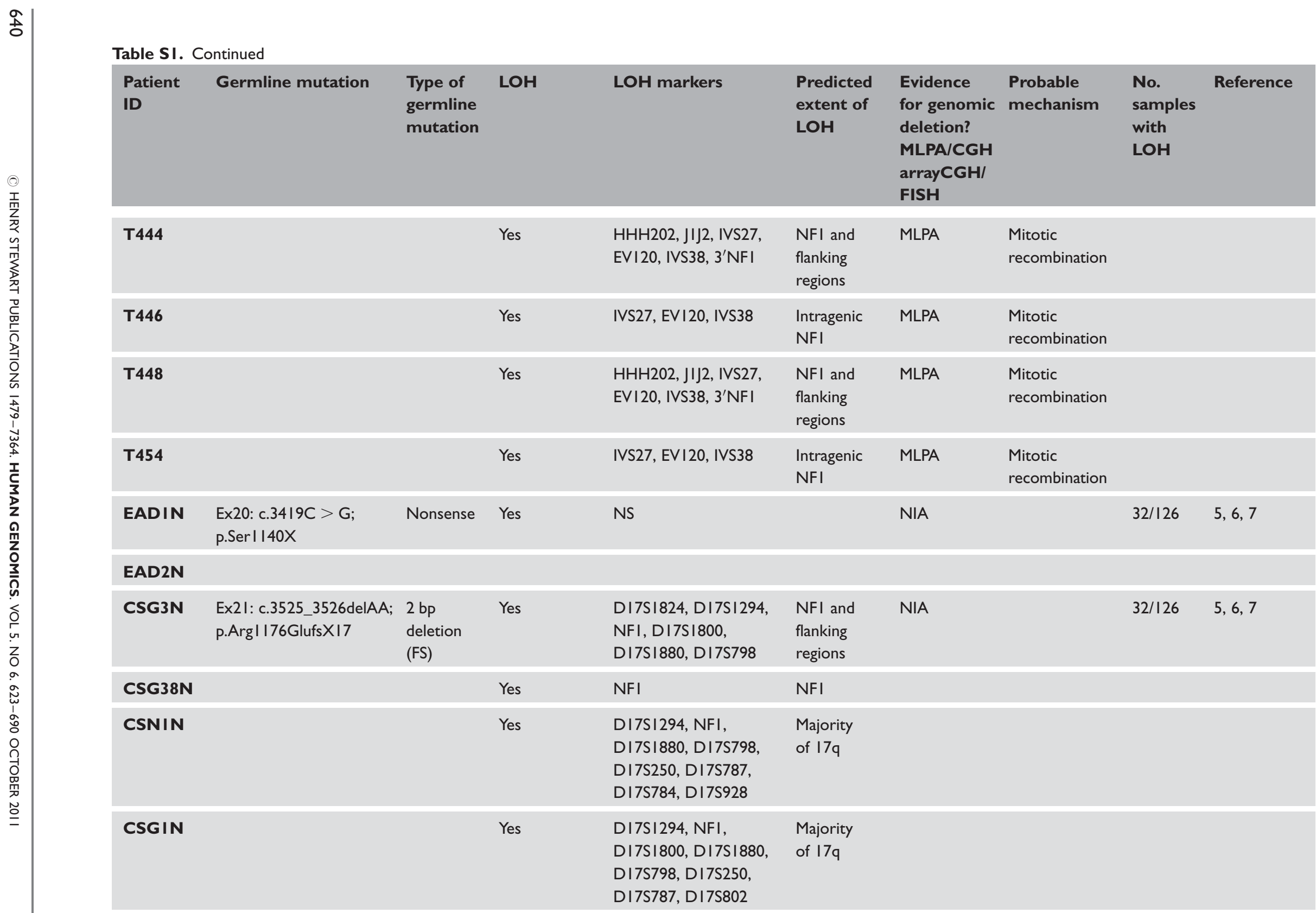


Table SI. Continued

\begin{tabular}{|c|c|c|c|c|c|c|c|c|c|}
\hline $\begin{array}{l}\text { Patient } \\
\text { ID }\end{array}$ & Germline mutation & $\begin{array}{l}\text { Type of } \\
\text { germline } \\
\text { mutation }\end{array}$ & LOH & LOH markers & $\begin{array}{l}\text { Predicted } \\
\text { extent of } \\
\text { LOH }\end{array}$ & $\begin{array}{l}\text { Evidence } \\
\text { for genomic } \\
\text { deletion? } \\
\text { MLPA/CGH } \\
\text { arrayCGH/ } \\
\text { FISH }\end{array}$ & $\begin{array}{l}\text { Probable } \\
\text { mechanism }\end{array}$ & $\begin{array}{l}\text { No. } \\
\text { samples } \\
\text { with } \\
\text { LOH }\end{array}$ & Reference \\
\hline CSG2N & & & Yes & $\begin{array}{l}\text { DSI7SI824, DI7SI294, } \\
\text { NFI, DI7SI800, } \\
\text { DI7SI880, DI7S798, } \\
\text { DI7S250, DI7S787, } \\
\text { DI7S802 }\end{array}$ & $\begin{array}{l}\text { Majority } \\
\text { of } 17 q\end{array}$ & & & & \\
\hline CSG4N & & & Yes & $\begin{array}{l}\text { DSI7SI824, DI7SI294, } \\
\text { NFI, DI7SI800, } \\
\text { DI7SI880, DI7S798, } \\
\text { DI7S250, DI7S787, } \\
\text { DI7S802 }\end{array}$ & $\begin{array}{l}\text { Majority } \\
\text { of } 17 q\end{array}$ & & & & \\
\hline CSG5N & & & Yes & $\begin{array}{l}\text { DSI7SI824, DI7SI294, } \\
\text { NFI, DI7SI800, } \\
\text { DI7SI880, DI7S798, } \\
\text { DI7S250, DI7S787, } \\
\text { DI7S802 }\end{array}$ & $\begin{array}{l}\text { Majority } \\
\text { of } 17 q\end{array}$ & & & & \\
\hline CSG2IN & & & Yes & $\begin{array}{l}\text { DSI7SI824, DI7SI294, } \\
\text { NFI, DI7SI800, } \\
\text { DI7SI880, DI7S798, } \\
\text { DI7S250, DI7S787, } \\
\text { DI7S802 }\end{array}$ & $\begin{array}{l}\text { Majority } \\
\text { of } 17 q\end{array}$ & & & & \\
\hline CSG25N & & & Yes & $\begin{array}{l}\text { DI7SI294, NFI, } \\
\text { DI7SI800, DI7SI880, } \\
\text { DI7S798, DI7S250, } \\
\text { DI7S787, DI7S802 }\end{array}$ & $\begin{array}{l}\text { Majority } \\
\text { of } 17 q\end{array}$ & & & & \\
\hline CSG42N & & & Yes & $\begin{array}{l}\text { DI7SI294, NFI, } \\
\text { DI7SI800, DI7SI880, } \\
\text { DI7S798, DI7S250, } \\
\text { DI7S787, DI7S802 }\end{array}$ & $\begin{array}{l}\text { Majority } \\
\text { of } 17 q\end{array}$ & & & & \\
\hline
\end{tabular}


Table SI. Continued

\begin{tabular}{|c|c|c|c|c|c|c|c|c|c|}
\hline $\begin{array}{l}\text { Patient } \\
\text { ID }\end{array}$ & Germline mutation & $\begin{array}{l}\text { Type of } \\
\text { germline } \\
\text { mutation }\end{array}$ & LOH & LOH markers & $\begin{array}{l}\text { Predicted } \\
\text { extent of } \\
\text { LOH }\end{array}$ & $\begin{array}{l}\text { Evidence } \\
\text { for genomic } \\
\text { deletion? } \\
\text { MLPA/CGH } \\
\text { arrayCGH/ } \\
\text { FISH }\end{array}$ & $\begin{array}{l}\text { Probable } \\
\text { mechanism }\end{array}$ & $\begin{array}{l}\text { No. } \\
\text { samples } \\
\text { with } \\
\text { LOH }\end{array}$ & Reference \\
\hline CSG5IN & & & Yes & $\begin{array}{l}\text { DSI7S1824, DI7SI294, } \\
\text { NFI, DI7SI800, } \\
\text { DI7SI880, DI7S798, } \\
\text { DI7S250, DI7S787, } \\
\text { DI7S802 }\end{array}$ & $\begin{array}{l}\text { Majority } \\
\text { of } 17 q\end{array}$ & FISH & $\begin{array}{l}\text { Mitotic } \\
\text { recombination }\end{array}$ & & \\
\hline CSG52N & & & Yes & $\begin{array}{l}\text { DI7S33, DSI7SI824, } \\
\text { DI7SI294, NFI, } \\
\text { DI7SI800, DI7SI880, } \\
\text { DI7S798, DI7S250, } \\
\text { DI7S787, DI7S802 }\end{array}$ & $\begin{array}{l}\text { Majority } \\
\text { of } 17 q\end{array}$ & NIA & & & \\
\hline CSG62N & & & Yes & NS & & & & & \\
\hline TI77 & $\begin{array}{l}\text { Ex23.I: c.3916 C > T; } \\
\text { p.ArgI306X }\end{array}$ & Nonsense & & $\mathrm{LOH}$ & & NIA & & & $\begin{array}{l}\text { Unpublished } \\
\text { data, Cardiff }\end{array}$ \\
\hline T213 & & & & LOH & & & & & \\
\hline $\begin{array}{l}\text { NF44 } \\
\text { UHG_I }\end{array}$ & Ex27a: c.45I5-2A > T & Splice site & Yes & $\begin{array}{l}\text { NFI germline mutation, } \\
\text { rs } 9891455\end{array}$ & NFI & Array CGH & Deletion & $6 / 28$ & 2 \\
\hline $\begin{array}{l}\text { NF44 } \\
\text { UHG_4I }\end{array}$ & & & Yes & rs 1018190 & NFI & Array CGH & Deletion & & \\
\hline T106.I & $\begin{array}{l}\text { Ex37: c.679I dupA; } \\
\text { p.Tyr2264X }\end{array}$ & $\begin{array}{l}\text { I bp } \\
\text { insertion } \\
\text { (FS) }\end{array}$ & Yes & IVS38, $3^{\prime} \mathrm{NFI-I}$ & & NIA & & & $\begin{array}{l}\text { Unpublished } \\
\text { data, Cardiff }\end{array}$ \\
\hline T106.5 & & & Yes & $14 \mid-C 3$ & $3^{\prime}$ UTR & & & & \\
\hline T106.6 & & & Yes & $\begin{array}{l}\text { JIJ2, EVI20, I38, 14I, } \\
\text { C3C7, 206, } 207\end{array}$ & & & & & \\
\hline T210.2 & $\begin{array}{l}\text { Ex42: c.7458delC; } \\
\text { p.Tyr2487llefs X5 }\end{array}$ & $\begin{array}{l}\text { I bp } \\
\text { deletion } \\
\text { (FS) }\end{array}$ & & & & $30 \%$ WG & & & $\begin{array}{l}\text { Unpublished } \\
\text { data, Cardiff }\end{array}$ \\
\hline
\end{tabular}


Table SI. Continued

\begin{tabular}{|c|c|c|c|c|c|c|c|c|c|}
\hline $\begin{array}{l}\text { Patient } \\
\text { ID }\end{array}$ & Germline mutation & $\begin{array}{l}\text { Type of } \\
\text { germline } \\
\text { mutation }\end{array}$ & LOH & LOH markers & $\begin{array}{l}\text { Predicted } \\
\text { extent of } \\
\text { LOH }\end{array}$ & $\begin{array}{l}\text { Evidence } \\
\text { for genomic } \\
\text { deletion? } \\
\text { MLPA/CGH } \\
\text { arrayCGH/ } \\
\text { FISH }\end{array}$ & $\begin{array}{l}\text { Probable } \\
\text { mechanism }\end{array}$ & $\begin{array}{l}\text { No. } \\
\text { samples } \\
\text { with } \\
\text { LOH }\end{array}$ & Reference \\
\hline T210.4 & & & & & & $30 \%$ WG & & & \\
\hline T210.8 & & & & & & $30 \%$ WG & & & \\
\hline T210.4 & & & & & & E8: $30 \%$ WG & & & \\
\hline T210.5 & & & & & & $\begin{array}{l}\text { E8: exon } \\
\text { duplication }\end{array}$ & & & \\
\hline HTI 335 & $\begin{array}{l}\text { Ex4c: c. } 7237 C>T ; \\
\text { p.Gln } 2413 X\end{array}$ & Nonsense & & & & EI6: del & & & $\begin{array}{l}\text { Unpublished } \\
\text { data, Cardiff }\end{array}$ \\
\hline TI 28.30 & $\begin{array}{l}\text { Ex6: c. } 784 C>T ; \\
\text { p.Arg } 262 \text { Cys }\end{array}$ & Missense & Yes & & $\begin{array}{l}3^{\prime} \text { UTR to } \\
3^{\prime} \text { region }\end{array}$ & NIA & & & $\begin{array}{l}\text { Unpublished } \\
\text { data, Cardiff }\end{array}$ \\
\hline TI92.4 & $\begin{array}{l}\text { Deletion of exons } \\
6-27 a \text { [MLPA] }\end{array}$ & $\begin{array}{l}\text { Multi-exon } \\
\text { deletion }\end{array}$ & & $\begin{array}{l}\text { LOH: JIJ2, EVI20, } \\
\mathrm{HHH} 202 \text {, }\end{array}$ & & & & & $\begin{array}{l}\text { Unpublished } \\
\text { data, Cardiff }\end{array}$ \\
\hline p062 & $\begin{array}{l}\text { Ex7: c.910C > T; } \\
\text { p.Arg304X }\end{array}$ & Nonsense & & LOH (6 samples) & & $\begin{array}{l}\text { Deletion } \\
\text { (2 samples) }\end{array}$ & & & $\begin{array}{l}\text { Unpublished } \\
\text { data, Cardiff }\end{array}$ \\
\hline p082 & $\begin{array}{l}\text { Ex7: c.910C > T; } \\
\text { p.Arg304X }\end{array}$ & Nonsense & & LOH (5 samples) & & $\begin{array}{l}\text { Deletion } \\
\text { (5 samples) }\end{array}$ & & & \\
\hline ACFIN & $\begin{array}{l}\text { Ex7: c.910C > T; } \\
\text { p.Arg304X }\end{array}$ & Nonsense & Yes & $\begin{array}{l}\text { DI7S84I, DI7SI294, } \\
\text { DI7SI863, NFI, } \\
\text { DI7SI880, DI7S798, } \\
\text { DI7S250, DI7S802, } \\
\text { DI7S784 }\end{array}$ & $\begin{array}{l}\text { Majority } \\
\text { of } 17 q\end{array}$ & FISH & $\begin{array}{l}\text { Mitotic } \\
\text { recombination }\end{array}$ & $32 / 126$ & $5,6,7$ \\
\hline MIGSIN & $\begin{array}{l}\text { Ex7: c.910C > T; } \\
\text { p.Arg304X }\end{array}$ & Nonsense & Yes & $\begin{array}{l}\text { DI7I863, NFI, } \\
\text { DI7SI800, DI7SI880 }\end{array}$ & $\begin{array}{l}\mathrm{NFI} \text { and } \\
\text { flanking } \\
\text { regions }\end{array}$ & NIA & & $32 / 126$ & \\
\hline CAGIN & $\begin{array}{l}\text { Ex7: c.979delCinsTT; } \\
\text { p.Leu327Phefs X3 }\end{array}$ & Indel (FS) & Yes & $\mathrm{NFI}$ & $\begin{array}{l}\text { Intragenic } \\
\mathrm{NFI}\end{array}$ & NIA & & $32 / 126$ & \\
\hline CAG3N & $\begin{array}{l}\text { Ex7: c.979delCinsTT; } \\
\text { p.Leu327PhefsX3 }\end{array}$ & Indel (FS) & Yes & NS & & NIA & & $32 / 126$ & \\
\hline
\end{tabular}


Table SI. Continued

\begin{tabular}{|c|c|c|c|c|c|c|c|c|c|}
\hline $\begin{array}{l}\text { Patient } \\
\text { ID }\end{array}$ & Germline mutation & $\begin{array}{l}\text { Type of } \\
\text { germline } \\
\text { mutation }\end{array}$ & LOH & LOH markers & $\begin{array}{l}\text { Predicted } \\
\text { extent of } \\
\text { LOH }\end{array}$ & $\begin{array}{l}\text { Evidence } \\
\text { for genomic } \\
\text { deletion? } \\
\text { MLPA/CGH } \\
\text { arrayCGH/ } \\
\text { FISH }\end{array}$ & $\begin{array}{l}\text { Probable } \\
\text { mechanism }\end{array}$ & $\begin{array}{l}\text { No. } \\
\text { samples } \\
\text { with } \\
\text { LOH }\end{array}$ & Reference \\
\hline T199 & $\begin{array}{l}\text { Ex7: c.983_984delGT; } \\
\text { p.Cys324X }\end{array}$ & $\begin{array}{l}2 \text { bp } \\
\text { deletion } \\
\text { (FS) }\end{array}$ & Yes & IVSI2, JIJ2 & & NIA & & & $\begin{array}{l}\text { Unpublished } \\
\text { data, Cardiff }\end{array}$ \\
\hline NF56-2 & $\begin{array}{l}\text { Ex9: c. } 1246 \mathrm{C}>\mathrm{T} \text {; } \\
\text { p.Arg416X }\end{array}$ & Missense & Yes & $\begin{array}{l}\text { Pin I, Rsal, Alul, Pin 28, } \\
530, \text { NFI 3'UTR, Mfd } \\
\text { I5 }\end{array}$ & $\begin{array}{l}\text { NFI and } \\
\text { flanking } \\
\text { regions }\end{array}$ & NIA & & $1 / 6$ & 8 \\
\hline TI97A & $\begin{array}{l}\text { ExI0a: c. } 1318 \mathrm{C}>\mathrm{T} \\
\text { p.Arg440X }\end{array}$ & Nonsense & & $\mathrm{LOH}$ ? & & & & & $\begin{array}{l}\text { Unpublished } \\
\text { data, Cardiff }\end{array}$ \\
\hline CLTIN & $\begin{array}{l}\text { ExI2a: } \\
\text { c.I754_I757delTAAC; } \\
\text { p.Thr586SerfsXI9 }\end{array}$ & $\begin{array}{l}4 \text { bp } \\
\text { deletion } \\
\text { (FS) }\end{array}$ & Yes & $\begin{array}{l}\text { DI7S84I, DI7SI863, } \\
\text { NFI, DI7SI800, } \\
\text { DI7SI880, DI7S787, } \\
\text { DI7S802 }\end{array}$ & $\begin{array}{l}\text { Majority } \\
\text { of } 17 q\end{array}$ & & & $32 / 126$ & \\
\hline p022 & $\begin{array}{l}\text { ExI2a: } \\
\text { c.I756_I759delACTA; } \\
\text { p.Thr586ValfsXI8 }\end{array}$ & $\begin{array}{l}4 \text { bp } \\
\text { deletion } \\
\text { (FS) }\end{array}$ & & LOH (9 samples) & & $\begin{array}{l}\text { Deletion } \\
\text { (2 samples) }\end{array}$ & & & $\begin{array}{l}\text { Unpublished } \\
\text { data, Cardiff }\end{array}$ \\
\hline p020 & $\begin{array}{l}\text { ExI3: c.204IC > T; } \\
\text { p.Arg68IX }\end{array}$ & Nonsense & & LOH (2 samples) & & $\begin{array}{l}\text { Deletion } \\
\text { (0 samples) }\end{array}$ & & & \\
\hline TI4I.5 & $\begin{array}{l}\text { ExI3: c.2233delA; } \\
\text { p.Ser745AlafsX2 }\end{array}$ & $\begin{array}{l}\text { I bp } \\
\text { deletion } \\
\text { (FS) }\end{array}$ & Yes & $\begin{array}{l}\text { 202, I 2b, IVS27, IVS38, } \\
3^{\prime} \mathrm{NFI}\end{array}$ & & & & & $\begin{array}{l}\text { Unpublished } \\
\text { data, Cardiff }\end{array}$ \\
\hline plo3 & $\begin{array}{l}\text { ExI5: c. } 2338 \mathrm{~A}>\mathrm{C} \text {; } \\
\text { p.Thr780Pro }\end{array}$ & Missense & & LOH (I0 samples) & & $\begin{array}{l}\text { Deletion } \\
\text { (3 samples) }\end{array}$ & & & $\begin{array}{l}\text { Unpublished } \\
\text { data, Cardiff }\end{array}$ \\
\hline T22 & ExI7: c. $285 I-2 A>G$ & Splice site & Yes & & $\begin{array}{l}3^{\prime} \cup T R \text { to } 3^{\prime} \\
\text { flanking } \\
\text { regions }\end{array}$ & & & & $\begin{array}{l}\text { Unpublished } \\
\text { data, Cardiff }\end{array}$ \\
\hline $\begin{array}{l}\text { NF253- } \\
\text { UHG E }\end{array}$ & ExI7: c. $285 I-2 A>G$ & Splice site & Yes & Not specific & & NIA & & $3 / 38$ & 3 \\
\hline L005 A & Ex|8: c. $3113+I G>A$ & Splice site & & & & & & & \\
\hline
\end{tabular}




\begin{tabular}{|c|c|c|c|c|c|c|c|c|c|}
\hline $\begin{array}{l}\text { Patient } \\
\text { ID }\end{array}$ & Germline mutation & $\begin{array}{l}\text { Type of } \\
\text { germline } \\
\text { mutation }\end{array}$ & LOH & LOH markers & $\begin{array}{l}\text { Predicted } \\
\text { extent of } \\
\text { LOH }\end{array}$ & $\begin{array}{l}\text { Evidence } \\
\text { for genomic } \\
\text { deletion? } \\
\text { MLPA/CGH } \\
\text { arrayCGH/ } \\
\text { FISH }\end{array}$ & $\begin{array}{l}\text { Probable } \\
\text { mechanism }\end{array}$ & $\begin{array}{l}\text { No. } \\
\text { samples } \\
\text { with } \\
\text { LOH }\end{array}$ & Reference \\
\hline 319TI & $\begin{array}{l}\text { ExI9b: c. } 3208 C>T \text {; } \\
\text { p.GInI070X }\end{array}$ & Nonsense & Yes & NF-exon5 & $\begin{array}{l}\text { Intragenic } \\
\mathrm{NFI}\end{array}$ & & & $2 / 15$ & 9 \\
\hline p023 & $\begin{array}{l}\text { Ex2I: c.3525_3526delAA; } \\
\text { p.ArgII } 76 \text { SerfsX18 }\end{array}$ & $\begin{array}{l}2 \text { bp } \\
\text { deletion } \\
\text { (FS) }\end{array}$ & & LOH (I4 samples) & & $\begin{array}{l}\text { Deletion } \\
\text { (5 samples) }\end{array}$ & & & $\begin{array}{l}\text { Unpublished } \\
\text { data, Cardiff }\end{array}$ \\
\hline pOII & $\begin{array}{l}\text { Ex22: c. } 3826 C>T ; \\
\text { p.Argl } 276 X\end{array}$ & Nonsense & & LOH (5 samples) & & $\begin{array}{l}\text { Deletion } \\
\text { (I sample) }\end{array}$ & & & \\
\hline MASG2N & Ex22: c.3870 + IG > T & Splice site & Yes & $\begin{array}{l}\text { NFI, DI7SI880, } \\
\text { DI7S798, DI7S250, } \\
\text { DI7S787 }\end{array}$ & $\begin{array}{l}\text { Majority } \\
\text { of } 17 q\end{array}$ & FISH & $\begin{array}{l}\text { Mitotic } \\
\text { recombination }\end{array}$ & $32 / 126$ & $5,6,7$ \\
\hline TI7I & $\begin{array}{l}\text { Ex23.2: c. } 4084 \mathrm{C}>\mathrm{T} \text {; } \\
\text { p.Argl } 362 \mathrm{X}\end{array}$ & Nonsense & & $\mathrm{LOH}$ & & & & & $\begin{array}{l}\text { Unpublished } \\
\text { data, Cardiff }\end{array}$ \\
\hline plo4 & $\begin{array}{l}\text { Ex25: c. } 4309 \mathrm{G}>\mathrm{T} \text {; } \\
\text { p.Glul } 436 \mathrm{X}\end{array}$ & Nonsense & & LOH (3 samples) & & $\begin{array}{l}\text { Deletion } \\
\text { (0 samples) }\end{array}$ & & & $\begin{array}{l}\text { Unpublished } \\
\text { data, Cardiff }\end{array}$ \\
\hline p078 & $\begin{array}{l}\text { Ex27a: c. } 4537 C>T \text {; } \\
\text { p.Argl5।3X }\end{array}$ & Nonsense & & LOH (6 samples) & & $\begin{array}{l}\text { Deletion } \\
\text { (0 samples) }\end{array}$ & & & \\
\hline p084 & $\begin{array}{l}\text { Ex27a: c. } 4572 C>\text { G; } \\
\text { p.TyrI524X }\end{array}$ & Nonsense & & LOH (2 samples) & & $\begin{array}{l}\text { Deletion } \\
\text { (0 samples) }\end{array}$ & & & \\
\hline pl02 & $\begin{array}{l}\text { Ex29: c. } 5242 C>T ; \\
\text { p.Argl } 748 X\end{array}$ & Nonsense & & LOH (5 samples) & & $\begin{array}{l}\text { Deletion } \\
\text { (2 samples) }\end{array}$ & & & \\
\hline p055 & $\begin{array}{l}\text { Ex30: c. } 5710 \mathrm{G}>\mathrm{T} \text {; } \\
\text { p.Glul } 904 \mathrm{X}\end{array}$ & Nonsense & & LOH (I sample) & & $\begin{array}{l}\text { Deletion } \\
\text { (0 samples) }\end{array}$ & & & \\
\hline EMNIN & $\begin{array}{l}\text { Ex30: } \\
c .5749+332 A>G\end{array}$ & Splice site & Yes & NFI, DI7SI800 & $\begin{array}{l}\mathrm{NFI} \text { and } 3^{\prime} \\
\text { flanking } \\
\text { region }\end{array}$ & & & $32 / 126$ & $5,6,7$ \\
\hline
\end{tabular}


Table SI. Continued

\begin{tabular}{|c|c|c|c|c|c|c|c|c|c|}
\hline $\begin{array}{l}\text { Patient } \\
\text { ID }\end{array}$ & Germline mutation & $\begin{array}{l}\text { Type of } \\
\text { germline } \\
\text { mutation }\end{array}$ & LOH & LOH markers & $\begin{array}{l}\text { Predicted } \\
\text { extent of } \\
\text { LOH }\end{array}$ & $\begin{array}{l}\text { Evidence } \\
\text { for genomic } \\
\text { deletion? } \\
\text { MLPA/CGH } \\
\text { arrayCGH/ } \\
\text { FISH }\end{array}$ & $\begin{array}{l}\text { Probable } \\
\text { mechanism }\end{array}$ & $\begin{array}{l}\text { No. } \\
\text { samples } \\
\text { with } \\
\text { LOH }\end{array}$ & Reference \\
\hline p027 & $\begin{array}{l}\text { Ex33: c.6226delG; } \\
\text { p.Ala2076GInfsXI3 }\end{array}$ & $\begin{array}{l}\text { I bp } \\
\text { deletion } \\
\text { (FS) }\end{array}$ & & LOH (I sample) & & $\begin{array}{l}\text { Deletion } \\
\text { (0 samples) }\end{array}$ & & & $\begin{array}{l}\text { Unpublished } \\
\text { data, Cardiff }\end{array}$ \\
\hline p052 & $\begin{array}{l}\text { Ex37: c.679I_6792dupA; } \\
\text { p.Tyr2264X }\end{array}$ & $\begin{array}{l}\text { I bp } \\
\text { duplication } \\
\text { (FS) }\end{array}$ & & LOH (I sample) & & $\begin{array}{l}\text { Deletion } \\
\text { (I sample) }\end{array}$ & & & \\
\hline T23.6 & $\begin{array}{l}\text { Ex4I: } \\
\text { c.7268_7269delCA; } \\
\text { p.Thr2423SerfsX2 }\end{array}$ & $\begin{array}{l}2 \text { bp } \\
\text { deletion } \\
\text { (FS) }\end{array}$ & Yes & EVI20, I38, 14I, C3 & & NIA & & & $\begin{array}{l}\text { Unpublished } \\
\text { data, Cardiff }\end{array}$ \\
\hline T100 & $\begin{array}{l}\text { Ex4l: c. } 7267 \text { dupA; } \\
\text { p.Thr2426X }\end{array}$ & $\begin{array}{l}\text { I bp } \\
\text { duplication } \\
\text { (FS) }\end{array}$ & Yes & & $\begin{array}{l}\text { I38 to } \\
3^{\prime} \cup T R\end{array}$ & & & & $\begin{array}{l}\text { Unpublished } \\
\text { data, Cardiff }\end{array}$ \\
\hline TI64.I & $\begin{array}{l}\text { Ex4l: c. } 7285 \text { C }>\text { T; } \\
\text { p.Arg2429X }\end{array}$ & Nonsense & & $\mathrm{LOH}$ & & & & & $\begin{array}{l}\text { Unpublished } \\
\text { data, Cardiff }\end{array}$ \\
\hline MAR2N & $\mathrm{NI}$ & $\mathrm{NI}$ & Yes & NFI, DI7SI800 & $\begin{array}{l}\mathrm{NFI} \text { and } 3^{\prime} \\
\text { flanking } \\
\text { region }\end{array}$ & NIA & & $32 / 126$ & $5,6,7$ \\
\hline MOPT2N & $\mathrm{NI}$ & $\mathrm{NI}$ & Yes & $\begin{array}{l}\text { DI7SI824, DI7SI294, } \\
\text { DI7I863, NFI, } \\
\text { DI7SI800, DI7SI880 }\end{array}$ & $\begin{array}{l}\text { NFI and } \\
\text { flanking } \\
\text { regions }\end{array}$ & & & & \\
\hline NGLIN & $\mathrm{NI}$ & $\mathrm{NI}$ & Yes & $\begin{array}{l}\text { DI7S84I, DI7SI294, } \\
\text { NFI, DI7SI800, } \\
\text { DI7SI880, DI7S798, } \\
\text { DI7S250, DI7S802, } \\
\text { DI7S784, DI7S928 }\end{array}$ & $\begin{array}{l}\text { Majority } \\
\text { of } 17 q\end{array}$ & & & & \\
\hline JRR2N & $\mathrm{NI}$ & $\mathrm{NI}$ & Yes & $\begin{array}{l}\text { DI7SI 294, DI7SI863, } \\
\text { NFI, DI7SI800, } \\
\text { DI7SI880, DI7S798, } \\
\text { DI7S250, DI7S787 }\end{array}$ & $\begin{array}{l}\text { Majority } \\
\text { of } 17 q\end{array}$ & & & & \\
\hline
\end{tabular}


Table SI. Continued

\begin{tabular}{|c|c|c|c|c|c|c|c|c|c|}
\hline $\begin{array}{l}\text { Patient } \\
\text { ID }\end{array}$ & Germline mutation & $\begin{array}{l}\text { Type of } \\
\text { germline } \\
\text { mutation }\end{array}$ & LOH & LOH markers & $\begin{array}{l}\text { Predicted } \\
\text { extent of } \\
\text { LOH }\end{array}$ & $\begin{array}{l}\text { Evidence } \\
\text { for genomic } \\
\text { deletion? } \\
\text { MLPA/CGH } \\
\text { arrayCGH/ } \\
\text { FISH }\end{array}$ & $\begin{array}{l}\text { Probable } \\
\text { mechanism }\end{array}$ & $\begin{array}{l}\text { No. } \\
\text { samples } \\
\text { with } \\
\text { LOH }\end{array}$ & Reference \\
\hline SLCIN & $\mathrm{NI}$ & $\mathrm{NI}$ & Yes & $\begin{array}{l}\text { DI7S33, DSI7SI824, } \\
\text { DI7S84I, DI7SI294, } \\
\text { NFI, DI7SI880, } \\
\text { DI7S798, DI7S250, } \\
\text { DI7S784, DI7S928 }\end{array}$ & $\begin{array}{l}\text { Majority } \\
\text { of } 17 q\end{array}$ & & & & \\
\hline HTI377.I & $\mathrm{NI}$ & $\mathrm{NI}$ & Yes & & & & & & $\begin{array}{l}\text { Unpublished } \\
\text { data, Cardiff }\end{array}$ \\
\hline HTI377.2 & $\mathrm{NI}$ & $\mathrm{NI}$ & Yes & & & NIA & & & \\
\hline T109.4 & $\mathrm{NI}$ & $\mathrm{NI}$ & Yes & I38, 14I, 206 & $3^{\prime}$ region & & & & \\
\hline TI67.C & $\mathrm{NI}$ & $\mathrm{NI}$ & Yes & IVS27, IVS38, 3’NFI & & & & & \\
\hline TI92.I & $\mathrm{NI}$ & $\mathrm{NI}$ & Yes & 202, IVSI2, JIJ2, IVS27 & & & & & \\
\hline T197 & $\mathrm{NI}$ & $\mathrm{NI}$ & Yes & JIJ2 & & & & & \\
\hline T227.2 & $\mathrm{NI}$ & $\mathrm{NI}$ & Yes & IVSI2, JIJ2 & & & & & \\
\hline T230.2 & $\mathrm{NI}$ & $\mathrm{NI}$ & Yes & 202, IVSI2, IVS27 & & & & & \\
\hline T232.2 & $\mathrm{NI}$ & $\mathrm{NI}$ & Yes & JIJ2 & & & & & \\
\hline T24I & $\mathrm{NI}$ & $\mathrm{NI}$ & Yes & JIJ2 & & & & & \\
\hline NF253_32 & $\mathrm{NI}$ & $\mathrm{NI}$ & Yes & $\begin{array}{l}\text { rsl0|8190, rs989|455, } \\
\text { rs807406I }\end{array}$ & $\mathrm{NFI}$ & Array CGH & $\begin{array}{l}\text { Mitotic } \\
\text { recombination }\end{array}$ & $6 / 28$ & 2 \\
\hline T224.I & $\mathrm{NI}$ & $\mathrm{NI}$ & & $\mathrm{LOH}$ & & NIA & & & $\begin{array}{l}\text { Unpublished } \\
\text { data, Cardiff }\end{array}$ \\
\hline T162 & $\mathrm{NI}$ & $\mathrm{NI}$ & & $\mathrm{LOH}$ & & & & & \\
\hline TI72 & $\mathrm{NI}$ & $\mathrm{NI}$ & & $\mathrm{LOH}$ & & & & & \\
\hline \multirow[t]{2}{*}{ TI79.I } & $\mathrm{NI}$ & $\mathrm{NI}$ & & $\mathrm{LOH}$ & & & & & \\
\hline & & & & & & & & & Continued \\
\hline
\end{tabular}


Table SI. Continued

\begin{tabular}{|c|c|c|c|c|c|c|c|c|c|}
\hline $\begin{array}{l}\text { Patient } \\
\text { ID }\end{array}$ & Germline mutation & $\begin{array}{l}\text { Type of } \\
\text { germline } \\
\text { mutation }\end{array}$ & LOH & LOH markers & $\begin{array}{l}\text { Predicted } \\
\text { extent of } \\
\text { LOH }\end{array}$ & $\begin{array}{l}\text { Evidence } \\
\text { for genomic } \\
\text { deletion? } \\
\text { MLPA/CGH } \\
\text { arrayCGH/ } \\
\text { FISH }\end{array}$ & $\begin{array}{l}\text { Probable } \\
\text { mechanism }\end{array}$ & $\begin{array}{l}\text { No. } \\
\text { samples } \\
\text { with } \\
\text { LOH }\end{array}$ & Reference \\
\hline
\end{tabular}

\begin{tabular}{|c|c|c|c|}
\hline T204.2 & $\mathrm{NI}$ & $\mathrm{NI}$ & $\mathrm{LOH}$ \\
\hline T224.2 & $\mathrm{NI}$ & $\mathrm{NI}$ & $\mathrm{LOH}$ \\
\hline TI73.I & $\mathrm{NI}$ & $\mathrm{NI}$ & $\mathrm{LOH}$ \\
\hline T179.2 & $\mathrm{NI}$ & $\mathrm{NI}$ & LOH \\
\hline TI28I.2 & $\mathrm{NI}$ & $\mathrm{NI}$ & $\mathrm{LOH}$ \\
\hline TI28I.4 & $\mathrm{NI}$ & $\mathrm{NI}$ & LOH \\
\hline T220 & $\mathrm{NI}$ & $\mathrm{NI}$ & $\mathrm{LOH}$ \\
\hline T22I & $\mathrm{NI}$ & $\mathrm{NI}$ & $\mathrm{LOH}$ \\
\hline T223 & $\mathrm{NI}$ & $\mathrm{NI}$ & $\mathrm{LOH}$ \\
\hline T258.I & $\mathrm{NI}$ & $\mathrm{NI}$ & $\mathrm{LOH}$ \\
\hline T258.2 & $\mathrm{NI}$ & $\mathrm{NI}$ & $\mathrm{LOH}$ \\
\hline
\end{tabular}

\section{SCs from cutaneous neurofibromas}

\begin{tabular}{|c|c|c|c|c|c|c|}
\hline T543.2 & $\begin{array}{l}\text { Ex4a: } \\
\text { c.373delGinsATGTGT; } \\
\text { p.ArgI25HisfsX4 }\end{array}$ & Indel (FS) & Yes & JIJ2-3'NFI & & $\begin{array}{l}\text { Unpublished } \\
\text { data, Cardiff }\end{array}$ \\
\hline T536A & $\begin{array}{l}\text { Ex40: c.71 } 27 \text { 27258del } 132 \\
\text { [Exon } 40 \text { deletion?] }\end{array}$ & $\begin{array}{l}132 \mathrm{bp} \\
\text { deletion } \\
\text { (FS) }\end{array}$ & Yes & JIJ2-IVS38 & NIA & $\begin{array}{l}\text { Unpublished } \\
\text { data, Cardiff }\end{array}$ \\
\hline T541.2 & & & Yes & $\mathrm{EV} I 20-3^{\prime} \mathrm{NFI}$ & & \\
\hline T54I.4 & & & Yes & $\mathrm{EV} I 20-3^{\prime} \mathrm{NFI}$ & & \\
\hline T539 & 90kb Deletion & $\begin{array}{l}\text { Genomic } \\
\text { deletion }\end{array}$ & & & $\begin{array}{l}\text { Duplication: } \\
\text { Ex|9b-25 }\end{array}$ & $\begin{array}{l}\text { Unpublished } \\
\text { data, Cardiff }\end{array}$ \\
\hline
\end{tabular}


Table SI. Continued

\begin{tabular}{|c|c|c|c|c|c|c|c|c|c|}
\hline $\begin{array}{l}\text { Patient } \\
\text { ID }\end{array}$ & Germline mutation & $\begin{array}{l}\text { Type of } \\
\text { germline } \\
\text { mutation }\end{array}$ & LOH & LOH markers & $\begin{array}{l}\text { Predicted } \\
\text { extent of } \\
\text { LOH }\end{array}$ & $\begin{array}{l}\text { Evidence } \\
\text { for genomic } \\
\text { deletion? } \\
\text { MLPA/CGH } \\
\text { arrayCGH/ } \\
\text { FISH }\end{array}$ & $\begin{array}{l}\text { Probable } \\
\text { mechanism }\end{array}$ & $\begin{array}{l}\text { No. } \\
\text { samples } \\
\text { with } \\
\text { LOH }\end{array}$ & Reference \\
\hline
\end{tabular}

\section{PNFs}

$37 a$

Ex24: c.4268A > G; $\quad$ Missense $\quad$ Yes

p.Lys I 423Arg

$\mathrm{HHH} 202, \mathrm{E5}, \mathrm{II} 2 \mathrm{~b}$,

NFI

Genomic

$20 / 29$

10

Complete gene deletion

( $1.4 \mathrm{Mb})$

\begin{tabular}{|c|c|c|c|c|c|c|c|c|c|}
\hline $37 b$ & & & Yes & $\begin{array}{l}\text { IVS27, EVI20, IVS38, } \\
3^{\prime} \mathrm{NFI} \text { - I Probable gene } \\
\text { deletion }\end{array}$ & $\begin{array}{l}\text { NFI and } 3^{\prime} \\
\text { flanking } \\
\text { region }\end{array}$ & MLPA & $\begin{array}{l}\text { Genomic } \\
\text { deletion }\end{array}$ & $20 / 29$ & 10 \\
\hline PNF & p.Tyr2486IlefsXI5 & $\begin{array}{l}\text { deletion } \\
\text { (FS) }\end{array}$ & & $30 \%$ of cells & & & gene deletion & & data, Cardiff \\
\hline
\end{tabular}

$\begin{array}{lll}\text { T26I } & \text { Ex3: c.288 + I delG } & \text { I bp } \\ \text { PNF } & \text { deletion at } & \\ & \text { a splice site } & \end{array}$

\begin{tabular}{|c|c|c|c|c|c|c|c|c|c|}
\hline $605-1$ & Ex4a: c.289-2A > G & Splice site & Yes & $\begin{array}{l}\text { DI7S975, IVS27TG24.8, } \\
\text { IVS27TG28.4, } \\
\text { DI7SII66, DI7SI880, } \\
\text { DI7S907, DI7SI788, } \\
\text { DI7SI86I, DI7SI809, } \\
\text { DI7S668, DI7S928 }\end{array}$ & $\begin{array}{l}\mathrm{NFI} \text { and } \\
\text { flanking } \\
\text { regions }\end{array}$ & MLPA & $\begin{array}{l}\text { Mitotic } \\
\text { recombination }\end{array}$ & $13 / 43$ & II \\
\hline 47 I T4 I I & $\begin{array}{l}\text { Ex4a: } \\
\text { c. } 440 \_44 \text { IGC > AA; } \\
\text { p.Cys } 147 X\end{array}$ & Nonsense & $\begin{array}{l}\text { LOH IVS27, } \\
\text { IVS38, } \\
\text { 3'NFI-I }\end{array}$ & IVS38, 3'NFI-I & $\begin{array}{l}\text { NFI and } 3^{\prime} \\
\text { flanking } \\
\text { region }\end{array}$ & NIA & & $20 / 29$ & 10 \\
\hline $8 /$ T328 & Ex4b: c. $480-2 A>G$ & Splice site & $\begin{array}{l}\text { LOH: } \\
\text { IVS27, IVS38 }\end{array}$ & IVS27, IVS38 & $\begin{array}{l}\text { Intragenic } \\
\mathrm{NFI}\end{array}$ & MLPA & $\begin{array}{l}\text { Mitotic } \\
\text { recombination }\end{array}$ & $20 / 29$ & 10 \\
\hline $335-3$ & $\begin{array}{l}\text { Ex4b: c.528T >A; } \\
\text { p.Asp I76Glu }\end{array}$ & Missense & Yes & $\begin{array}{l}\text { DI7S2237, } \\
\text { IVS27TG24.8, } \\
\text { DI7SII66, DI7SI800 }\end{array}$ & NFI & MLPA & $\begin{array}{l}\text { Genomic } \\
\text { deletion }\end{array}$ & $13 / 43$ & II \\
\hline
\end{tabular}


Table SI. Continued

\begin{tabular}{|c|c|c|c|c|c|c|c|c|c|}
\hline $\begin{array}{l}\text { Patient } \\
\text { ID }\end{array}$ & Germline mutation & $\begin{array}{l}\text { Type of } \\
\text { germline } \\
\text { mutation }\end{array}$ & LOH & LOH markers & $\begin{array}{l}\text { Predicted } \\
\text { extent of } \\
\text { LOH }\end{array}$ & $\begin{array}{l}\text { Evidence } \\
\text { for genomic } \\
\text { deletion? } \\
\text { MLPA/CGH } \\
\text { arrayCGH/ } \\
\text { FISH }\end{array}$ & $\begin{array}{l}\text { Probable } \\
\text { mechanism }\end{array}$ & $\begin{array}{l}\text { No. } \\
\text { samples } \\
\text { with } \\
\text { LOH }\end{array}$ & Reference \\
\hline 59 & $\begin{array}{l}\text { Ex6: c. } 752 \text { dupA; } \\
\text { p.Asp24IGlufsX7 }\end{array}$ & $\begin{array}{l}\text { Small } \\
\text { Insertion } \\
\text { (fs) }\end{array}$ & Yes & intron 38 marker 53.0 & $\begin{array}{l}\text { Intragenic } \\
\mathrm{NFI}\end{array}$ & NIA & & $1 / 38$ & 12 \\
\hline T265.2 & $\begin{array}{l}\text { Ex9: c. I I86-I3delT } \\
\text { (Pathogenicity?) }\end{array}$ & $\begin{array}{l}\text { I bp } \\
\text { deletion } \\
\text { within a } \\
\text { splice site }\end{array}$ & $\begin{array}{l}\text { LOH ivs } 27, \\
\text { ivs } 38\end{array}$ & & & & & & \\
\hline $374-4$ & $\begin{array}{l}\text { ExI0a: c. } 1318 \mathrm{C}>\mathrm{T} \text {; } \\
\text { p.Arg440X }\end{array}$ & Nonsense & Yes & $\begin{array}{l}\text { IVS27TG24.8, } \\
\text { IVS27TG28.4, } \\
\text { DI7SII66, DI7SI880, } \\
\text { DI7S907, DI7SI86I }\end{array}$ & $\begin{array}{l}\mathrm{NFI} \text { and } \\
3^{\prime} \text { flanking } \\
\text { region }\end{array}$ & MLPA & $\begin{array}{l}\text { Mitotic } \\
\text { recombination }\end{array}$ & $13 / 43$ & II \\
\hline $\begin{array}{l}14 a / \\
T 4 \mid 2\end{array}$ & $\begin{array}{l}\text { ExI3: c. } 2076 C>\text { G; } \\
\text { p.Tyr692X }\end{array}$ & Nonsense & II 2B, 3' NFI & II $2 \mathrm{~B}, 3^{\prime} \mathrm{NFI}-\mathrm{I}$ & $\begin{array}{l}\mathrm{NFI} \text { and } \\
3^{\prime} \text { flanking } \\
\text { region }\end{array}$ & MLPA & $\begin{array}{l}\text { Mitotic } \\
\text { recombination }\end{array}$ & $20 / 29$ & 10 \\
\hline T263 & Ex|5: c.2326-2A > T & Splice site & $\begin{array}{l}\text { LOH ivs } 27 \\
\text { [rest hom] }\end{array}$ & & & & & & \\
\hline 22 / T394 & $\begin{array}{l}\text { Exl6: c. } 2446 C>T ; \\
\text { p.Arg816X }\end{array}$ & Nonsense & IVS27 & IVS 27, EVI20 & $\begin{array}{l}\text { Intragenic } \\
\mathrm{NFI}\end{array}$ & MLPA & $\begin{array}{l}\text { Mitotic } \\
\text { recombination }\end{array}$ & $20 / 29$ & 10 \\
\hline T437.2 & $\begin{array}{l}\text { ExI6: c.2497delT; } \\
\text { p.Ser833ProfsX7 }\end{array}$ & $\begin{array}{l}\text { I bp } \\
\text { deletion } \\
\text { (FS) }\end{array}$ & $\begin{array}{l}I-6 \mathrm{ex} \\
1,2,3,4 \mathrm{a}, 4 \mathrm{~b}, 4 \mathrm{c} \\
6 \text { deletion }\end{array}$ & & & NIA & & & \\
\hline T212 & $\begin{array}{l}\text { ExI6: c. } 2705 \text { deT; } \\
\text { p.Met } 902 \text { Argfs X22 }\end{array}$ & $\begin{array}{l}\text { I bp } \\
\text { deletion } \\
\text { (FS) }\end{array}$ & & & & & $\begin{array}{l}\text { ExI-Ex 4I } \\
\text { deletion } \\
\text { [variable ?] }\end{array}$ & & \\
\hline $18 / T 298$ & ExI8: c.31I3+IG >A & Splice site & $\begin{array}{l}\text { LOH } \\
: \mathrm{HHH} 202, \\
\text { IVS } 27\end{array}$ & IVS 27 & $\begin{array}{l}\text { Intragenic } \\
\mathrm{NFI}\end{array}$ & MLPA & Inconclusive & $20 / 29$ & 10 \\
\hline $30 / \mathrm{T} 342$ & $\begin{array}{l}\text { Ex|9a: c. } 3 \text { I } 23 \mathrm{G}>\mathrm{T} \\
\text { p.Met } 04 \mathrm{Ille}\end{array}$ & Missense & Yes & Determined by MLPA & $\begin{array}{l}\mathrm{NFI} \\
(\mathrm{I} .4 \mathrm{Mb})\end{array}$ & MLPA & $\begin{array}{l}\text { Genomic } \\
\text { deletion }\end{array}$ & $20 / 29$ & 10 \\
\hline
\end{tabular}


Table SI. Continued

\begin{tabular}{|c|c|c|c|c|c|c|c|c|c|}
\hline $\begin{array}{l}\text { Patient } \\
\text { ID }\end{array}$ & Germline mutation & $\begin{array}{l}\text { Type of } \\
\text { germline } \\
\text { mutation }\end{array}$ & LOH & LOH markers & $\begin{array}{l}\text { Predicted } \\
\text { extent of } \\
\text { LOH }\end{array}$ & $\begin{array}{l}\text { Evidence } \\
\text { for genomic } \\
\text { deletion? } \\
\text { MLPA/CGH } \\
\text { arrayCGH/ } \\
\text { FISH }\end{array}$ & $\begin{array}{l}\text { Probable } \\
\text { mechanism }\end{array}$ & $\begin{array}{l}\text { No. } \\
\text { samples } \\
\text { with } \\
\text { LOH }\end{array}$ & Reference \\
\hline 5 & $\begin{array}{l}\text { Ex20: } \\
\text { c.345\&_3460delCTCA; } \\
\text { p.Leul I 53MetfsX3 }\end{array}$ & $\begin{array}{l}4 \text { bp } \\
\text { deletion } \\
\text { (FS) }\end{array}$ & Yes & NFI gene & NFI & & & $1 / 3$ & 13 \\
\hline $\begin{array}{l}23 / \\
\text { T373.2 }\end{array}$ & $\begin{array}{l}\text { Ex22: c. } 3826 C>T ; \\
\text { p.Arg } 1276 X\end{array}$ & Nonsense & $\begin{array}{l}\text { WG deletion } \\
\text { [mixed cell } \\
\text { population] }\end{array}$ & IVS 27, IVS38 & $\begin{array}{l}\text { Intragenic } \\
\mathrm{NFI}\end{array}$ & MLPA & Inconclusive & $20 / 29$ & 10 \\
\hline $452 \mathrm{~T}$ & $\begin{array}{l}\text { Ex23.2: c. } 4084 C>T ; \\
\text { p.Argl } 362 X\end{array}$ & Nonsense & Yes & $\begin{array}{l}\text { NF-exon5 RFLP, NF- } \\
\text { (GATN)n intron 26, NF- } \\
\text { Alu(AAAT)n(i27b), NF- } \\
\text { EVI2B RFLP(i27b), NF- } \\
\text { EVI2A RFLP(i27b), NF- } \\
\text { IVSAC28.4(i27b), NF- } \\
\text { Evi-20, NF- } \\
\text { IVS38TG53.0, NF intron } \\
\text { 4I RFLP, DI7S57 } \\
\text { (EW206), DI7S250, } \\
\text { DI7SI30I, DI7S384 }\end{array}$ & $\begin{array}{l}\mathrm{NFI} \text { and } \\
3^{\prime} \text { flanking } \\
\text { region }\end{array}$ & NIA & & $4 / 10$ & 9 \\
\hline $27 / \mathrm{T} 30 \mathrm{I}$ & $\begin{array}{l}\text { Ex23.2: c. } 4095 \mathrm{C}>\mathrm{A} \text {; } \\
\text { p.Cys I } 365 \mathrm{X}\end{array}$ & Nonsense & Yes & Determined by MLPA & $\begin{array}{l}\text { Intragenic } \\
\mathrm{NFI}\end{array}$ & MLPA & $\begin{array}{l}\text { Genomic } \\
\text { deletion }\end{array}$ & $20 / 29$ & 10 \\
\hline T330 & $\begin{array}{l}\text { Ex24: c. } 4267 \mathrm{~A}>\mathrm{G} ; \\
\text { p.Lys I } 423 \mathrm{Glu}\end{array}$ & Missense & IVS27 & & & NIA & & & \\
\hline $\begin{array}{l}6 / \mathrm{T} 362 / \\
\text { T395 }\end{array}$ & $\begin{array}{l}\text { Ex24: c. } 4268 A>G ; \\
\text { p.Lys I 423Arg }\end{array}$ & Missense & Yes & EW206, EW207 & $\begin{array}{l}\text { Intragenic } \\
\mathrm{NFI}\end{array}$ & MLPA & Inconclusive & $20 / 29$ & \\
\hline $\begin{array}{l}\text { T362 } \\
\text { PNF }\end{array}$ & $\begin{array}{l}\text { Ex24: c. } 4268 A \text { > G; } \\
\text { p.Lys I } 423 \text { Arg }\end{array}$ & Missense & $\begin{array}{l}\text { LOH: } \\
\text { HHH202, E5, } \\
\text { II 2b, EVI20, } \\
\text { 3'NF }\end{array}$ & & & NIA & & & \\
\hline $\begin{array}{l}\text { T395 } \\
\text { PNF }\end{array}$ & $\begin{array}{l}\text { Ex24: c. } 4268 A \text { > G; } \\
\text { p.Lys I 423Arg }\end{array}$ & Missense & $\begin{array}{l}\text { LOH:IVS27, } \\
\text { EVI20, IVS38, } \\
\text { 3'NFI-I }\end{array}$ & & & & & & \\
\hline
\end{tabular}


Table SI. Continued

\begin{tabular}{|c|c|c|c|c|c|c|c|c|c|}
\hline $\begin{array}{l}\text { Patient } \\
\text { ID }\end{array}$ & Germline mutation & $\begin{array}{l}\text { Type of } \\
\text { germline } \\
\text { mutation }\end{array}$ & LOH & LOH markers & $\begin{array}{l}\text { Predicted } \\
\text { extent of } \\
\text { LOH }\end{array}$ & $\begin{array}{l}\text { Evidence } \\
\text { for genomic } \\
\text { deletion? } \\
\text { MLPA/CGH } \\
\text { arrayCGH/ } \\
\text { FISH }\end{array}$ & $\begin{array}{l}\text { Probable } \\
\text { mechanism }\end{array}$ & $\begin{array}{l}\text { No. } \\
\text { samples } \\
\text { with } \\
\text { LOH }\end{array}$ & Reference \\
\hline $317-1$ & Ex25: c. $4270-2 A>G$ & Splice site & Yes & $\begin{array}{l}\text { IVS27TG24.8, } \\
\text { IVS27TG28.4, } \\
\text { DI7SII I66 }\end{array}$ & NFI & MLPA & $\begin{array}{l}\text { Genomic } \\
\text { deletion }\end{array}$ & $13 / 43$ & 11 \\
\hline T393 & $\begin{array}{l}\text { Ex27a: c. } 4537 C>T \\
\text { p.Argl5। }\end{array}$ & Nonsense & & & & & $\begin{array}{l}\text { Whole gene } \\
\text { deletion }\end{array}$ & & \\
\hline $26 / T 300$ & $\begin{array}{l}\text { Ex29: } \\
\text { c.5227_5229delGTAinsT; } \\
\text { p.Val I743TyrfsXI7 }\end{array}$ & Indel (FS) & Yes & Determined by MLPA & $\begin{array}{l}\mathrm{NFI} \\
(1.4 \mathrm{Mb})\end{array}$ & MLPA & $\begin{array}{l}\text { Genomic } \\
\text { deletion }\end{array}$ & $20 / 29$ & 10 \\
\hline $338-2$ & $\begin{array}{l}\text { Ex29: c.5290delG; } \\
\text { p.Ala 1 764Leufs X8 }\end{array}$ & $\begin{array}{l}\text { I bp } \\
\text { deletion } \\
\text { (FS) }\end{array}$ & Yes & $\begin{array}{l}\text { DI7S783, DI7S975, } \\
\text { IVS27TG28.4, } \\
\text { DI7SII66, DI7SI880 }\end{array}$ & $\begin{array}{l}\mathrm{NFI} \text { and } \\
\text { flanking } \\
\text { regions }\end{array}$ & MLPA & $\begin{array}{l}\text { Genomic } \\
\text { deletion }\end{array}$ & $13 / 43$ & 11 \\
\hline $952-8$ & Ex30: c.5749+4delA & Splice site & Yes & $\begin{array}{l}\text { DI7S975, DI7SI880, } \\
\text { DI7S907, DI7SI788, } \\
\text { DI7SI86I, DI7SI809, } \\
\text { DI7S668, DI7S928 }\end{array}$ & $\begin{array}{l}\mathrm{NFI} \text { and } \\
\text { flanking } \\
\text { regions }\end{array}$ & MLPA & $\begin{array}{l}\text { Genomic } \\
\text { deletion }\end{array}$ & & \\
\hline 34 / T392 & $\begin{array}{l}\text { Ex31: } \\
\text { c.5750_5754dupGTATT; } \\
\text { p.Glu 1919Valfs X4 }\end{array}$ & $\begin{array}{l}5 \text { bp } \\
\text { duplication } \\
\text { (FS) }\end{array}$ & Yes & EVI20, IVS38, 3'NFI-I, & $\begin{array}{l}\mathrm{NFI} \text { and } \\
3^{\prime} \text { flanking } \\
\text { region }\end{array}$ & NIA & & $20 / 29$ & 10 \\
\hline $21 / T 357$ & $\begin{array}{l}\text { Ex37: c.679I dupA; } \\
\text { p.Tyr2264X }\end{array}$ & $\begin{array}{l}\text { I bp } \\
\text { duplication } \\
\text { (FS) }\end{array}$ & LOH : EW206 & EW206 & $\begin{array}{l}\text { Intragenic } \\
\mathrm{NFI}\end{array}$ & MLPA & $\begin{array}{l}\text { Mitotic } \\
\text { recombination }\end{array}$ & & \\
\hline T375 & $\begin{array}{l}\text { Ex40: c. } 7237 C>T ; \\
\text { p.Gln24I3X }\end{array}$ & Nonsense & $\begin{array}{l}\text { 16Ex } 16 \\
\text { deletion, } \\
\text { ex13 \& } 18 \\
\text { also lower }\end{array}$ & & & NIA & & & \\
\hline 7 & $\begin{array}{l}\text { Ex4l: c.7285C > T; } \\
\text { p.Arg2429X }\end{array}$ & Nonsense & Yes & $\mathrm{HHH} 202$, IVS27 & $\begin{array}{l}\text { Intragenic } \\
\mathrm{NFI}\end{array}$ & MLPA & $\begin{array}{l}\text { Mitotic } \\
\text { recombination }\end{array}$ & & \\
\hline
\end{tabular}


Table SI. Continued

\begin{tabular}{|c|c|c|c|c|c|c|c|c|c|}
\hline $\begin{array}{l}\text { Patient } \\
\text { ID }\end{array}$ & Germline mutation & $\begin{array}{l}\text { Type of } \\
\text { germline } \\
\text { mutation }\end{array}$ & LOH & LOH markers & $\begin{array}{l}\text { Predicted } \\
\text { extent of } \\
\text { LOH }\end{array}$ & $\begin{array}{l}\text { Evidence } \\
\text { for genomic } \\
\text { deletion? } \\
\text { MLPA/CGH } \\
\text { arrayCGH/ } \\
\text { FISH }\end{array}$ & $\begin{array}{l}\text { Probable } \\
\text { mechanism }\end{array}$ & $\begin{array}{l}\text { No. } \\
\text { samples } \\
\text { with } \\
\text { LOH }\end{array}$ & Reference \\
\hline I5 / T407 & $\begin{array}{l}\text { Ex46: c. } 7926 \text { dupT; } \\
\text { p.Lys } 2643 X\end{array}$ & $\begin{array}{l}\text { I bp } \\
\text { duplication } \\
\text { (FS) }\end{array}$ & $\begin{array}{l}\text { LOH 3'NFI-I, } \\
\text { EW206 }\end{array}$ & $3^{\prime} \mathrm{NFI}-\mathrm{I}$ & $\begin{array}{l}\mathrm{NFI} \text { and } \\
3^{\prime} \text { flanking } \\
\text { region }\end{array}$ & MLPA & Inconclusive & & \\
\hline $\begin{array}{l}\text { cl UK I } \\
\text { T56 }\end{array}$ & $\begin{array}{l}\text { Ex46: c.8035A > T; } \\
\text { p.Thr2679Ser }\end{array}$ & Missense & LOH IVS27 & IVS27 & $\begin{array}{l}\text { Intragenic } \\
\mathrm{NFI}\end{array}$ & NIA & & & \\
\hline T408 & Segmental NFI NI & $\mathrm{NI}$ & $\begin{array}{l}\text { LOH: IVS27, } \\
\text { IVS38, } \\
\text { 3'NFI-I }\end{array}$ & & & & & & \\
\hline T377 & Segmental NFI NI & $\mathrm{NI}$ & WG deletion & & & & & & \\
\hline 39 & Segmental NFI NI & $\mathrm{NI}$ & Yes & Determined by MLPA & $\begin{array}{l}\text { NFI } \\
(\mathrm{I} . \mathrm{I} \mathrm{Mb})\end{array}$ & MLPA & $\begin{array}{l}\text { Genomic } \\
\text { deletion }\end{array}$ & & \\
\hline 43 & Segmental NFI NI & $\mathrm{NI}$ & Yes & IVS27, IVS38, 3’NFI-I & $\begin{array}{l}\mathrm{NFI} \text { and } \\
3^{\prime} \text { flanking } \\
\text { region }\end{array}$ & MLPA & $\begin{array}{l}\text { Mitotic } \\
\text { recombination }\end{array}$ & & \\
\hline T385.I & $\mathrm{NI}$ & $\mathrm{NI}$ & Yes & & & NIA & & & $\begin{array}{l}\text { Unpublished } \\
\text { data, Cardiff }\end{array}$ \\
\hline T385.2 & $\mathrm{NI}$ & $\mathrm{NI}$ & $\mathrm{LOH} / \mathrm{del}$ & & & & & & \\
\hline T316 & $\mathrm{NI}$ & NI & Yes & $\begin{array}{l}\text { LOH: } \\
\text { HHH202, } \\
\text { E5, II 2b, } \\
\text { EVI20,3'NF, } \\
\text { C7I/2, EW206 }\end{array}$ & & & & & \\
\hline $76,45-95$ & $\mathrm{NI}$ & $\mathrm{NI}$ & Yes & $\begin{array}{l}\text { IVS27AC28.4, } \\
\text { IVS27TG24.8, } \\
\text { IVS38GT53 }\end{array}$ & $\begin{array}{l}\text { Intragenic } \\
\text { NFI }\end{array}$ & NIA & & $8 / 14$ & 14 \\
\hline$x I, 47-95$ & $\mathrm{NI}$ & $\mathrm{NI}$ & Yes & $\begin{array}{l}\text { IVS27AC28.4, M98509, } \\
\text { IVS38GT53 }\end{array}$ & $\begin{array}{l}\text { Intragenic } \\
\mathrm{NFI}\end{array}$ & & & & \\
\hline
\end{tabular}


Table SI. Continued

\begin{tabular}{|c|c|c|c|c|c|c|c|c|c|}
\hline $\begin{array}{l}\text { Patient } \\
\text { ID }\end{array}$ & Germline mutation & $\begin{array}{l}\text { Type of } \\
\text { germline } \\
\text { mutation }\end{array}$ & LOH & LOH markers & $\begin{array}{l}\text { Predicted } \\
\text { extent of } \\
\text { LOH }\end{array}$ & $\begin{array}{l}\text { Evidence } \\
\text { for genomic } \\
\text { deletion? } \\
\text { MLPA/CGH } \\
\text { arrayCGH/ } \\
\text { FISH }\end{array}$ & $\begin{array}{l}\text { Probable } \\
\text { mechanism }\end{array}$ & $\begin{array}{l}\text { No. } \\
\text { samples } \\
\text { with } \\
\text { LOH }\end{array}$ & Reference \\
\hline
\end{tabular}

\begin{tabular}{|c|c|c|c|c|c|c|c|c|}
\hline XI, 27-97 & $\mathrm{NI}$ & $\mathrm{NI}$ & Yes & $\begin{array}{l}\text { IVS27AC28.4, M98509, } \\
\text { IVS38GT53 }\end{array}$ & $\begin{array}{l}\text { Intragenic } \\
\text { NFI }\end{array}$ & & & \\
\hline $\begin{array}{l}293 \\
71-97\end{array}$ & $\mathrm{NI}$ & $\mathrm{NI}$ & Yes & $\begin{array}{l}\text { M98509, IVS27TG24.8, } \\
\text { IVS38GT53 }\end{array}$ & $\begin{array}{l}\text { Intragenic } \\
\text { NFI }\end{array}$ & & & \\
\hline $\begin{array}{l}293 \\
124-98\end{array}$ & $\mathrm{NI}$ & $\mathrm{NI}$ & Yes & $\begin{array}{l}\text { M98509, IVS27TG24.8, } \\
\text { IVS38GT54 }\end{array}$ & $\begin{array}{l}\text { Intragenic } \\
\text { NFI }\end{array}$ & & & \\
\hline $\begin{array}{l}290, \\
83-97\end{array}$ & $\mathrm{NI}$ & $\mathrm{NI}$ & Yes & $\begin{array}{l}\text { IVS27AC28.4, } \\
\text { IVS27TG24.8, } \\
\text { IVS38GT53 }\end{array}$ & $\begin{array}{l}\text { Intragenic } \\
\text { NFI }\end{array}$ & & & \\
\hline $\begin{array}{l}290 \\
121-98\end{array}$ & $\mathrm{NI}$ & $\mathrm{NI}$ & Yes & $\begin{array}{l}\text { IVS27AC28.4, } \\
\text { IVS27TG24.8, } \\
\text { IVS38GT53 }\end{array}$ & $\begin{array}{l}\text { Intragenic } \\
\text { NFI }\end{array}$ & & & \\
\hline $\begin{array}{l}292 \\
122-98\end{array}$ & $\mathrm{NI}$ & $\mathrm{NI}$ & Yes & $\begin{array}{l}\text { IVS27TG24.8, } \\
\text { IVS38GT53 }\end{array}$ & $\begin{array}{l}\text { Intragenic } \\
\text { NFI }\end{array}$ & & & \\
\hline PD-TI & $\mathrm{NI}$ & $\mathrm{NI}$ & Yes & $\begin{array}{l}\text { NF-Alu(AAAT)n(i27b), } \\
\text { DI7SI } 800\end{array}$ & $\begin{array}{l}\text { Intragenic } \\
\text { NFI }\end{array}$ & NIA & $4 / 10$ & 9 \\
\hline 386 T & $\mathrm{NI}$ & $\mathrm{NI}$ & & $\begin{array}{l}\text { NF-Alu(AAAT) } \\
\text { n(i27b), NF- } \\
\text { IVSAC28.4(i27b), } \\
\text { NF-Evi-20, NF- } \\
\text { IVS38TG53.0, } \\
\text { DI7SI800 }\end{array}$ & NFI & & & \\
\hline 454T-V & $\mathrm{NI}$ & $\mathrm{NI}$ & Yes & $\begin{array}{l}\text { NF-Alu(AAAT)n(i27b), } \\
\text { NF-EVI2B RFLP(i27b), } \\
\text { NF-IVSAC28.4(i27b), } \\
\text { NF-Evi-20, NF intron 4I } \\
\text { RFLP, DI7S57 } \\
\text { (EW206), DI7SI30I }\end{array}$ & NFI & & & \\
\hline
\end{tabular}


Table SI. Continued

\begin{tabular}{|c|c|c|c|c|c|c|c|c|c|}
\hline $\begin{array}{l}\text { Patient } \\
\text { ID }\end{array}$ & Germline mutation & $\begin{array}{l}\text { Type of } \\
\text { germline } \\
\text { mutation }\end{array}$ & LOH & LOH markers & $\begin{array}{l}\text { Predicted } \\
\text { extent of } \\
\text { LOH }\end{array}$ & $\begin{array}{l}\text { Evidence } \\
\text { for genomic } \\
\text { deletion? } \\
\text { MLPA/CGH } \\
\text { arrayCGH/ } \\
\text { FISH }\end{array}$ & $\begin{array}{l}\text { Probable } \\
\text { mechanism }\end{array}$ & $\begin{array}{l}\text { No. } \\
\text { samples } \\
\text { with } \\
\text { LOH }\end{array}$ & Reference \\
\hline NF284-I & $\mathrm{NI}$ & $\mathrm{NI}$ & Yes & $\begin{array}{l}\text { Exon } 28 \text { । } 4 \mathrm{bp} \\
\text { duplication marker } \\
\text { (specific } \\
\text { to germline } \\
\text { lesion found) }\end{array}$ & $\begin{array}{l}\text { Intragenic } \\
\text { NFI }\end{array}$ & & & $1 / 1$ & 8 \\
\hline 2654-97 & $\mathrm{NI}$ & $\mathrm{NI}$ & Yes & Determined by FISH & $\begin{array}{l}\text { Whole } \\
\text { chromosome }\end{array}$ & FISH & $\begin{array}{l}\text { Genomic } \\
\text { deletion }\end{array}$ & $1 / 11$ & 15 \\
\hline 385 & $\mathrm{NI}$ & $\mathrm{NI}$ & Yes & $\begin{array}{l}\text { DI7S975, DI7SI880, } \\
\text { DI7S907, DI7SI788, } \\
\text { DI7SI86I, DI7SI809, } \\
\text { DI7S668, DI7S928 }\end{array}$ & $\begin{array}{l}\mathrm{NFI} \text { and } \\
\text { flanking } \\
\text { regions }\end{array}$ & MLPA & $\begin{array}{l}\text { Mitotic } \\
\text { recombination }\end{array}$ & $13 / 43$ & 11 \\
\hline $389-2$ & $\mathrm{NI}$ & $\mathrm{NI}$ & Yes & $\begin{array}{l}\text { DI7S975, DI7SI307, } \\
\text { DI7S2237, } \\
\text { IVS27TG28.4, } \\
\text { DI7SI800, DI7SI880, } \\
\text { DI7S907, DI7SI86I, } \\
\text { DI7SI809, DI7S668, } \\
\text { DI7S928 }\end{array}$ & $\begin{array}{l}\mathrm{NFI} \text { and } \\
\text { flanking } \\
\text { regions }\end{array}$ & MLPA & $\begin{array}{l}\text { Mitotic } \\
\text { recombination }\end{array}$ & & \\
\hline 604-4 & $\mathrm{NI}$ & $\mathrm{NI}$ & Yes & $\begin{array}{l}\text { DI7SI800, DI7SI880, } \\
\text { DI7S907, DI7SI86I, } \\
\text { DI7S928 }\end{array}$ & $\begin{array}{l}\mathrm{NFI} \text { and } \\
\text { flanking } \\
\text { regions }\end{array}$ & MLPA & $\begin{array}{l}\text { Mitotic } \\
\text { recombination }\end{array}$ & & \\
\hline $913-5$ & $\mathrm{NI}$ & $\mathrm{NI}$ & Yes & $\begin{array}{l}\text { DI7S2237, } \\
\text { IVS27TG24.8, } \\
\text { DI7SI880, DI7SI788, } \\
\text { DI7SI86I }\end{array}$ & $\begin{array}{l}\text { NFI and } \\
3^{\prime} \text { flanking } \\
\text { region }\end{array}$ & MLPA & $\begin{array}{l}\text { Genomic } \\
\text { deletion }\end{array}$ & & \\
\hline $612-1$ & $\mathrm{NI}$ & $\mathrm{NI}$ & Yes & $\begin{array}{l}\text { DI7SI307, DI7S2237, } \\
\text { IVS27TG24.8, } \\
\text { DI7SII66, DI7S1800, } \\
\text { DI7SI880 }\end{array}$ & $\begin{array}{l}\mathrm{NFI} \text { and } \\
\text { flanking } \\
\text { regions }\end{array}$ & MLPA & $\begin{array}{l}\text { Genomic } \\
\text { deletion }\end{array}$ & & \\
\hline
\end{tabular}


Table SI. Continued

\begin{tabular}{|c|c|c|c|c|c|c|c|c|c|}
\hline $\begin{array}{l}\text { Patient } \\
\text { ID }\end{array}$ & Germline mutation & $\begin{array}{l}\text { Type of } \\
\text { germline } \\
\text { mutation }\end{array}$ & LOH & LOH markers & $\begin{array}{l}\text { Predicted } \\
\text { extent of } \\
\text { LOH }\end{array}$ & $\begin{array}{l}\text { Evidence } \\
\text { for genomic } \\
\text { deletion? } \\
\text { MLPA/CGH } \\
\text { arrayCGH/ } \\
\text { FISH }\end{array}$ & $\begin{array}{l}\text { Probable } \\
\text { mechanism }\end{array}$ & $\begin{array}{l}\text { No. } \\
\text { samples } \\
\text { with } \\
\text { LOH }\end{array}$ & Reference \\
\hline $337-5$ & $\mathrm{NI}$ & $\mathrm{NI}$ & Yes & $\begin{array}{l}\text { DI7SI307, DI7S2237, } \\
\text { IVS27TG24.8, } \\
\text { DI7SII66, DI7SI800 }\end{array}$ & $\mathrm{NFI}$ & MLPA & $\begin{array}{l}\text { Genomic } \\
\text { deletion }\end{array}$ & & \\
\hline 390 & $\mathrm{NI}$ & $\mathrm{NI}$ & Yes & $\begin{array}{l}\text { IVS27TG } 24.8, \\
\text { IVS27TG28.4, } \\
\text { DI7SII66, DI7S1800 }\end{array}$ & $\mathrm{NFI}$ & MLPA & $\begin{array}{l}\text { Genomic } \\
\text { deletion }\end{array}$ & & \\
\hline 49 & $\mathrm{NI}$ & $\mathrm{NI}$ & Yes & $\begin{array}{l}\mathrm{HHH} 202, \mathrm{E} 5,1 \mathrm{I} 2 \mathrm{~b}, \\
\mathrm{EVI} 20,3^{\prime} \mathrm{NFI}-\mathrm{I}, \mathrm{C} 7 \mathrm{I} / 2, \\
\text { EW206 }\end{array}$ & NFI & MLPA & Inconclusive & $20 / 29$ & 10 \\
\hline \multicolumn{10}{|c|}{ Spinal neurofibromas } \\
\hline I & $\begin{array}{l}\text { Ex7: c.899T > C; } \\
\text { p.Leu300Pro }\end{array}$ & Missense & Yes & EVI20, IVS38 & $\begin{array}{l}\text { Intragenic } \\
\mathrm{NFI}\end{array}$ & MLPA & $\begin{array}{l}\text { Mitotic } \\
\text { recombination }\end{array}$ & $8 / 22$ & 16 \\
\hline 7 & $\begin{array}{l}\text { Ex9: c.I I 86-13delT } \\
\text { (Pathogenicity?) }\end{array}$ & $\begin{array}{l}\text { I bp } \\
\text { deletion } \\
\text { (FS) }\end{array}$ & Yes & IVS27, IVS38 & $\begin{array}{l}\text { Intragenic } \\
\mathrm{NFI}\end{array}$ & MLPA & $\begin{array}{l}\text { Mitotic } \\
\text { recombination }\end{array}$ & & \\
\hline 3 & Ex|6: c.24I0-2A >T & Splice site & Yes & IVS27 & $\begin{array}{l}\text { Intragenic } \\
\mathrm{NFI}\end{array}$ & MLPA & $\begin{array}{l}\text { Mitotic } \\
\text { recombination }\end{array}$ & & \\
\hline II.I & $\begin{array}{l}\text { Ex22: c.3827G > A; } \\
\text { p.Arg I 276Glu }\end{array}$ & Missense & Yes & IVS38 & $\begin{array}{l}\text { Intragenic } \\
\mathrm{NFI}\end{array}$ & MLPA & $\begin{array}{l}\text { Mitotic } \\
\text { recombination }\end{array}$ & & \\
\hline 11.2 & & & Yes & $\begin{array}{l}\text { Deletion of exons } \\
13>16\end{array}$ & $\begin{array}{l}\text { Intragenic } \\
\mathrm{NFI}\end{array}$ & MLPA & Deletion & & \\
\hline 2 & $\begin{array}{l}\text { Ex23.2: c. } 4066 \mathrm{G}>\mathrm{A} \text {; } \\
\text { p.Glu 1356Lys }\end{array}$ & Missense & Yes & 27, $3^{\prime} \mathrm{NFI}$ & $\mathrm{NFI}$ & MLPA & $\begin{array}{l}\text { Mitotic } \\
\text { recombination }\end{array}$ & & \\
\hline 10 & $\begin{array}{l}\text { Ex29: c.5242C > T; } \\
\text { p.Arg I } 748 X\end{array}$ & Nonsense & Yes & $\begin{array}{l}\text { II } 2 B \text {, AluI, JIJ2 and } \\
\text { EVI20 }\end{array}$ & $\begin{array}{l}\text { Intragenic } \\
\mathrm{NFI}\end{array}$ & MLPA & $\begin{array}{l}\text { Mitotic } \\
\text { recombination }\end{array}$ & & \\
\hline
\end{tabular}


Table SI. Continued

\begin{tabular}{|c|c|c|c|c|c|c|c|c|c|}
\hline $\begin{array}{l}\text { Patient } \\
\text { ID }\end{array}$ & Germline mutation & $\begin{array}{l}\text { Type of } \\
\text { germline } \\
\text { mutation }\end{array}$ & LOH & LOH markers & $\begin{array}{l}\text { Predicted } \\
\text { extent of } \\
\text { LOH }\end{array}$ & $\begin{array}{l}\text { Evidence } \\
\text { for genomic } \\
\text { deletion? } \\
\text { MLPA/CGH } \\
\text { arrayCGH/ } \\
\text { FISH }\end{array}$ & $\begin{array}{l}\text { Probable } \\
\text { mechanism }\end{array}$ & $\begin{array}{l}\text { No. } \\
\text { samples } \\
\text { with } \\
\text { LOH }\end{array}$ & Reference \\
\hline \multicolumn{10}{|c|}{ MPNSTs } \\
\hline T196.20 & Deletion exons 2 and 3 & $\begin{array}{l}\text { Two exon } \\
\text { deletion }\end{array}$ & Yes & $\begin{array}{l}\text { II 2b, IVS27AC28.4, } \\
\text { EVI20(IVS27B), } \\
\text { IVS38GT53.0 (IVS38), } \\
3^{\prime}-\text { NFI, C7/CTI/2 } \\
\text { (3'-UTR), EW206 } \\
\text { (3'extragenic), EW207 } \\
\text { (3'extragenic), DI7S798 }\end{array}$ & $\begin{array}{l}\mathrm{NFI} \text { and } \\
3^{\prime} \text { flanking } \\
\text { region }\end{array}$ & MLPA & $\begin{array}{l}\text { Mitotic } \\
\text { recombination }\end{array}$ & $2 / 11$ & 1 \\
\hline T196.24 & & & Yes & $\begin{array}{l}\text { NFI exon 5, II } 2 \mathrm{~b}, \\
\text { IVS27AC28.4, } \\
\text { EVI20(IVS27B), } \\
\text { IVS38GT53.0 (IVS38), } \\
3^{\prime}-\text { NFI, C7/CTI/2 } \\
\text { (3'-UTR), EW206 } \\
\text { (3'extragenic), EW207 } \\
\text { (3'extragenic) }\end{array}$ & $\begin{array}{l}\mathrm{NFI} \text { and } \\
3^{\prime} \text { flanking } \\
\text { region }\end{array}$ & MLPA & $\begin{array}{l}\text { Genomic } \\
\text { deletion }\end{array}$ & $2 / 11$ & 1 \\
\hline 13 & Deletion exons 2 and 3 & $\begin{array}{l}\text { Two exon } \\
\text { deletion }\end{array}$ & Yes & $\begin{array}{l}\text { Ex5, II 2b, IVS27, EVI20, } \\
\text { IVS38,C7CT, EW206, } \\
\text { EW207,3'NFI }\end{array}$ & $\begin{array}{l}\mathrm{NFI} \text { and } \\
3^{\prime} \text { flanking } \\
\text { region }\end{array}$ & $\begin{array}{l}\text { MLPA/CGH } \\
\text { array }\end{array}$ & $\begin{array}{l}\text { Genomic } \\
\text { deletion }\end{array}$ & $31 / 34$ & 17 \\
\hline 7 & Ex4c: c.654 + IG $>T$ & Splice site & Yes & $\begin{array}{l}\text { UTI72, HH2O2, JI/J2, } \\
\text { EVI20 }\end{array}$ & NFI & $\begin{array}{l}\text { MLPA/CGH } \\
\text { array }\end{array}$ & $\begin{array}{l}\text { Genomic } \\
\text { deletion }\end{array}$ & & \\
\hline 27 & $\begin{array}{l}\text { Ex8: } \\
\text { c. I I33_I I 36delACTG; } \\
\text { p.Asp378AlafsX7 }\end{array}$ & $\begin{array}{l}4 \text { bp } \\
\text { deletion } \\
\text { (FS) }\end{array}$ & Yes & Ex5, JIJ2,3'NFI & $\begin{array}{l}\text { NFI and } \\
3^{\prime} \text { flanking } \\
\text { region }\end{array}$ & NIA & & & \\
\hline 9 & $\begin{array}{l}\text { ExII: c. I7I3G > A; } \\
\text { p.Trp57IX }\end{array}$ & Nonsense & Yes & DI7SI82, II bb, JI//2 & $\begin{array}{l}\text { Intragenic } \\
\mathrm{NFI}\end{array}$ & & & & \\
\hline 10 & & & Yes & $\begin{array}{l}\text { UTI72, HH202, JI/J2, } \\
\text { EVI20, > 2.2Mb }\end{array}$ & NFI & $\begin{array}{l}\text { MLPA / CGH } \\
\text { array }\end{array}$ & $\begin{array}{l}\text { Genomic } \\
\text { deletion }\end{array}$ & & \\
\hline
\end{tabular}




\begin{tabular}{|c|c|c|c|c|c|c|c|c|c|}
\hline $\begin{array}{l}\text { Patient } \\
\text { ID }\end{array}$ & Germline mutation & $\begin{array}{l}\text { Type of } \\
\text { germline } \\
\text { mutation }\end{array}$ & LOH & LOH markers & $\begin{array}{l}\text { Predicted } \\
\text { extent of } \\
\text { LOH }\end{array}$ & $\begin{array}{l}\text { Evidence } \\
\text { for genomic } \\
\text { deletion? } \\
\text { MLPA/CGH } \\
\text { arrayCGH/ } \\
\text { FISH }\end{array}$ & $\begin{array}{l}\text { Probable } \\
\text { mechanism }\end{array}$ & $\begin{array}{l}\text { No. } \\
\text { samples } \\
\text { with } \\
\text { LOH }\end{array}$ & Reference \\
\hline 23 & $\begin{array}{l}\text { Ex|2a: c. } 1318 \mathrm{C}>\mathrm{T} \text {; } \\
\text { p.Arg } 440 \mathrm{X}\end{array}$ & Nonsense & Yes & HHH202, EVI20. IVS38 & $\begin{array}{l}\text { Intragenic } \\
\mathrm{NFI}\end{array}$ & NIA & & & \\
\hline 14 & $\begin{array}{l}\text { ExI2a: } \\
\text { c. } 1754 \text { I757delTAAC; } \\
\text { p.Thr586Valfs X19 }\end{array}$ & $\begin{array}{l}4 \text { bp } \\
\text { deletion } \\
\text { (FS) }\end{array}$ & Yes & IVS27, $3^{\prime} \mathrm{NFI}$ & $\begin{array}{l}\mathrm{NFI} \text { and } \\
3^{\prime} \text { flanking } \\
\text { region }\end{array}$ & $\begin{array}{l}\text { MLPA/CGH } \\
\text { array }\end{array}$ & $\begin{array}{l}\text { Mitotic } \\
\text { recombination }\end{array}$ & & \\
\hline 12 & Ex|3: c.2002-14C > G & Splice site & Yes & $\begin{array}{l}\text { II 2b, IVS27, EVI20, } \\
\text { IVS38, 3'NF }\end{array}$ & $\begin{array}{l}\mathrm{NFI} \text { and } \\
\text { 3' flanking } \\
\text { region }\end{array}$ & $\begin{array}{l}\text { MLPA/CGH } \\
\text { array }\end{array}$ & $\begin{array}{l}\text { Genomic } \\
\text { deletion }\end{array}$ & & \\
\hline 43 & $\begin{array}{l}\text { Ex|3: c.204IC > T; } \\
\text { p.Arg68IX }\end{array}$ & Nonsense & Yes & Determined by MLPA & NFI & MLPA & $\begin{array}{l}\text { Duplication } \\
\text { mitotic } \\
\text { recombination }\end{array}$ & $6 / 25$ & 18 \\
\hline 15 & $\begin{array}{l}\text { Ex16: c.2497delT; } \\
\text { p.Ser833Profs X7 }\end{array}$ & $\begin{array}{l}\text { I bp } \\
\text { deletion } \\
\text { (FS) }\end{array}$ & & $\begin{array}{l}\text { Intragenic Deletion } \\
\text { (Exons I-6) MLPA }\end{array}$ & $\begin{array}{l}\text { Intragenic } \\
\mathrm{NFI}\end{array}$ & $\begin{array}{l}\text { MLPA/CGH } \\
\text { array }\end{array}$ & $\begin{array}{l}\text { Genomic } \\
\text { deletion }\end{array}$ & $31 / 34$ & 17 \\
\hline 25 & $\begin{array}{l}\text { Ex16: c.2705delT; } \\
\text { p.Met902Argfs X22 }\end{array}$ & $\begin{array}{l}\text { I bp } \\
\text { deletion } \\
\text { (FS) }\end{array}$ & Yes & $\begin{array}{l}\text { Intragenic deletion } \\
\text { (exonsI-4I) MLPA }\end{array}$ & $\begin{array}{l}\text { Intragenic } \\
\mathrm{NFI}\end{array}$ & $\begin{array}{l}\text { MLPA/CGH } \\
\text { array }\end{array}$ & $\begin{array}{l}\text { Genomic } \\
\text { deletion }\end{array}$ & & \\
\hline 17 & $\begin{array}{l}\text { Ex20: } \\
\text { c.3457_3460delCTCA; } \\
\text { p.Leul I53MetfsX3 }\end{array}$ & $\begin{array}{l}4 \text { bp } \\
\text { deletion } \\
\text { (FS) }\end{array}$ & & & & NIA & & & \\
\hline 18 & & & Yes & $3^{\prime} \mathrm{NFI}$ & $\begin{array}{l}\text { Intragenic } \\
\mathrm{NFI}\end{array}$ & $\begin{array}{l}\text { MLPA/CGH } \\
\text { array }\end{array}$ & $\begin{array}{l}\text { Genomic } \\
\text { deletion }\end{array}$ & & \\
\hline 459T I & $\begin{array}{l}\text { Ex2I: c.3684delC; } \\
\text { p.Asn I229MetfsXII }\end{array}$ & $\begin{array}{l}\text { I bp } \\
\text { deletion } \\
\text { (FS) }\end{array}$ & Yes & $\begin{array}{l}\text { TP53(INTRONI), } \\
\text { TP53(INTRON6), NF- } \\
\text { (GATN)n INTRON26, } \\
\text { NF-IVSAC28.4(i27b), } \\
\text { DI7S57, DI7S250, } \\
\text { DI7SI 30I, DI7S784 }\end{array}$ & $\begin{array}{l}\text { Whole } \\
\text { chromosome }\end{array}$ & NIA & & $3 / 5$ & 9 \\
\hline
\end{tabular}


Table SI. Continued

\begin{tabular}{|c|c|c|c|c|c|c|c|c|c|}
\hline $\begin{array}{l}\text { Patient } \\
\text { ID }\end{array}$ & Germline mutation & $\begin{array}{l}\text { Type of } \\
\text { germline } \\
\text { mutation }\end{array}$ & LOH & LOH markers & $\begin{array}{l}\text { Predicted } \\
\text { extent of } \\
\text { LOH }\end{array}$ & $\begin{array}{l}\text { Evidence } \\
\text { for genomic } \\
\text { deletion? } \\
\text { MLPA/CGH } \\
\text { arrayCGH/ } \\
\text { FISH }\end{array}$ & $\begin{array}{l}\text { Probable } \\
\text { mechanism }\end{array}$ & $\begin{array}{l}\text { No. } \\
\text { samples } \\
\text { with } \\
\text { LOH }\end{array}$ & Reference \\
\hline 8 & $\begin{array}{l}\text { Ex22: c.3732delT; } \\
\text { p.Thr I 245Leufsx2 I }\end{array}$ & $\begin{array}{l}\text { I bp } \\
\text { deletion } \\
\text { (FS) }\end{array}$ & Yes & $\operatorname{lnt} 12$, JIJ2 & $\begin{array}{l}\text { Intragenic } \\
\text { NFI }\end{array}$ & & & $31 / 34$ & 17 \\
\hline 64 & Ex23.I: c.3368 + IdelG & $\begin{array}{l}\text { I bp } \\
\text { deletion at } \\
\text { a splice site }\end{array}$ & Yes & Determined by MLPA & NFI & MLPA & $\begin{array}{l}\text { Genomic } \\
\text { deletion }\end{array}$ & $6 / 25$ & 18 \\
\hline 56 & $\begin{array}{l}\text { Ex25: c. } 4276 C>A ; \\
\text { p.Gln I } 426 \text { Lys }\end{array}$ & Missense & Yes & Determined by MLPA & NFI & MLPA & $\begin{array}{l}\text { Duplication } \\
\text { mitotic } \\
\text { recombination }\end{array}$ & & \\
\hline 4 & $\begin{array}{l}\text { Ex27a: c. } 4537 C>T ; \\
\text { p.Arg।5।3X }\end{array}$ & Nonsense & Yes & IVS27b & $\begin{array}{l}\text { Intragenic } \\
\mathrm{NFI}\end{array}$ & $\begin{array}{l}\text { MLPA/CGH } \\
\text { array }\end{array}$ & $\begin{array}{l}\text { Genomic } \\
\text { deletion }\end{array}$ & $31 / 34$ & 17 \\
\hline 6 & $\begin{array}{l}\text { Ex28: c.5003insTG; } \\
\text { p.Tyr I668LeufsX7 }\end{array}$ & $\begin{array}{l}2 \text { bp } \\
\text { insertion } \\
\text { (FS) }\end{array}$ & Yes & I4b, JIJ2, EVI20 & NFI & $\begin{array}{l}\text { MLPA/CGH } \\
\text { array }\end{array}$ & $\begin{array}{l}\text { Genomic } \\
\text { deletion }\end{array}$ & & \\
\hline 21 & $\begin{array}{l}\text { Ex29: c.5234C > G; } \\
\text { p.Ser I 745X }\end{array}$ & Nonsense & Yes & Partial gene deletion & $\begin{array}{l}\text { Intragenic } \\
\text { NFI }\end{array}$ & $\begin{array}{l}\text { MLPA/CGH } \\
\text { array }\end{array}$ & $\begin{array}{l}\text { Genomic } \\
\text { deletion }\end{array}$ & & \\
\hline 19 & $\begin{array}{l}\text { Ex37: c. } 6792 C>A \text {; } \\
\text { p.Tyr2264X }\end{array}$ & Nonsense & Yes & $\begin{array}{l}\text { II 2b, IVS27, JIJ2, EVI20, } \\
\text { IVS38, C7CT }\end{array}$ & NFI & $\begin{array}{l}\text { MLPA/CGH } \\
\text { array }\end{array}$ & $\begin{array}{l}\text { Genomic } \\
\text { deletion }\end{array}$ & & \\
\hline 24 & $\begin{array}{l}\text { Ex38: c. } 696 \text { I insC; } \\
\text { p.Leu232I Profs X5 }\end{array}$ & $\begin{array}{l}\text { I bp } \\
\text { duplication } \\
\text { (FS) }\end{array}$ & Yes & Determined by MLPA & NFI & MLPA & $\begin{array}{l}\text { Genomic } \\
\text { deletion }\end{array}$ & $6 / 25$ & 18 \\
\hline I & $\begin{array}{l}\text { Ex4I: } \\
\text { c.7268_7269delCA; } \\
\text { p.Thr2423SerfsX2 }\end{array}$ & $\begin{array}{l}2 \text { bp } \\
\text { deletion } \\
\text { (FS) }\end{array}$ & Yes & Intron 4I-30 & $\begin{array}{l}\text { Intragenic } \\
\mathrm{NFI}\end{array}$ & $\begin{array}{l}\text { MLPA/CGH } \\
\text { array }\end{array}$ & $\begin{array}{l}\text { Genomic } \\
\text { deletion }\end{array}$ & $31 / 34$ & 17 \\
\hline 58 & $\mathrm{NI}$ & $\mathrm{NI}$ & Yes & HHH202, NFI, EW206 & $\begin{array}{l}\text { Intragenic } \\
\mathrm{NFI}\end{array}$ & & $\begin{array}{l}\text { Genomic } \\
\text { deletion }\end{array}$ & $6 / 11$ & 19 \\
\hline
\end{tabular}




\begin{tabular}{|c|c|c|c|c|c|c|c|c|c|}
\hline \multicolumn{10}{|c|}{ Table SI. Continued } \\
\hline $\begin{array}{l}\text { Patient } \\
\text { ID }\end{array}$ & Germline mutation & $\begin{array}{l}\text { Type of } \\
\text { germline } \\
\text { mutation }\end{array}$ & LOH & LOH markers & $\begin{array}{l}\text { Predicted } \\
\text { extent of } \\
\text { LOH }\end{array}$ & $\begin{array}{l}\text { Evidence } \\
\text { for genomic } \\
\text { deletion? } \\
\text { MLPA/CGH } \\
\text { arrayCGH/ } \\
\text { FISH }\end{array}$ & $\begin{array}{l}\text { Probable } \\
\text { mechanism }\end{array}$ & $\begin{array}{l}\text { No. } \\
\text { samples } \\
\text { with } \\
\text { LOH }\end{array}$ & Reference \\
\hline 52 & $\mathrm{NI}$ & $\mathrm{NI}$ & Yes & $\begin{array}{l}\text { HHH202, NFI, EW206, } \\
\text { EW207 }\end{array}$ & $\begin{array}{l}\text { Intragenic } \\
\mathrm{NFI}\end{array}$ & & $\begin{array}{l}\text { Genomic } \\
\text { deletion }\end{array}$ & & \\
\hline 22 & $\mathrm{NI}$ & $\mathrm{NI}$ & Yes & $\mathrm{HHH} 202$ & $\begin{array}{l}\text { Intragenic } \\
\mathrm{NFI}\end{array}$ & & $\begin{array}{l}\text { Genomic } \\
\text { deletion }\end{array}$ & & \\
\hline 8 & $\mathrm{NI}$ & $\mathrm{NI}$ & Yes & EW206, EW207 & $\begin{array}{l}\text { Intragenic } \\
\mathrm{NFI}\end{array}$ & & $\begin{array}{l}\text { Genomic } \\
\text { deletion }\end{array}$ & & \\
\hline 2 & $\mathrm{NI}$ & $\mathrm{NI}$ & Yes & $\begin{array}{l}\text { pl44D6, pYNZ22.I, } \\
\text { pYNH37.3, EW503 }\end{array}$ & $\begin{array}{l}\text { NFI region } \\
\text { and some } \\
17 \mathrm{p}\end{array}$ & & $\begin{array}{l}\text { Genomic } \\
\text { deletion }\end{array}$ & $5 / 6$ & 20 \\
\hline 3 & $\mathrm{NI}$ & $\mathrm{NI}$ & Yes & $\begin{array}{l}\text { EW503, EW30I (B), } \\
\text { EW30I (T) }\end{array}$ & $\begin{array}{l}\text { Intragenic } \\
\mathrm{NFI}\end{array}$ & & $\begin{array}{l}\text { Genomic } \\
\text { deletion }\end{array}$ & & \\
\hline 4 & $\mathrm{NI}$ & $\mathrm{NI}$ & Yes & $\begin{array}{l}\text { pl44D6, pYNZ22.I, } \\
\text { pYNH37.3, EW503, } \\
\text { EW30I (T), pHHH202, } \\
\text { EW207 (B), pTHH59 }\end{array}$ & $\begin{array}{l}\text { Whole } \\
\text { chromosome }\end{array}$ & & $\begin{array}{l}\text { Genomic } \\
\text { deletion }\end{array}$ & & \\
\hline 5 & $\mathrm{NI}$ & $\mathrm{NI}$ & Yes & $\begin{array}{l}\text { pI44D6, pYNZ22.I, } \\
\text { pYNH37.3, EW503, } \\
\text { EW30I (B), EW30I (T), } \\
\text { pHHH202, EW207 } \\
\text { (B) }\end{array}$ & $\begin{array}{l}\text { Whole } \\
\text { chromosome }\end{array}$ & & $\begin{array}{l}\text { Genomic } \\
\text { deletion }\end{array}$ & & \\
\hline 10 & $\mathrm{NI}$ & $\mathrm{NI}$ & Yes & pl44D6, pYNZ22.I, & $\begin{array}{l}\text { NFI region } \\
\text { and some } \\
17 \mathrm{p}\end{array}$ & & $\begin{array}{l}\text { Genomic } \\
\text { deletion }\end{array}$ & & \\
\hline $88-3 / 14$ & $\mathrm{NI}$ & $\mathrm{NI}$ & Yes & $\begin{array}{l}\text { DI7S30, TP53, DI7S7I, } \\
\text { DI7S8, DI7S57 }\end{array}$ & $\begin{array}{l}\text { Whole } \\
\text { chromosome }\end{array}$ & $\begin{array}{l}\text { G-banded } \\
\text { chromosome } \\
17 \\
\text { duplication }\end{array}$ & $\begin{array}{l}\text { Genomic } \\
\text { duplication }\end{array}$ & $3 / 9$ & 21 \\
\hline
\end{tabular}


Table SI. Continued

\begin{tabular}{|c|c|c|c|c|c|c|c|c|c|}
\hline $\begin{array}{l}\text { Patient } \\
\text { ID }\end{array}$ & Germline mutation & $\begin{array}{l}\text { Type of } \\
\text { germline } \\
\text { mutation }\end{array}$ & LOH & LOH markers & $\begin{array}{l}\text { Predicted } \\
\text { extent of } \\
\text { LOH }\end{array}$ & $\begin{array}{l}\text { Evidence } \\
\text { for genomic } \\
\text { deletion? } \\
\text { MLPA/CGH } \\
\text { arrayCGH/ } \\
\text { FISH }\end{array}$ & $\begin{array}{l}\text { Probable } \\
\text { mechanism }\end{array}$ & $\begin{array}{l}\text { No. } \\
\text { samples } \\
\text { with } \\
\text { LOH }\end{array}$ & Reference \\
\hline 88-8 & $\mathrm{NI}$ & $\mathrm{NI}$ & Yes & DI7S30, DI7S7I & NFI & NIA & & & \\
\hline $88-18$ & $\mathrm{NI}$ & $\mathrm{NI}$ & Yes & $\begin{array}{l}\text { DI7S30, DI7S7I, } \\
\text { DI7S2I, DI7S33, } \\
\text { EVI2B, DI7S82 }\end{array}$ & $\begin{array}{l}\text { Whole } \\
\text { chromosome }\end{array}$ & & & & \\
\hline I & $\mathrm{NI}$ & $\mathrm{NI}$ & Yes & $\begin{array}{l}\text { DI7S5, DI7SI, DI7SI37, } \\
\text { CRYBI, NFI, DI7SI } 46\end{array}$ & $\begin{array}{l}\mathrm{NFI} \text { and } \\
\text { flanking } \\
\text { regions }\end{array}$ & NIA & & $2 / 5$ & 22 \\
\hline 4 & $\mathrm{NI}$ & $\mathrm{NI}$ & Yes & DI7S34, DI7S5, DI7SI46 & $\begin{array}{l}\mathrm{NFI} \text { and } \\
\text { flanking } \\
\text { regions }\end{array}$ & & & & \\
\hline I & $\mathrm{NI}$ & $\mathrm{NI}$ & Yes & & NFI & & $\begin{array}{l}\text { Genomic } \\
\text { deletion }\end{array}$ & $1 / 1$ & 23 \\
\hline $\mathbf{I}$ & $\mathrm{NI}$ & $\mathrm{NI}$ & Yes & NFI alu, TP53 BHP53 & $\begin{array}{l}\text { Whole } \\
\text { chromosome }\end{array}$ & NIA & & $3 / 7$ & 24 \\
\hline 7 & $\mathrm{NI}$ & $\mathrm{NI}$ & Yes & $\begin{array}{l}\text { CRYBI, NFI alu, TP53 } \\
\text { BHP53 }\end{array}$ & $\begin{array}{l}\text { Whole } \\
\text { chromosome }\end{array}$ & & & & \\
\hline 8 & $\mathrm{NI}$ & $\mathrm{NI}$ & Yes & $\begin{array}{l}\text { DI7S4, DI7S74, NFI } \\
\text { e.3I, NFI alu }\end{array}$ & NFI & & & & \\
\hline $44 I T$ & $\mathrm{NI}$ & $\mathrm{NI}$ & Yes & $\begin{array}{l}\text { TP53(INTRON6), } \\
\text { DI7SI863, } \\
\text { DI7twbch=S33, NF- } \\
\text { IVSAC28.4(i27b), } \\
\text { NF-Evi-20, } \\
\text { NF-IVS38TG53.0, } \\
\text { DI7SI800, DI7S73, } \\
\text { DI7SI30I }\end{array}$ & $\begin{array}{l}\text { Whole } \\
\text { chromosome }\end{array}$ & NIA & & $3 / 5$ & 9 \\
\hline
\end{tabular}


Table SI. Continued

\begin{tabular}{|c|c|c|c|c|c|c|c|c|c|}
\hline $\begin{array}{l}\text { Patient } \\
\text { ID }\end{array}$ & Germline mutation & $\begin{array}{l}\text { Type of } \\
\text { germline } \\
\text { mutation }\end{array}$ & LOH & LOH markers & $\begin{array}{l}\text { Predicted } \\
\text { extent of } \\
\text { LOH }\end{array}$ & $\begin{array}{l}\text { Evidence } \\
\text { for genomic } \\
\text { deletion? } \\
\text { MLPA/CGH } \\
\text { arrayCGH/ } \\
\text { FISH }\end{array}$ & $\begin{array}{l}\text { Probable } \\
\text { mechanism }\end{array}$ & $\begin{array}{l}\text { No. } \\
\text { samples } \\
\text { with } \\
\text { LOH }\end{array}$ & Reference \\
\hline 396T4 & $\mathrm{NI}$ & $\mathrm{NI}$ & Yes & $\begin{array}{l}\text { NF-IVSAC28.4(i27b, } \\
\text { NF-IVS38TG53.0, } \\
\text { DI7S57, DI7S250, } \\
\text { DI7SI30I }\end{array}$ & $\begin{array}{l}\mathrm{NFI} \text { and } 3^{\prime} \\
\text { flanking } \\
\text { region }\end{array}$ & & & & \\
\hline 2 & $\mathrm{NI}$ & $\mathrm{NI}$ & Yes & NFI, PI6, TP53 & $\begin{array}{l}\text { Whole } \\
\text { chromosome }\end{array}$ & NIA & & $5 / 8$ & 13 \\
\hline $5 \mathbf{a}$ & $\mathrm{NI}$ & $\mathrm{NI}$ & Yes & NFI, PI6, TP53 & $\begin{array}{l}\text { Whole } \\
\text { chromosome }\end{array}$ & & & & \\
\hline $5 b$ & $\mathrm{NI}$ & $\mathrm{NI}$ & Yes & NFI, PI6, TP53 & $\begin{array}{l}\text { Whole } \\
\text { chromosome }\end{array}$ & & & & \\
\hline $6 \mathbf{a}$ & $\mathrm{NI}$ & $\mathrm{NI}$ & Yes & NFI, PI6, TP53 & $\begin{array}{l}\text { Whole } \\
\text { chromosome }\end{array}$ & & & & \\
\hline 6b & $\mathrm{NI}$ & $\mathrm{NI}$ & Yes & NFI, PI6, TP53 & $\begin{array}{l}\text { Whole } \\
\text { chromosome }\end{array}$ & & & & \\
\hline 2 & $\mathrm{NI}$ & $\mathrm{NI}$ & Yes & Total gene deletion & NFI & $\begin{array}{l}\text { MLPA/CGH } \\
\text { array }\end{array}$ & $\begin{array}{l}\text { Genomic } \\
\text { deletion }\end{array}$ & $31 / 34$ & 17 \\
\hline 5 & $\mathrm{NI}$ & $\mathrm{NI}$ & Yes & Total gene deletion & NFI & $\begin{array}{l}\text { MLPA/CGH } \\
\text { array }\end{array}$ & $\begin{array}{l}\text { Genomic } \\
\text { deletion }\end{array}$ & & \\
\hline 24 & $\mathrm{NI}$ & $\mathrm{NI}$ & Yes & EVI20, IVS27, IVS38 & $\begin{array}{l}\text { Intragenic } \\
\mathrm{NFI}\end{array}$ & $\begin{array}{l}\text { MLPA/CGH } \\
\text { array }\end{array}$ & $\begin{array}{l}\text { Genomic } \\
\text { deletion }\end{array}$ & & \\
\hline 26 & $\mathrm{NI}$ & $\mathrm{NI}$ & Yes & NFI gene deletion & NFI & $\begin{array}{l}\text { MLPA/CGH } \\
\text { array }\end{array}$ & $\begin{array}{l}\text { Genomic } \\
\text { deletion }\end{array}$ & & \\
\hline 48 & $\mathrm{NI}$ & $\mathrm{NI}$ & Yes & Determined by MLPA & NFI & MLPA & $\begin{array}{l}\text { Genomic } \\
\text { deletion }\end{array}$ & $6 / 25$ & 18 \\
\hline 86 & $\mathrm{NI}$ & $\mathrm{NI}$ & Yes & Determined by MLPA & NFI & MLPA & $\begin{array}{l}\text { Genomic } \\
\text { deletion }\end{array}$ & & \\
\hline
\end{tabular}


Table SI. Continued

\begin{tabular}{|c|c|c|c|c|c|c|c|c|c|}
\hline $\begin{array}{l}\text { Patient } \\
\text { ID }\end{array}$ & Germline mutation & $\begin{array}{l}\text { Type of } \\
\text { germline } \\
\text { mutation }\end{array}$ & LOH & LOH markers & $\begin{array}{l}\text { Predicted } \\
\text { extent of } \\
\text { LOH }\end{array}$ & $\begin{array}{l}\text { Evidence } \\
\text { for genomic } \\
\text { deletion? } \\
\text { MLPA/CGH } \\
\text { arrayCGH/ } \\
\text { FISH }\end{array}$ & $\begin{array}{l}\text { Probable } \\
\text { mechanism }\end{array}$ & $\begin{array}{l}\text { No. } \\
\text { samples } \\
\text { with } \\
\text { LOH }\end{array}$ & Reference \\
\hline \multicolumn{10}{|l|}{ ACs } \\
\hline T65.I & $\begin{array}{l}\text { Ex24: c. } 4267 \mathrm{~A}>\mathrm{G} \text {; } \\
\text { p.Lys 1 } 423 \mathrm{Glu}\end{array}$ & Missense & Yes & NS & $\begin{array}{l}\text { NFI } 3^{\prime} \\
\text { flanking } \\
\text { region }\end{array}$ & NIA & & $1 / 1$ & 25 \\
\hline 57 & $\mathrm{NI}$ & $\mathrm{NI}$ & Yes & EW206 & $\begin{array}{l}\text { Intragenic } \\
\mathrm{NFI}\end{array}$ & NIA & & $1 / 1$ & 19 \\
\hline 58 & $\mathrm{NI}$ & $\mathrm{NI}$ & Yes & $\begin{array}{l}\text { DI7SI849, DI7SI863, } \\
\text { DI7SI880 }\end{array}$ & $\begin{array}{l}\text { NFI and } 3^{\prime} \\
\text { flanking } \\
\text { region }\end{array}$ & NIA & & $2 / 4$ & 26 \\
\hline 76 & $\mathrm{NI}$ & $\mathrm{NI}$ & Yes & DI7SI863, DI7SI880 & $\begin{array}{l}\text { NFI and } 3^{\prime} \\
\text { flanking } \\
\text { region }\end{array}$ & & & & \\
\hline 182 & $\mathrm{NI}$ & $\mathrm{NI}$ & Yes & IVS27TG24.8 & $\begin{array}{l}\text { Intragenic } \\
\mathrm{NFI}\end{array}$ & NIA & & $11 / 12$ & 27 \\
\hline 185 & $\mathrm{NI}$ & $\mathrm{NI}$ & Yes & $\begin{array}{l}\text { IVS27AC28.4, } \\
\text { IVS38GT53, DI7S804 }\end{array}$ & $\begin{array}{l}\text { NFI region } \\
\text { and some } \\
\text { I7p }\end{array}$ & & & & \\
\hline 187 & $\mathrm{NI}$ & $\mathrm{NI}$ & Yes & $\begin{array}{l}\text { IVS27AC28.4, } \\
\text { IVS38GT53, DI7S796 }\end{array}$ & $\begin{array}{l}\text { NFI region } \\
\text { and some } \\
\text { I7p }\end{array}$ & & & & \\
\hline 309 & $\mathrm{NI}$ & $\mathrm{NI}$ & Yes & IVS38GT53, DI7S796 & $\begin{array}{l}\text { NFI region } \\
\text { and some } \\
17 p\end{array}$ & & & & \\
\hline 330 & $\mathrm{NI}$ & $\mathrm{NI}$ & Yes & $\begin{array}{l}\text { IVS27AC28.4, } \\
\text { IVS38GT53, DI7S520, } \\
\text { DI7S796, DI7S804 }\end{array}$ & $\begin{array}{l}\text { NFI region } \\
\text { and some } \\
\text { I7p }\end{array}$ & & & & \\
\hline
\end{tabular}


Table SI. Continued

$\begin{array}{lllllll}\begin{array}{l}\text { Patient Germline mutation } \\ \text { ID }\end{array} & \begin{array}{l}\text { Type of } \\ \text { germline } \\ \text { mutation }\end{array} & \text { LOH } & \text { LOH markers } & \begin{array}{l}\text { Predicted } \\ \text { extent of } \\ \text { LOH }\end{array} & \begin{array}{l}\text { Evidence } \\ \text { for genomic mechanism } \\ \text { deletion? } \\ \text { MLPA/CGH }\end{array} & \begin{array}{l}\text { No. } \\ \text { samples } \\ \text { with } \\ \text { arrayCGH/ }\end{array} \\ & & & \text { LOH } & \text { Reference } \\ & & & \text { FISH } & \end{array}$

\begin{tabular}{|c|c|c|c|c|c|c|c|c|c|}
\hline 502 & $\mathrm{NI}$ & $\mathrm{NI}$ & Yes & $\begin{array}{l}\text { IVS27AC28.4, DI7S520, } \\
\text { DI7S796 }\end{array}$ & $\begin{array}{l}\text { NFI region } \\
\text { and some } \\
17 \mathrm{p}\end{array}$ & & & & \\
\hline 519 & $\mathrm{NI}$ & $\mathrm{NI}$ & Yes & $\begin{array}{l}\text { IVS27TG28.4, M98509, } \\
\text { IVS27TG24.8, } \\
\text { IVS38GT53 }\end{array}$ & $\begin{array}{l}\text { Intragenic } \\
\mathrm{NFI}\end{array}$ & & & & \\
\hline 297 & $\mathrm{NI}$ & $\mathrm{NI}$ & Yes & $\begin{array}{l}\text { IVS27TG28.4, M98509, } \\
\text { IVS38GT53 }\end{array}$ & $\begin{array}{l}\text { Intragenic } \\
\mathrm{NFI}\end{array}$ & & & & \\
\hline 609 & $\mathrm{NI}$ & $\mathrm{NI}$ & Yes & IVS27TG28.4, M98509 & $\begin{array}{l}\text { Intragenic } \\
\mathrm{NFI}\end{array}$ & & & & \\
\hline 20954 & $\mathrm{NI}$ & $\mathrm{NI}$ & Yes & $\begin{array}{l}\text { IVS27TG24.8, } \\
\text { IVS38GT53 }\end{array}$ & $\begin{array}{l}\text { Intragenic } \\
\mathrm{NFI}\end{array}$ & & & & \\
\hline 20962 & $\mathrm{NI}$ & $\mathrm{NI}$ & Yes & $\begin{array}{l}\text { IVS27AC28.4, M98509, } \\
\text { IVS38GT53 }\end{array}$ & $\begin{array}{l}\text { Intragenic } \\
\text { NFI }\end{array}$ & & & & \\
\hline I & $\mathrm{NI}$ & $\mathrm{NI}$ & Yes & Homozygous & & FISH & Unknown & $3 / 4$ & 28 \\
\hline 9 & $\mathrm{NI}$ & $\mathrm{NI}$ & Yes & Homozygous & & FISH & $\begin{array}{l}\text { Genomic } \\
\text { deletion }\end{array}$ & & \\
\hline 10 & $\mathrm{NI}$ & $\mathrm{NI}$ & Yes & Homozygous & & FISH & $\begin{array}{l}\text { Genomic } \\
\text { deletion }\end{array}$ & & \\
\hline \multicolumn{10}{|c|}{ Gastric carcinoid tumours } \\
\hline I & $\begin{array}{l}\text { Ex37: c.684IC > T; } \\
\text { p.GIn } 228 I X\end{array}$ & Nonsense & Yes & IVS27TG24, DI7S250 & $\begin{array}{l}\text { Intragenic } \\
\mathrm{NFI}\end{array}$ & NIA & & $1 / 1$ & 29 \\
\hline \multicolumn{10}{|l|}{ GISTs } \\
\hline I & $\begin{array}{l}\text { Ex27a: c. } 4537 C>T \text {; } \\
\text { p.Arg15।3X }\end{array}$ & Nonsense & Yes & $\begin{array}{l}\text { DI7S84I, Alu, IVS27GT, } \\
\text { IVS27CAGT, IVS38, } \\
3^{\prime} \mathrm{NFI-I,} \mathrm{3'NFI-2}\end{array}$ & $\begin{array}{l}\mathrm{NFI} \text { and } 3^{\prime} \\
\text { flanking } \\
\text { region }\end{array}$ & MLPA & $\begin{array}{l}\text { Mitotic } \\
\text { recombination }\end{array}$ & $1 / 1$ & 30 \\
\hline
\end{tabular}


Table SI. Continued

\begin{tabular}{|c|c|c|c|c|c|c|c|c|c|}
\hline $\begin{array}{l}\text { Patient } \\
\text { ID }\end{array}$ & Germline mutation & $\begin{array}{l}\text { Type of } \\
\text { germline } \\
\text { mutation }\end{array}$ & LOH & LOH markers & $\begin{array}{l}\text { Predicted } \\
\text { extent of } \\
\text { LOH }\end{array}$ & $\begin{array}{l}\text { Evidence } \\
\text { for genomic } \\
\text { deletion? } \\
\text { MLPA/CGH } \\
\text { arrayCGH/ } \\
\text { FISH }\end{array}$ & $\begin{array}{l}\text { Probable } \\
\text { mechanism }\end{array}$ & $\begin{array}{l}\text { No. } \\
\text { samples } \\
\text { with } \\
\text { LOH }\end{array}$ & Reference \\
\hline NFI-3 & $\begin{array}{l}\text { Ex45: c.7807delG; } \\
\text { p.Aal2603Leufs X3 }\end{array}$ & $\begin{array}{l}\text { I bp } \\
\text { deletion } \\
\text { (FS) }\end{array}$ & Yes & $\begin{array}{l}\text { Alu, IVS27AC33.I, } \\
\text { IVS38GT53.0, } \\
\text { IVS27TG24.8 }\end{array}$ & $\begin{array}{l}\text { Intragenic } \\
\mathrm{NFI}\end{array}$ & Array CGH & $\begin{array}{l}\text { Genomic } \\
\text { deletion }\end{array}$ & $1 / 7$ & 3 \\
\hline \multicolumn{10}{|l|}{ JMML } \\
\hline DIO2 & $\begin{array}{l}\text { Ex4b: c. } 574 C>T \text {; } \\
\text { p.Arg } 192 X\end{array}$ & Nonsense & Yes & $\begin{array}{l}\text { DI7S925, DI7SI800, } \\
\text { DI7SI880, DI7S855, } \\
\text { DI7SI827, DI7S787, } \\
\text { DI7S948, DI7S784 }\end{array}$ & $\begin{array}{l}\text { Majority } \\
\text { of } 17 q\end{array}$ & SNP array & $\begin{array}{l}\text { Mitotic } \\
\text { recombination- } \\
\text { UPD }\end{array}$ & $4 / 5$ & 31 \\
\hline DII5 & $\begin{array}{l}\text { Ex|3: c.2066delT; } \\
\text { p.Val689GlyfsX59 }\end{array}$ & $\begin{array}{l}\text { I bp } \\
\text { deletion } \\
\text { (FS) }\end{array}$ & Yes & $\begin{array}{l}\text { DI7S925, DI7SI800, } \\
\text { DI7SI880, DI7S855, } \\
\text { DI7SI827, DI7S787, } \\
\text { DI7S948, DI7S784 }\end{array}$ & $\begin{array}{l}\text { Majority } \\
\text { of } 17 q\end{array}$ & SNP array & $\begin{array}{l}\text { Mitotic } \\
\text { recombination- } \\
\text { UPD }\end{array}$ & & \\
\hline D003 & $\begin{array}{l}\text { Ex22: c.386I_3862delCT; } \\
\text { p.Cys I I88ValfsX2I }\end{array}$ & $\begin{array}{l}2 \text { bp } \\
\text { deletion } \\
\text { (FS) }\end{array}$ & Yes & $\begin{array}{l}\text { DI7S925, DI7SI800, } \\
\text { DI7SI880, DI7S855, } \\
\text { DI7SI827, DI7S787, } \\
\text { DI7S948, DI7S784 }\end{array}$ & $\begin{array}{l}\text { Majority } \\
\text { of } 17 q\end{array}$ & SNP array & $\begin{array}{l}\text { Mitotic } \\
\text { recombination- } \\
\text { UPD }\end{array}$ & & \\
\hline DI26 & $\begin{array}{l}\text { Ex44: c. } 7699 C>T \text {; } \\
\text { p.Gln2567X }\end{array}$ & Nonsense & Yes & $\begin{array}{l}\text { DI7S925, DI7SI800, } \\
\text { DI7SI880, DI7S855, } \\
\text { DI7SI827, DI7S787, } \\
\text { DI7S948, DI7S784 }\end{array}$ & $\begin{array}{l}\text { Majority } \\
\text { of } 17 q\end{array}$ & SNP array & $\begin{array}{l}\text { Mitotic } \\
\text { recombination- } \\
\text { UPD }\end{array}$ & & \\
\hline I & $\mathrm{NI}$ & $\mathrm{NI}$ & Yes & $\begin{array}{l}\text { DI7SI975, DI7SI294, } \\
\text { UTI72, NFI, } \\
\text { DI7SI800, DI7S250, } \\
\text { DI7S80I, DI7S939, } \\
\text { DI7S836, DI7S1806, } \\
\text { DI7SI822, DI7SI830 }\end{array}$ & $\begin{array}{l}\text { Majority } \\
\text { of } 17 q\end{array}$ & FISH & $\begin{array}{l}\text { Mitotic } \\
\text { recombination- } \\
\text { interstitial } \\
\text { isodisomy } \\
\text { (paternal) }\end{array}$ & $10 / 10$ & 32 \\
\hline
\end{tabular}


Table SI. Continued

\begin{tabular}{|c|c|c|c|c|c|c|c|c|c|}
\hline $\begin{array}{l}\text { Patient } \\
\text { ID }\end{array}$ & Germline mutation & $\begin{array}{l}\text { Type of } \\
\text { germline } \\
\text { mutation }\end{array}$ & LOH & LOH markers & $\begin{array}{l}\text { Predicted } \\
\text { extent of } \\
\text { LOH }\end{array}$ & $\begin{array}{l}\text { Evidence } \\
\text { for genomic } \\
\text { deletion? } \\
\text { MLPA/CGH } \\
\text { arrayCGH/ } \\
\text { FISH }\end{array}$ & $\begin{array}{l}\text { Probable } \\
\text { mechanism }\end{array}$ & $\begin{array}{l}\text { No. } \\
\text { samples } \\
\text { with } \\
\text { LOH }\end{array}$ & Reference \\
\hline 2 & $\mathrm{NI}$ & $\mathrm{NI}$ & Yes & $\begin{array}{l}\text { DI7SI975, DI7SI294, } \\
\text { UTI72, NFI, } \\
\text { DI7SI800, DI7S250, } \\
\text { DI7S80I, DI7S939, } \\
\text { DI7S836, DI7SI806, } \\
\text { DI7SI822, DI7SI830 }\end{array}$ & $\begin{array}{l}\text { Majority } \\
\text { of } 17 q\end{array}$ & FISH & $\begin{array}{l}\text { Mitotic } \\
\text { recombination- } \\
\text { interstitial } \\
\text { isodisomy } \\
\text { (paternal) }\end{array}$ & & \\
\hline 3 & $\mathrm{NI}$ & $\mathrm{NI}$ & Yes & $\begin{array}{l}\text { DI7SI294, UTI72, } \\
\text { NFI, DI7SI800, } \\
\text { DI7S250, DI7S80I, } \\
\text { DI7S939, DI7S836, } \\
\text { DI7SI806, DI7SI822, } \\
\text { DI7SI830 }\end{array}$ & $\begin{array}{l}\text { Majority } \\
\text { of } 17 q\end{array}$ & FISH & $\begin{array}{l}\text { Mitotic } \\
\text { recombination } \\
\text { interstitial } \\
\text { isodisomy } \\
\text { (paternal) }\end{array}$ & & \\
\hline 4 & $\mathrm{NI}$ & $\mathrm{NI}$ & Yes & $\begin{array}{l}\text { DI7SI975, DI7SI294, } \\
\text { UTI72, NFI, } \\
\text { DI7SI800, DI7S250, } \\
\text { DI7S80I, DI7S939, } \\
\text { DI7S836, DI7SI806, } \\
\text { DI7SI822, DI7SI830 }\end{array}$ & $\begin{array}{l}\text { Majority } \\
\text { of } 17 q\end{array}$ & FISH & $\begin{array}{l}\text { Mitotic } \\
\text { recombination- } \\
\text { interstitial } \\
\text { isodisomy } \\
\text { (maternal) }\end{array}$ & & \\
\hline 5 & $\mathrm{NI}$ & $\mathrm{NI}$ & Yes & $\begin{array}{l}\text { DI7SI975, DI7SI294, } \\
\text { UTI72, NFI, } \\
\text { DI7SI800, DI7S250, } \\
\text { DI7S80I, DI7S939, } \\
\text { DI7S836, DI7SI806, } \\
\text { DI7SI822 }\end{array}$ & $\begin{array}{l}\text { Majority of } \\
17 q\end{array}$ & FISH & $\begin{array}{l}\text { Mitotic } \\
\text { recombination- } \\
\text { interstitial } \\
\text { isodisomy } \\
\text { (maternal) }\end{array}$ & & \\
\hline 6 & $\mathrm{NI}$ & $\mathrm{NI}$ & Yes & $\begin{array}{l}\text { DI7SI878, DI7S33, } \\
\text { DI7SI975, DI7SI294, } \\
\text { UTI72, NFI, } \\
\text { DI7SI800, DI7S250, } \\
\text { DI7S80I, DI7S939, } \\
\text { DI7S836, DI7SI806, } \\
\text { DI7SI822, DI7SI830 }\end{array}$ & $\begin{array}{l}\text { Majority of } \\
17 q\end{array}$ & FISH & $\begin{array}{l}\text { Mitotic } \\
\text { recombination- } \\
\text { interstitial } \\
\text { isodisomy } \\
\text { (maternal) }\end{array}$ & & \\
\hline
\end{tabular}


Table SI. Continued

\begin{tabular}{|c|c|c|c|c|c|c|c|c|c|}
\hline $\begin{array}{l}\text { Patient } \\
\text { ID }\end{array}$ & Germline mutation & $\begin{array}{l}\text { Type of } \\
\text { germline } \\
\text { mutation }\end{array}$ & LOH & LOH markers & $\begin{array}{l}\text { Predicted } \\
\text { extent of } \\
\text { LOH }\end{array}$ & $\begin{array}{l}\text { Evidence } \\
\text { for genomic } \\
\text { deletion? } \\
\text { MLPA/CGH } \\
\text { arrayCGH/ } \\
\text { FISH }\end{array}$ & $\begin{array}{l}\text { Probable } \\
\text { mechanism }\end{array}$ & $\begin{array}{l}\text { No. } \\
\text { samples } \\
\text { with } \\
\text { LOH }\end{array}$ & Reference \\
\hline 7 & $\mathrm{NI}$ & $\mathrm{NI}$ & Yes & $\begin{array}{l}\text { DI7SI294, UTI72, } \\
\text { NFI, DI7SI800, } \\
\text { DI7S250, DI7S80I, } \\
\text { DI7S939, DI7S836, } \\
\text { DI7SI806, DI7SI822, } \\
\text { DI7SI830, DI7S928 }\end{array}$ & $\begin{array}{l}\text { Majority of } \\
17 q\end{array}$ & $\mathrm{FISH}$ & $\begin{array}{l}\text { Mitotic } \\
\text { recombination- } \\
\text { interstitial } \\
\text { isodisomy } \\
\text { (maternal) }\end{array}$ & & \\
\hline 8 & $\mathrm{NI}$ & $\mathrm{NI}$ & Yes & $\begin{array}{l}\text { DI7SI975, DI7SI294, } \\
\text { UTI72, NFI, } \\
\text { DI7SI800, DI7S250, } \\
\text { DI7S80I, DI7S939, } \\
\text { DI7S836, DI7SI806, } \\
\text { DI7SI822, DI7SI830, } \\
\text { DI7S928 }\end{array}$ & $\begin{array}{l}\text { Majority of } \\
17 q\end{array}$ & $\mathrm{FISH}$ & $\begin{array}{l}\text { Mitotic } \\
\text { recombination- } \\
\text { interstitial } \\
\text { isodisomy } \\
\text { (paternal) }\end{array}$ & & \\
\hline 9 & $\mathrm{NI}$ & $\mathrm{NI}$ & Yes & NFI, DI7SI800 & $\begin{array}{l}\text { Intragenic } \\
\mathrm{NFI}\end{array}$ & $\mathrm{FISH}$ & $\begin{array}{l}\text { Genomic } \\
\text { deletion }\end{array}$ & & \\
\hline 10 & $\mathrm{NI}$ & $\mathrm{NI}$ & Yes & NFI, DI7SI800 & $\begin{array}{l}\text { Intragenic } \\
\mathrm{NFI}\end{array}$ & $\mathrm{FISH}$ & $\begin{array}{l}\text { Genomic } \\
\text { deletion }\end{array}$ & & \\
\hline D419 & $\mathrm{NI}$ & $\mathrm{NI}$ & Yes & $\begin{array}{l}\text { DI7S925, DI7SI84I, } \\
\text { DI7SI294, DI7SI863, } \\
\text { DI7SI849, DI7SII66, } \\
\text { DI7SI800, DI7SI880, } \\
\text { DI7SI8I8, DI7S855, } \\
\text { DI7SI827, DI7S787, } \\
\text { DI7S948, DI7S785, } \\
\text { DI7S784 }\end{array}$ & $\begin{array}{l}\text { Majority of } \\
17 q\end{array}$ & MLPA & $\begin{array}{l}\text { Mitotic } \\
\text { recombination- } \\
\text { UPD }\end{array}$ & $5 / 10$ & 33 \\
\hline
\end{tabular}


Table SI. Continued

\begin{tabular}{|c|c|c|c|c|c|c|c|c|c|}
\hline $\begin{array}{l}\text { Patient } \\
\text { ID }\end{array}$ & Germline mutation & $\begin{array}{l}\text { Type of } \\
\text { germline } \\
\text { mutation }\end{array}$ & LOH & LOH markers & $\begin{array}{l}\text { Predicted } \\
\text { extent of } \\
\text { LOH }\end{array}$ & $\begin{array}{l}\text { Evidence } \\
\text { for genomic } \\
\text { deletion? } \\
\text { MLPA/CGH } \\
\text { arrayCGH/ } \\
\text { FISH }\end{array}$ & $\begin{array}{l}\text { Probable } \\
\text { mechanism }\end{array}$ & $\begin{array}{l}\text { No. } \\
\text { samples } \\
\text { with } \\
\text { LOH }\end{array}$ & Reference \\
\hline D56 I & $\mathrm{NI}$ & $\mathrm{NI}$ & Yes & $\begin{array}{l}\text { DI7SI294, DI7SI863, } \\
\text { DI7SI849, DI7SII66, } \\
\text { DI7SI800, DI7SI880, } \\
\text { DI7SI8I8, DI7S855, } \\
\text { DI7SI827, DI7S787, } \\
\text { DI7S948, DI7S785, } \\
\text { DI7S784 }\end{array}$ & $\begin{array}{l}\text { Majority of } \\
17 q\end{array}$ & MLPA & $\begin{array}{l}\text { Mitotic } \\
\text { recombination- } \\
\text { UPD }\end{array}$ & & \\
\hline D378 & $\mathrm{NI}$ & $\mathrm{NI}$ & Yes & $\begin{array}{l}\text { DI7SI294, DI7SI863, } \\
\text { DI7SI849, DI7SII66, } \\
\text { DI7SI800, DI7SI880, } \\
\text { DI7SI8I8, DI7S855, } \\
\text { DI7S785 }\end{array}$ & $\begin{array}{l}\text { Majority of } \\
17 q\end{array}$ & Array CGH & $\begin{array}{l}\text { Mitotic } \\
\text { recombination- } \\
\text { UPD }\end{array}$ & & \\
\hline D34 I & $\mathrm{NI}$ & $\mathrm{NI}$ & Yes & $\begin{array}{l}\text { DI7SI849, DI7SII66, } \\
\text { DI7SI800, DI7SI880 }\end{array}$ & $\begin{array}{l}\mathrm{NFI} \text { and } \\
\text { flanking } \\
\text { regions }\end{array}$ & Array CGH & $\begin{array}{l}\text { Genomic } \\
\text { deletion }\end{array}$ & & \\
\hline D566 & $\mathrm{NI}$ & $\mathrm{NI}$ & Yes & $\begin{array}{l}\text { DI7SI849, DI7SII66, } \\
\text { DI7SI800, DI7S784 }\end{array}$ & $\begin{array}{l}\mathrm{NFI} \text { and } \\
\text { flanking } \\
\text { regions }\end{array}$ & Array CGH & $\begin{array}{l}\text { Genomic } \\
\text { deletion }\end{array}$ & & \\
\hline \multicolumn{10}{|l|}{ PCs } \\
\hline I & $\mathrm{NI}$ & $\mathrm{NI}$ & Yes & $\begin{array}{l}\text { DI7S34, DI7SI37, } \\
\text { CRYBI, NFI, DI7S4 }\end{array}$ & $\begin{array}{l}\text { Whole } \\
\text { chromosome }\end{array}$ & NIA & & $7 / 7$ & 22 \\
\hline 2 & $\mathrm{NI}$ & $\mathrm{NI}$ & Yes & $\begin{array}{l}\text { CRYBI, DI7S33, NFI, } \\
\text { DI7S55, DI7S4 }\end{array}$ & NFI & & & & \\
\hline 3 & $\mathrm{NI}$ & $\mathrm{NI}$ & Yes & $\begin{array}{l}\text { DI7S5, DI7SI34, } \\
\text { DI7S58, DI7S33 }\end{array}$ & $\begin{array}{l}\text { Whole } \\
\text { chromosome }\end{array}$ & & & & \\
\hline 4 & $\mathrm{NI}$ & $\mathrm{NI}$ & Yes & DI7S33, NFI & $\begin{array}{l}\text { Intragenic } \\
\mathrm{NFI}\end{array}$ & & & & \\
\hline 5 & $\mathrm{NI}$ & $\mathrm{NI}$ & Yes & DI7S7I, NFI, DI7S226 & $\begin{array}{l}\text { Whole } \\
\text { chromosome }\end{array}$ & & & & \\
\hline
\end{tabular}


Table SI. Continued

\begin{tabular}{|c|c|c|c|c|c|c|c|c|c|}
\hline $\begin{array}{l}\text { Patient } \\
\text { ID }\end{array}$ & Germline mutation & $\begin{array}{l}\text { Type of } \\
\text { germline } \\
\text { mutation }\end{array}$ & LOH & LOH markers & $\begin{array}{l}\text { Predicted } \\
\text { extent of } \\
\text { LOH }\end{array}$ & $\begin{array}{l}\text { Evidence } \\
\text { for genomic } \\
\text { deletion? } \\
\text { MLPA/CGH } \\
\text { arrayCGH/ } \\
\text { FISH }\end{array}$ & $\begin{array}{l}\text { Probable } \\
\text { mechanism }\end{array}$ & $\begin{array}{l}\text { No. } \\
\text { samples } \\
\text { with } \\
\text { LOH }\end{array}$ & Reference \\
\hline
\end{tabular}

\begin{tabular}{|c|c|c|c|c|c|c|c|c|c|}
\hline $6 \mathrm{~L}$ & $\mathrm{NI}$ & $\mathrm{NI}$ & Yes & $\begin{array}{l}\text { DI7S5, NFI, DI7SI45, } \\
\text { DI7S226 }\end{array}$ & $\begin{array}{l}\text { Whole } \\
\text { chromosome }\end{array}$ & & & & \\
\hline 6R & $\mathrm{NI}$ & $\mathrm{NI}$ & Yes & $\begin{array}{l}\text { DI7S5, NFI, DI7SI45, } \\
\text { DI7S226 }\end{array}$ & $\begin{array}{l}\text { Whole } \\
\text { chromosome }\end{array}$ & & & & \\
\hline I & $\mathrm{NI}$ & $\mathrm{NI}$ & Yes & $\begin{array}{l}\text { TP53-BAM, TP53 Accll, } \\
\text { NFI-AE25 (BgllI) SNP, } \\
\text { THH59-Taql, } \\
\text { THH59-Pvull }\end{array}$ & $\begin{array}{l}\text { Majority of } \\
17\end{array}$ & NIA & & $2 / 7$ & 34 \\
\hline I & $\mathrm{NI}$ & $\mathrm{NI}$ & Yes & $\begin{array}{l}\text { NFI-AE25 (BgllI) SNP, } \\
\text { THH59-Taql, THH59- } \\
\text { Pvull-adrenal corticoid } \\
\text { tumour }\end{array}$ & $\begin{array}{l}\text { NFI and 3' } \\
\text { flanking } \\
\text { region }\end{array}$ & & & & \\
\hline NS & $\mathrm{NI}$ & $\mathrm{NI}$ & Yes & & & & & $|4 / 2|$ & 35 \\
\hline \multicolumn{10}{|c|}{ Glomus tumours } \\
\hline NFI-G2 & $\begin{array}{l}\text { Ex42: c.7395_7404dell 0; } \\
\text { p.Thr2466SerfsX33 }\end{array}$ & $\begin{array}{l}10 \text { bp } \\
\text { deletion } \\
\text { (FS) }\end{array}$ & Yes & Introns $27-38$ & $\begin{array}{l}\text { Intragenic } \\
\mathrm{NFI}\end{array}$ & Array CGH & $\begin{array}{l}\text { Mitotic } \\
\text { recombination }\end{array}$ & 1/7 & 36 \\
\hline
\end{tabular}

CGH, comparative genomic hybridisation; array CGH, high resolution CGH; FS, frame shift; NI, not informative; WG, whole gene; NA, not available; UPD, uniparental disomy; MLPA, multiplex ligation-dependent probe amplification; FISH, fluorescent in situ hybridisation. 
Table S2. Summary of germline and somatic point mutations in NFI-associated tumours

\begin{tabular}{|c|c|c|c|c|c|}
\hline $\begin{array}{l}\text { Patient } \\
\text { ID }\end{array}$ & $\begin{array}{l}\text { Germline point } \\
\text { mutation }\end{array}$ & $\begin{array}{l}\text { Type of } \\
\text { germline } \\
\text { mutation }\end{array}$ & $\begin{array}{l}\text { Somatic point } \\
\text { mutation }\end{array}$ & $\begin{array}{l}\text { Effect of } \\
\text { somatic } \\
\text { mutation }\end{array}$ & Source \\
\hline \multicolumn{6}{|c|}{ Dermal neurofibromas } \\
\hline T196.3 & Ex 2 and 3 deleted & $\begin{array}{l}2 \text { exon } \\
\text { deletion }\end{array}$ & $\begin{array}{l}\text { Ex4c: c.648dup73 p.Leu2 I6 } \\
\text { (through splice site) }\end{array}$ & $\begin{array}{l}73 \text { bp } \\
\text { duplication } \\
\text { (FS) }\end{array}$ & I \\
\hline T196.12 & & & Ex4c: c.655-IG > A & Splice site & \\
\hline T196.15 & & & $\begin{array}{l}\text { Ex6: c.750delT } \\
\text { p.Phe250Leufs } \times 30\end{array}$ & $\begin{array}{l}\text { I bp deletion } \\
\text { (FS) }\end{array}$ & \\
\hline T196.16 & & & $\begin{array}{l}\text { ExI6: c. } 2534 \_2557 \text { del24 } \\
\text { p.Cys } 845 X\end{array}$ & $\begin{array}{l}24 \text { bp deletion } \\
\text { (In-frame) }\end{array}$ & \\
\hline T196.7 & & & $\begin{array}{l}\text { ExI6: c.2844delA } \\
\text { p.Gly949AspfX3 }\end{array}$ & $\begin{array}{l}\text { I bp deletion } \\
\text { (FS) }\end{array}$ & \\
\hline TI96.4 & & & $\begin{array}{l}\text { ExI8: c.3047_c3048delGT } \\
\text { p.Cys I0I6SerfsX4 }\end{array}$ & $\begin{array}{l}2 \text { bp deletion } \\
\text { (FS) }\end{array}$ & \\
\hline T196.5 & & & $\begin{array}{l}\text { Ex27a: c. } 4537 C>T \\
\text { p.Argl5I3X } \underline{R}\end{array}$ & Nonsense & \\
\hline T196.13 & & & $\begin{array}{l}\text { Ex27b: c. } 4743 \text { delG } \\
\text { p.Asp I582llefs X2I }\end{array}$ & $\begin{array}{l}\text { I bp deletion } \\
\text { (FS) }\end{array}$ & \\
\hline TI96.I & & & $\begin{array}{l}\text { Ex44: c.772I_7722delAA } \\
\text { p.Lys257Ser4fs X4 }\end{array}$ & $\begin{array}{l}2 \text { bp deletion } \\
\text { (FS) }\end{array}$ & \\
\hline T543.I & $\begin{array}{l}\text { Ex4a: c. } 373 \text { delGinsATGTGT } \\
\text { p.Arg } 125 \mathrm{Hisfs} \times 22\end{array}$ & Indel (FS) & $\begin{array}{l}\text { Ex2I: c.3568del } 80 \\
\text { p.GlyI I } 90 \text { HisfsX3 }\end{array}$ & $\begin{array}{l}80 \text { bp deletion } \\
\text { (FS) }\end{array}$ & $\begin{array}{l}\text { Unpublished data, } \\
\text { Cardiff }\end{array}$ \\
\hline T543.3 & & & $\begin{array}{l}\text { Ex26: c.4388C > T } \\
\text { p.Ser 1463Phe }\end{array}$ & Missense & \\
\hline TI28.10 & Ex6: c.784C > T p.Arg262Cys & Missense & $\begin{array}{l}\text { Ex4b: c. } 574 C>T \text { p.Arg } 192 X \\
\underline{R}\end{array}$ & Nonsense & $\begin{array}{l}\text { Unpublished data, } \\
\text { Cardiff }\end{array}$ \\
\hline TI28.I & & & $\begin{array}{l}\text { Ex8: c. I I 70delC } \\
\text { p.Asp390LysfsX6 }\end{array}$ & $\begin{array}{l}\text { I bp deletion } \\
\text { (FS) }\end{array}$ & \\
\hline
\end{tabular}


Table S2. Continued

\begin{tabular}{|c|c|c|c|c|c|}
\hline $\begin{array}{l}\text { Patient } \\
\text { ID }\end{array}$ & $\begin{array}{l}\text { Germline point } \\
\text { mutation }\end{array}$ & $\begin{array}{l}\text { Type of } \\
\text { germline } \\
\text { mutation }\end{array}$ & $\begin{array}{l}\text { Somatic point } \\
\text { mutation }\end{array}$ & $\begin{array}{l}\text { Effect of } \\
\text { somatic } \\
\text { mutation }\end{array}$ & Source \\
\hline TI28.17 & & & $\begin{array}{l}\text { ExI0c: c.I556A >C } \\
\text { p.Gln5I9Pro } \underline{R}\end{array}$ & Missense & \\
\hline TI28.8 & & & $\begin{array}{l}\text { Ex32: c.6055_6056delTC } \\
\text { p.Ser2019TrpfsXI8 }\end{array}$ & $\begin{array}{l}2 \text { bp deletion } \\
\text { (FS) }\end{array}$ & \\
\hline NF29a-4 & Ex6: c.80IG > A p.Trp267X & Nonsense & $\begin{array}{l}\text { ExI0a: c. }|38| C>T \\
\text { p.Arg46IX }\end{array}$ & Nonsense & 37 \\
\hline NFI7-8 & & & $\begin{array}{l}\text { ExI0c: c.I528-I4_I546del33 } \\
\text { p.Asp5 I0fs (through splice } \\
\text { site) }\end{array}$ & $\begin{array}{l}32 \text { bp deletion } \\
{[\mathrm{FS}]}\end{array}$ & \\
\hline NFI7-I & & & ExI0c: c. $|64|+\mid G>A$ & Splice site & \\
\hline NFI7-9 & & & $\begin{array}{l}\text { ExI8: c. } 3049 C>T \\
\text { p.Glul017X }\end{array}$ & Nonsense & \\
\hline NF29a-7 & & & $\begin{array}{l}\text { Ex|9b: c.3303_33I4+7dell9 } \\
\text { p.GluII0I (through splice site) }\end{array}$ & $\begin{array}{l}19 \text { bp deletion } \\
{[\mathrm{FS}]}\end{array}$ & \\
\hline NFI7-I5 & & & $\begin{array}{l}\text { Ex23.I: c.3916C>T } \\
\text { p.Argl306X } \underline{R}\end{array}$ & Nonsense & \\
\hline NF29a-9 & & & $\begin{array}{l}\text { Ex27b: c.4756insT } \\
\text { p.Tyr I586LeufsXI4 }\end{array}$ & $\begin{array}{l}\text { I bp insertion } \\
\text { (FS) }\end{array}$ & \\
\hline NFI7-I8 & & & Ex28: c.5205 + IG > A & Splice site & \\
\hline NFI $7-23$ & & & $\begin{array}{l}\text { Ex3।: c.5772_5775delTTTG } \\
\text { p.Cys 1924TrpfsX4 }\end{array}$ & $\begin{array}{l}4 \text { bp deletion } \\
\text { (FS) }\end{array}$ & \\
\hline NF29a-5 & & & $\begin{array}{l}\text { Ex40: c.7237_7253dell } 7 \\
\text { p.Gln } 24 \mid 3 \text { fs } X 2\end{array}$ & $\begin{array}{l}\text { I } 7 \text { bp deletion } \\
\text { (FS) }\end{array}$ & \\
\hline L-002 F & Ex9: c. I246C > T p.Arg4I6X & Nonsense & $\begin{array}{l}\text { Ex3: c.246_247delTC } \\
\text { p.Glu83Serfs X15 }\end{array}$ & $\begin{array}{l}2 \text { bp deletion } \\
\text { (FS) }\end{array}$ & 3 \\
\hline L-002 A & & & Ex5: c.655-IG > T & Splice site & \\
\hline L-002 D & & & Ex8: c.II05C > T p.Gln369X & Nonsense & \\
\hline
\end{tabular}


Table S2. Continued

\begin{tabular}{|c|c|c|c|c|c|}
\hline $\begin{array}{l}\text { Patient } \\
\text { ID }\end{array}$ & $\begin{array}{l}\text { Germline point } \\
\text { mutation }\end{array}$ & $\begin{array}{l}\text { Type of } \\
\text { germline } \\
\text { mutation }\end{array}$ & $\begin{array}{l}\text { Somatic point } \\
\text { mutation }\end{array}$ & $\begin{array}{l}\text { Effect of } \\
\text { somatic } \\
\text { mutation }\end{array}$ & Source \\
\hline L-002 E & & & $\begin{array}{l}\text { Ex8: c.I I53delC } \\
\text { p.Arg385AlafsX2 }\end{array}$ & $\begin{array}{l}\text { I bp deletion } \\
\text { (FS) }\end{array}$ & \\
\hline L-002 B & & & $\begin{array}{l}\text { Ex22: c.3757_3764del8 } \\
\text { p.Leul253ThrfsX8 }\end{array}$ & $\begin{array}{l}8 \text { bp deletion } \\
\text { (FS) }\end{array}$ & \\
\hline NF282-I & Ex9: c. $1260+I G>A$ & Splice site & $\begin{array}{l}\text { Ex23.2: c. } 402 I C>T \\
\text { p.Gln } 134 I X\end{array}$ & Nonsense & 8 \\
\hline NF282-2 & & & $\begin{array}{l}\text { Ex23.2: c.4084C }>T \\
\text { p.Argl } 362 X\end{array}$ & Nonsense & \\
\hline T473.6 & $\begin{array}{l}\text { ExI0b: c. I413_|4|4delAG } \\
\text { p.Lys47|AsnfsXI }\end{array}$ & $\begin{array}{l}2 \text { bp deletion } \\
\text { (FS) }\end{array}$ & $\begin{array}{l}\text { Ex7: c.890delA } \\
\text { p.Leu297Serfs X20 }\end{array}$ & $\begin{array}{l}\text { I bp deletion } \\
\text { (FS) }\end{array}$ & 4 \\
\hline T473.12 & & & Ex|2b: c.1884insA p.Tyr628X & $\begin{array}{l}\text { I bp insertion } \\
\text { (FS) }\end{array}$ & \\
\hline T473.1I & & & $\begin{array}{l}\text { Ex|6: c. } 245 \text { IinsG } \\
\text { p.Ser8|8Valfs } X 12\end{array}$ & $\begin{array}{l}\text { I bp insertion } \\
\text { (FS) }\end{array}$ & \\
\hline T473.18 & & & $\begin{array}{l}\text { Ex22: c.3807insC } \\
\text { p.Ser I270Leufs XI3 }\end{array}$ & $\begin{array}{l}\text { I bp insertion } \\
\text { (FS) }\end{array}$ & \\
\hline T473.20 & & & $\begin{array}{l}\text { Ex23.2: c.4087delA } \\
\text { p.Ser|363Valfs X22 }\end{array}$ & $\begin{array}{l}\text { I bp deletion } \\
\text { (FS) }\end{array}$ & \\
\hline T473.13 & & & $\begin{array}{l}\text { Ex3I: c.5888A >C } \\
\text { p.Asn 1963Thr }\end{array}$ & Missense & \\
\hline T473.33 & & & $\begin{array}{l}\text { Ex34: c.6478A > G } \\
\text { p.Ser2160Gly }\end{array}$ & Missense & \\
\hline T473.36 & & & $\begin{array}{l}\text { Ex38: c.6859delG } \\
\text { p.Asp2287Thrfs X18 }\end{array}$ & $\begin{array}{l}\text { I bp deletion } \\
\text { (FS) }\end{array}$ & \\
\hline T473.17 & & & $\begin{array}{l}\text { Ex40: c.7I28delG } \\
\text { p.Tyr2377Thrfs X23 }\end{array}$ & $\begin{array}{l}\text { I bp deletion } \\
\text { (FS) }\end{array}$ & \\
\hline T82.3 & $\begin{array}{l}\text { Ex।2a: c.1754_I757delTAAC } \\
\text { p.Thr585Valfs X18 }\end{array}$ & $\begin{array}{l}4 \text { bp deletion } \\
\text { (FS) }\end{array}$ & $\begin{array}{l}\text { Ex|6: c.2445delG } \\
\text { p.Arg8I5Serfs X5 }\end{array}$ & $\begin{array}{l}\text { I bp deletion } \\
\text { (FS) }\end{array}$ & $\begin{array}{l}\text { Unpublished data, } \\
\text { Cardiff }\end{array}$ \\
\hline
\end{tabular}


Table S2. Continued

Patient Germline point

ID mutation

\begin{tabular}{|c|c|c|c|c|c|}
\hline T82.5 & & & $\begin{array}{l}\text { Ex35: c.662I_6625delGTGGA } \\
\text { p.GIn2207HisfsXII }\end{array}$ & $\begin{array}{l}5 \text { bp deletion } \\
\text { (FS) }\end{array}$ & \\
\hline T77.3 & $\begin{array}{l}\text { ExI2a: c. } 1783 \mathrm{G}>\mathrm{A} \\
\text { p.Glu595Lys }\end{array}$ & Missense & $\begin{array}{l}\text { ExI6: c. } 2446 C>T \\
\text { p.Arg8I6X } \underline{R}\end{array}$ & Nonsense & $\begin{array}{l}\text { Unpublished data, } \\
\text { Cardiff }\end{array}$ \\
\hline T77.I & & & $\begin{array}{l}\text { Ex29: c.5242C }>T \\
\text { p.Arg I } 748 \times \underline{R}\end{array}$ & Nonsense & \\
\hline T77.4 & & & $\begin{array}{l}\text { Ex3І: c. } 5839 \mathrm{C}>\mathrm{T} \\
\text { p.Argl } 947 \mathrm{X}\end{array}$ & Nonsense & \\
\hline TI4I.4 & $\begin{array}{l}\text { Ex|3: c. } 2233 \text { delA } \\
\text { p.Ser745Alafs X2 }\end{array}$ & $\begin{array}{l}\text { I bp deletion } \\
\text { (FS) }\end{array}$ & $\begin{array}{l}\text { ExI2b: c. } 1885 G>A \\
\text { p.Gly629Arg }\end{array}$ & Missense & $\begin{array}{l}\text { Unpublished data, } \\
\text { Cardiff }\end{array}$ \\
\hline TI4I.13 & & & $\begin{array}{l}\text { Ex30: c. } 573 \text { I delT } \\
\text { p.Ser I9I ILeufsX9 } \underline{R}\end{array}$ & $\begin{array}{l}2 \text { bp deletion } \\
\text { (FS) }\end{array}$ & \\
\hline TI33 & $\begin{array}{l}\text { ExI6: c. } 2446 C>T \\
\text { p.Arg8I } 6 X\end{array}$ & Nonsense & $\begin{array}{l}\text { Ex3I: c.5897dupAC } \\
\text { p.GluI } 966 \text { Hisfs } \times 25\end{array}$ & $\begin{array}{l}2 \text { bp } \\
\text { duplication } \\
\text { (FS) }\end{array}$ & $\begin{array}{l}\text { Unpublished data, } \\
\text { Cardiff }\end{array}$ \\
\hline TI37 & & & $\begin{array}{l}\text { Ex3I: c.5898dupAC } \\
\text { p.Glul } 966 \text { Hisfs X25 }\end{array}$ & $\begin{array}{l}2 \text { bp } \\
\text { duplication } \\
\text { (FS) }\end{array}$ & \\
\hline T437 & $\begin{array}{l}\text { ExI7: c. } 2875 C>T \\
\text { p.Gln } 959 X\end{array}$ & Nonsense & Ex2: c.67A > T p.lle23Leu & Missense & 4 \\
\hline T44। & & & Ex4b: c.586G > T p.Glu196X & Nonsense & \\
\hline T459 & & & ExI0c: c. $1641+2 T>G$ & Splice site & \\
\hline T433 & & & $\begin{array}{l}\text { ExI0c: c. } 1660 \mathrm{C}>\mathrm{G} \\
\text { p.Gln554Glu }\end{array}$ & Missense & \\
\hline T469 & & & $\begin{array}{l}\text { ExI2a: c. I724delCACA } \\
\text { p.Ser575X }\end{array}$ & $\begin{array}{l}4 \text { bp deletion } \\
\text { (FS) }\end{array}$ & \\
\hline T468 & & & ExI3: c.204IC > T p.Arg68IX & Nonsense & \\
\hline T472 & & & Ex|3: c.2088G > A p.Trp696X & Nonsense & \\
\hline
\end{tabular}

Type of germline

Somatic point mutation

Effect of

somatic

somatic

Source 
Table S2. Continued

\begin{tabular}{|c|c|c|c|c|c|}
\hline $\begin{array}{l}\text { Patient } \\
\text { ID }\end{array}$ & $\begin{array}{l}\text { Germline point } \\
\text { mutation }\end{array}$ & $\begin{array}{l}\text { Type of } \\
\text { germline } \\
\text { mutation }\end{array}$ & $\begin{array}{l}\text { Somatic point } \\
\text { mutation }\end{array}$ & $\begin{array}{l}\text { Effect of } \\
\text { somatic } \\
\text { mutation }\end{array}$ & Source \\
\hline T463 & & & Ex|6: c.24|0-3T $>G$ & Splice site & \\
\hline T45I & & & $\begin{array}{l}\text { Ex20: c.3449C }>\text { T } \\
\text { p.Serl I50Leu }\end{array}$ & Missense & \\
\hline T456 & & & Ex22: c.3709-2A > G & Splice site & \\
\hline T450 & & & $\begin{array}{l}\text { Ex23.2: c. } 4084 \mathrm{C}>\mathrm{T} \\
\text { p.Argl } 362 \times \underline{R}\end{array}$ & Nonsense & \\
\hline T442 & & & $\begin{array}{l}\text { Ex27b: c.4687_469/ del5 } \\
\text { p.Phe I563GlyfsX36 }\end{array}$ & $\begin{array}{l}5 \text { bp deletion } \\
\text { (FS) }\end{array}$ & \\
\hline T443 & & & $\begin{array}{l}\text { Ex27b: c.4693insG } \\
\text { p.Ala I565GlyfsX35 }\end{array}$ & $\begin{array}{l}\text { I bp insertion } \\
\text { (FS) }\end{array}$ & \\
\hline T467 & & & $\begin{array}{l}\text { Ex29: c.5380C }>T \\
\text { p.Gln I794X }\end{array}$ & Nonsense & \\
\hline T457 & & & $\begin{array}{l}\text { Ex34: c.6448A > T } \\
\text { p.Lys2 I I0X }\end{array}$ & Nonsense & \\
\hline T47I & & & $\begin{array}{l}\text { Ex38: c.6895delG } \\
\text { p.Val2299Trpfs X8 }\end{array}$ & $\begin{array}{l}\text { I bp deletion } \\
\text { (FS) }\end{array}$ & \\
\hline T434 & & & $\begin{array}{l}\text { Ex44: c.7699C }>T \\
\text { p.Gln } 2567 X\end{array}$ & Nonsense & \\
\hline T435 & & & $\begin{array}{l}\text { Ex44: c.7702C }>T \\
\text { p.Gln } 2568 X\end{array}$ & Nonsense & \\
\hline T460 & & & $\begin{array}{l}\text { Ex46: c.7924delT } \\
\text { p.Ser2642LeufsXI6 }\end{array}$ & $\begin{array}{l}\text { I bp deletion } \\
\text { (FS) }\end{array}$ & \\
\hline CSG6N & $\begin{array}{l}\text { Ex21: c.3525_3526delAA } \\
\text { p.Argl I76SerfsX18 }\end{array}$ & $\begin{array}{l}2 \text { bp deletion } \\
\text { (FS) }\end{array}$ & $\begin{array}{l}\text { Ex4c: c.587-8del6 Splicing } \\
\text { effect? }\end{array}$ & $\begin{array}{l}\text { Intronic } \\
\text { deletion }\end{array}$ & 6,7 \\
\hline CSGI3N & & & Ex9: c. $1260+$ IG $>A$ & Splice site & \\
\hline CSG48N & & & $\begin{array}{l}\text { Exl0c: c. } 1604 \mathrm{~A}>\mathrm{G} \\
\text { p.Gln535Arg }\end{array}$ & Missense & \\
\hline
\end{tabular}


Table S2. Continued

\begin{tabular}{|c|c|c|c|c|c|}
\hline $\begin{array}{l}\text { Patient } \\
\text { ID }\end{array}$ & $\begin{array}{l}\text { Germline point } \\
\text { mutation }\end{array}$ & $\begin{array}{l}\text { Type of } \\
\text { germline } \\
\text { mutation }\end{array}$ & $\begin{array}{l}\text { Somatic point } \\
\text { mutation }\end{array}$ & $\begin{array}{l}\text { Effect of } \\
\text { somatic } \\
\text { mutation }\end{array}$ & Source \\
\hline CSG29N & & & Ex|4: c.2266C > T p.Gln756X & Nonsense & \\
\hline CSG33N & & & $\begin{array}{l}\text { ExI6: c. } 2816 \text { delA } \\
\text { p.Asn939llefsXI2 }\end{array}$ & $\begin{array}{l}\text { I bp deletion } \\
\text { (FS) }\end{array}$ & \\
\hline CSGI9N & & & $\begin{array}{l}\text { ExI7: c. } 2928 \text { dell } 3 \\
\text { p.Glu977AsnfsX3 }\end{array}$ & $\begin{array}{l}\text { I } 3 \text { bp deletion } \\
\text { (FS) }\end{array}$ & \\
\hline CSG26N & & & Ex26: c. $4514+I G>C$ & Splice site & \\
\hline CSG44N & & & $\begin{array}{l}\text { Ex3 I: c.5774delT } \\
\text { p.Leul 925TrpfsX4 }\end{array}$ & $\begin{array}{l}\text { I bp deletion } \\
\text { (FS) }\end{array}$ & \\
\hline CSG8N & & & $\begin{array}{l}\text { Ex33: c.6292_6322del3I } \\
\text { p.Arg2098Phefs X2I }\end{array}$ & $\begin{array}{l}31 \text { bp deletion } \\
\text { (FS) }\end{array}$ & \\
\hline CSG30N & & & Ex45: c.7908-2A $>T$ & Splice site & \\
\hline $\begin{array}{l}\text { NF482- } \\
\text { UHG B }\end{array}$ & $\begin{array}{l}\text { Ex2I: c.3525_3526delAA } \\
\text { p.ArgI I } 76 \text { SerfsXI8 }\end{array}$ & $\begin{array}{l}2 \text { bp deletion } \\
\text { (FS) }\end{array}$ & $\begin{array}{l}\text { Ex4a: c.359_375dell7 } \\
\text { p.Phel20X }\end{array}$ & $\begin{array}{l}\text { I7 bp deletion } \\
\text { (FS) }\end{array}$ & 3 \\
\hline $\begin{array}{l}\text { NF482- } \\
\text { UHG C }\end{array}$ & & & $\begin{array}{l}\text { Ex4c: c.603_62I dell9 } \\
\text { p.Phe20IfsX4 }\end{array}$ & $\begin{array}{l}19 \text { bp deletion } \\
\text { (FS) }\end{array}$ & \\
\hline $\begin{array}{l}\text { NF482- } \\
\text { UHG A }\end{array}$ & & & Ex8: c. $1185+1 G>A$ & Splice site & \\
\hline $\begin{array}{l}\text { NF482- } \\
\text { UHG D }\end{array}$ & & & $\begin{array}{l}\text { Ex|4: c.2252-30_2252- } \\
\text { 6del??insT }\end{array}$ & Indel (FS?) & \\
\hline T191.5 & $\begin{array}{l}\text { Ex22: c. } 372 \mid \mathrm{C}>\mathrm{T} \\
\text { p.Argl } 24 \mid X\end{array}$ & Nonsense & $\begin{array}{l}\text { Ex4b: c.505_524del20 } \\
\text { p.Glul69X }\end{array}$ & $\begin{array}{l}20 \text { bp deletion } \\
\text { (FS) }\end{array}$ & $\begin{array}{l}\text { Unpublished data, } \\
\text { Cardiff }\end{array}$ \\
\hline T191.9 & & & $\begin{array}{l}\text { ExI0b: c. } 14 \mid 7 \text { delA } \\
\text { p.Thr473GInfs } X 24\end{array}$ & $\begin{array}{l}\text { I bp deletion } \\
\text { (FS) }\end{array}$ & \\
\hline TI9I.I & & & ExI8: c.299I + IG >A & Splice site & \\
\hline T191.2 & & & $\begin{array}{l}\text { Ex22: c. } 372 \mid C>T \\
\text { p.ArgI } 24 \mid X \underline{R}\end{array}$ & Nonsense & \\
\hline
\end{tabular}


Table S2. Continued

\begin{tabular}{|c|c|c|c|c|c|}
\hline $\begin{array}{l}\text { Patient } \\
\text { ID }\end{array}$ & $\begin{array}{l}\text { Germline point } \\
\text { mutation }\end{array}$ & $\begin{array}{l}\text { Type of } \\
\text { germline } \\
\text { mutation }\end{array}$ & $\begin{array}{l}\text { Somatic point } \\
\text { mutation }\end{array}$ & $\begin{array}{l}\text { Effect of } \\
\text { somatic } \\
\text { mutation }\end{array}$ & Source \\
\hline TI75.I & $\begin{array}{l}\text { Ex23.2: c. } 4084 C>T \\
\text { p.ArgI } 362 X\end{array}$ & Nonsense & $\begin{array}{l}\text { ExI2a: c. 1738insT } \\
\text { p. Tyr580Leufs X7 R }\end{array}$ & $\begin{array}{l}\text { I bp insertion } \\
\text { (FS) }\end{array}$ & $\begin{array}{l}\text { Unpublished data, } \\
\text { Cardiff }\end{array}$ \\
\hline TI75.2 & & & $\begin{array}{l}\text { Ex3I: c. } 5817 C>A \\
\text { p.Cys1939X R }\end{array}$ & Nonsense & \\
\hline T209. Iii & $\begin{array}{l}\text { Ex28: c. } 4950 C>A \\
\text { p.Tyr } 1650 X\end{array}$ & Nonsense & Ex7: c. $1062+$ IG $>$ A $\underline{R}$ & Splice site & $\begin{array}{l}\text { Unpublished data, } \\
\text { Cardiff }\end{array}$ \\
\hline T209.7 & & & $\begin{array}{l}\text { Ex|0a: c. I318C > T } \\
\text { p.Arg440X } \underline{R}\end{array}$ & Nonsense & \\
\hline T209.8 & & & $\begin{array}{l}\text { ExI5: c. } 2326 \mathrm{G}>A \\
\text { p. Ala776Thr } \underline{\mathrm{R}}\end{array}$ & $\begin{array}{l}\text { Missense? / } \\
\text { splicing? }\end{array}$ & \\
\hline T209.5 & & & $\begin{array}{l}\text { Ex25: c.4345delA } \\
\text { p.Ser I 449Alafs X12 }\end{array}$ & $\begin{array}{l}\text { I bp deletion } \\
\text { (FS) }\end{array}$ & \\
\hline T209.6 & & & $\begin{array}{l}\text { Ex37: c.6790_6806del } 17 \\
\text { p.Tyr2264AspfsX8 }\end{array}$ & $\begin{array}{l}17 \text { bp deletion } \\
\text { (FS) }\end{array}$ & \\
\hline T506.5 & Ex36: c.6756 + 2T > G & Splice site & $\begin{array}{l}\text { Ex4b: c.480delG } \\
\text { p.ArgI60Serfs X5 }\end{array}$ & $\begin{array}{l}\text { I bp deletion } \\
\text { (FS) }\end{array}$ & 4 \\
\hline T506.2 & & & $\begin{array}{l}\text { Ex6: c.73I_732delAA } \\
\text { p.Glu244Valfs X5 }\end{array}$ & $\begin{array}{l}2 \text { bp deletion } \\
\text { (FS) }\end{array}$ & \\
\hline T506.4 & & & $\begin{array}{l}\text { ExI7: c.2987insAC } \\
\text { p.Val996Aspfs XI7 }\end{array}$ & $\begin{array}{l}2 \text { bp insertion } \\
\text { (FS) }\end{array}$ & \\
\hline T506.8 & & & $\begin{array}{l}\text { Ex/9b: c.3306insA } \\
\text { p.Phel I03llefs } X 2\end{array}$ & $\begin{array}{l}\text { I bp insertion } \\
\text { (FS) }\end{array}$ & \\
\hline T506.I & & & $\begin{array}{l}\text { Ex22: c.3745_3764del20 } \\
\text { p.Ser I 249ThrfsX7 }\end{array}$ & $\begin{array}{l}20 \text { bp deletion } \\
\text { (FS) }\end{array}$ & \\
\hline T506.9 & & & $\begin{array}{l}\text { Ex33: c.6364dell I4 } \\
\text { p.Glu2 I22 (through splice site) }\end{array}$ & $\begin{array}{l}\text { II } 4 \text { bp } \\
\text { deletion (FS) }\end{array}$ & \\
\hline T506.6 & & & Ex40: c.7|27-3T > G & Splice site & \\
\hline
\end{tabular}


Table S2. Continued

\begin{tabular}{|c|c|c|c|c|c|}
\hline $\begin{array}{l}\text { Patient } \\
\text { ID }\end{array}$ & $\begin{array}{l}\text { Germline point } \\
\text { mutation }\end{array}$ & $\begin{array}{l}\text { Type of } \\
\text { germline } \\
\text { mutation }\end{array}$ & $\begin{array}{l}\text { Somatic point } \\
\text { mutation }\end{array}$ & $\begin{array}{l}\text { Effect of } \\
\text { somatic } \\
\text { mutation }\end{array}$ & Source \\
\hline TI06.3 & Ex37: c.679linsA p.Tyr2264Xfs & $\begin{array}{l}\text { I bp insertion } \\
\text { (FS) }\end{array}$ & $\begin{array}{l}\text { ExI3: c.2033delC } \\
\text { p.Pro678GInfsX9 R }\end{array}$ & $\begin{array}{l}\text { I bp deletion } \\
\text { (FS) }\end{array}$ & $\begin{array}{l}\text { Unpublished data, } \\
\text { Cardiff }\end{array}$ \\
\hline TI06.4 & & & $\begin{array}{l}\text { Ex26: c. } 4374 \_4375 \text { delCC } \\
\text { p.Leul } 459 \times \underline{R}\end{array}$ & $\begin{array}{l}2 \text { bp deletion } \\
\text { (FS) }\end{array}$ & \\
\hline TI75.I & $\begin{array}{l}\text { Ex37: c. } 6792 C>G \\
\text { p.Tyr } 2264 X\end{array}$ & $\begin{array}{l}\text { Recurrent } \\
\text { nonsense } \\
\text { mutation that } \\
\text { causes a } \\
\text { splicing defect }\end{array}$ & $\begin{array}{l}\text { ExI2a: c. I738insT } \\
\text { p.Tyr580LeufsX7 R }\end{array}$ & $\begin{array}{l}\text { I bp insertion } \\
\text { (FS) }\end{array}$ & $\begin{array}{l}\text { Unpublished data, } \\
\text { Cardiff }\end{array}$ \\
\hline TI43.2 & & & $\begin{array}{l}\text { Ex|9a: c.3।24delGTAGinsAT } \\
\text { p.Vall 042llefs X } 16\end{array}$ & Indel (FS) & \\
\hline TI43.13 & & & $\begin{array}{l}\text { Ex30: c.573I delT } \\
\text { p.Ser I9IILeufs X9 R }\end{array}$ & $\begin{array}{l}\text { I bp deletion } \\
\text { (FS) }\end{array}$ & \\
\hline TI75.2A & & & $\begin{array}{l}\text { Ex3I: c. } 5817 C>A \\
\text { p.Cys1939X } \underline{R}\end{array}$ & Nonsense & \\
\hline T54I.3 & $\begin{array}{l}\text { Ex40: c.7I } 27 \text {. } 7258 \text { del } 132 \\
\text { p.Gly2376. Is this a complete } \\
\text { exon } 40 \text { deletion?? }\end{array}$ & $\begin{array}{l}\text { I } 32 \text { bp In- } \\
\text { frame deletion } \\
\text { (FS) Complete } \\
\text { exon } 40 \\
\text { deletion?? }\end{array}$ & $\begin{array}{l}\text { ExI2b: c. I888delG } \\
\text { p.Val630X } \underline{R}\end{array}$ & $\begin{array}{l}\text { I bp deletion } \\
\text { (FS) }\end{array}$ & $\begin{array}{l}\text { Unpublished data, } \\
\text { Cardiff }\end{array}$ \\
\hline T54I.I & & & $\begin{array}{l}\text { Ex27b: c.4743insG } \\
\text { p.Asp I 582Glufs X18 }\end{array}$ & $\begin{array}{l}\text { I bp insertion } \\
\text { (FS) }\end{array}$ & \\
\hline T536B & & & $\begin{array}{l}\text { Ex40: c.7169delG } \\
\text { p.Arg2390LysfsX6 }\end{array}$ & $\begin{array}{l}\text { I bp deletion } \\
\text { (FS) }\end{array}$ & \\
\hline T210.I & $\begin{array}{l}\text { Ex42: c.7458delC } \\
\text { p.Tyr2487Ilefs }\end{array}$ & $\begin{array}{l}\text { I bp deletion } \\
\text { (FS) }\end{array}$ & Ex7: c. $1062+I G>A \underline{R}$ & Splice site & $\begin{array}{l}\text { Unpublished data, } \\
\text { Cardiff }\end{array}$ \\
\hline T210.6 & & & Ex22: c. $3870+2 T>A$ & Splice site & \\
\hline TI8I.3 & $\begin{array}{l}\text { E6-27b: Partial deletion of } \\
\text { gene } 90 \mathrm{~kb}\end{array}$ & $\begin{array}{l}\text { Partial gene } \\
\text { deletion }\end{array}$ & $\begin{array}{l}\text { Ex3: c.227insG } \\
\text { p.Glu76Glyfs X30 }\end{array}$ & $\begin{array}{l}\text { I bp insertion } \\
\text { (FS) }\end{array}$ & $\begin{array}{l}\text { Unpublished data, } \\
\text { Cardiff }\end{array}$ \\
\hline
\end{tabular}


Table S2. Continued

\begin{tabular}{|c|c|c|c|c|c|}
\hline $\begin{array}{l}\text { Patient } \\
\text { ID }\end{array}$ & $\begin{array}{l}\text { Germline point } \\
\text { mutation }\end{array}$ & $\begin{array}{l}\text { Type of } \\
\text { germline } \\
\text { mutation }\end{array}$ & $\begin{array}{l}\text { Somatic point } \\
\text { mutation }\end{array}$ & $\begin{array}{l}\text { Effect of } \\
\text { somatic } \\
\text { mutation }\end{array}$ & Source \\
\hline $\mathrm{T} 211.2$ & & & $\begin{array}{l}\text { Ex7: c.910C > T } \\
\text { p.Arg304X } \underline{R}\end{array}$ & Nonsense & \\
\hline $\mathrm{T} 211.3$ & & & $\begin{array}{l}\text { Ex17: c } 2855 T>A \\
\text { p.Leu952X }\end{array}$ & Nonsense & \\
\hline T34.I & & & $\begin{array}{l}\text { Ex23.2: c } 4108 \mathrm{C}>\mathrm{T} \\
\text { p.Gln } 1370 \mathrm{X}\end{array}$ & Nonsense & \\
\hline TI50.2 & & & $\begin{array}{l}\text { Ex34: c.6410delT } \\
\text { p.Leu2 } 137 \text { Tyrfs X40 }\end{array}$ & $\begin{array}{l}\text { I bp deletion } \\
\text { (FS) }\end{array}$ & \\
\hline TI8I.I & & & $\begin{array}{l}\text { Ex34: c.6409_64I0delTT } \\
\text { p.Leu2137ThrfsX19 }\end{array}$ & $\begin{array}{l}2 \text { bp deletion } \\
(\mathrm{FS})\end{array}$ & \\
\hline T198 & & & $\begin{array}{l}\text { Ex42: c.7449delT } \\
\text { p.Ala2484GInfs X18 }\end{array}$ & $\begin{array}{l}\text { I bp deletion } \\
\text { (FS) }\end{array}$ & \\
\hline CI76_3 & NFI microdeletion & $\begin{array}{l}\text { Genomic } \\
\text { deletion }\end{array}$ & Ex4a: c. $479+$ IG $>$ A & Splice site & 2 \\
\hline $\mathrm{Cl} 74$ & & & $\begin{array}{l}\text { Ex|5: c.2326- ?.2409 } \\
\text { Complete exon } 15 \text { deletion? }\end{array}$ & $\begin{array}{l}\text { Exon } \\
\text { deletion? }\end{array}$ & \\
\hline $\mathrm{Cl} 86$ & & & ExI7: c. $2990+I G>A \underline{R}$ & Splice site & \\
\hline Cl76_I & & & $\begin{array}{l}\text { Ex28: c. } 48 \mid 2 C>G \\
\text { p.Tyr } 1604 \times \underline{R}\end{array}$ & Nonsense & \\
\hline Cl76_2 & & & $\begin{array}{l}\text { Ex31: c.5927G }>A \\
\text { p.Trp 1976X } \underline{R}\end{array}$ & Nonsense & \\
\hline L-00I D & NFI microdeletion & $\begin{array}{l}\text { Genomic } \\
\text { deletion }\end{array}$ & $\begin{array}{l}\text { Ex4a: c.396_403del8 } \\
\text { p.Leul 34Phefs X2I }\end{array}$ & $\begin{array}{l}8 \text { bp deletion } \\
\text { (FS) }\end{array}$ & 3 \\
\hline L-00I B & & & $\begin{array}{l}\text { Ex 19a: c.3189T >A } \\
\text { p.Cys 1063X }\end{array}$ & Nonsense & \\
\hline L-00I E & & & $\begin{array}{l}\text { Ex22: c.3774G > A } \\
\text { p.TrpI } 258 X\end{array}$ & Nonsense & \\
\hline
\end{tabular}


Table S2. Continued

$\begin{array}{lllll}\text { Patient } & \text { Germline point } & \text { Type of } & \text { Somatic point } & \text { Source } \\ \text { ID } & \text { mutation } & \text { germline } & \text { mutation } & \text { Effect of } \\ & & \text { mutation } & & \text { somatic } \\ \text { mutation }\end{array}$

\begin{tabular}{|c|c|c|c|c|c|}
\hline L-00I C & & & $\begin{array}{l}\text { Ex23.2: c.4086_4092del7 } \\
\text { p.Arg I 362Alafs X20 }\end{array}$ & $\begin{array}{l}7 \text { bp deletion } \\
\text { (FS) }\end{array}$ & \\
\hline L-00I A & & & $\begin{array}{l}\text { Ex28: c.5026_5032del7 } \\
\text { p.Leul 676Alafs I0 }\end{array}$ & $\begin{array}{l}\text { I7 bp deletion } \\
\text { (FS) }\end{array}$ & \\
\hline NF96-I E & NFI microdeletion & $\begin{array}{l}\text { Genomic } \\
\text { deletion }\end{array}$ & $\begin{array}{l}\text { ExI3: c. } 2050 \mathrm{C}>\mathrm{T} \\
\text { p.Glu684X }\end{array}$ & Nonsense & 3 \\
\hline NF96-I B & & & $\begin{array}{l}\text { Ex20: c.3330delT } \\
\text { p.Phe I I IOLeufs X2 }\end{array}$ & $\begin{array}{l}\text { I bp deletion } \\
\text { (FS) }\end{array}$ & \\
\hline NF96-I A & & & Ex4I: c.7394 + IG >A & Splice site & \\
\hline NF96-I C & & & $\begin{array}{l}\text { Ex42: c.7438delG } \\
\text { p.Glu2480Lysfs X22 }\end{array}$ & $\begin{array}{l}\text { I bp deletion } \\
\text { (FS) }\end{array}$ & \\
\hline $\begin{array}{l}\text { NF339- } \\
\text { UHG B }\end{array}$ & NFI microdeletion & & Ex3: c. $288+2 \mathrm{~T}>\mathrm{G}$ & Splice site & 3 \\
\hline $\begin{array}{l}\text { NF339- } \\
\text { UHG C }\end{array}$ & & & $\begin{array}{l}\text { Ex7: c. } 1007 G>A \\
\text { p.Trp336X }\end{array}$ & Nonsense & \\
\hline $\begin{array}{l}\text { NF339- } \\
\text { UHG D }\end{array}$ & & & ExI5: c. $2409+$ IG $>A$ & Splice site & \\
\hline $\begin{array}{l}\text { NF339- } \\
\text { UHG A }\end{array}$ & & & $\begin{array}{l}\text { Ex27b: c. } 4697 \mathrm{~T}>\mathrm{A} \\
\text { p.Leul } 566 \mathrm{X}\end{array}$ & Nonsense & \\
\hline T49.2 & ExI-42: gene deletion & $\begin{array}{l}\text { El-42: gene } \\
\text { deletion }\end{array}$ & $\begin{array}{l}\text { Ex8: c. } 1177 \mathrm{C}>\mathrm{G} \\
\text { p.His393Asp }\end{array}$ & Missense & $\begin{array}{l}\text { Unpublished data, } \\
\text { Cardiff }\end{array}$ \\
\hline T49.8 & & & $\begin{array}{l}\text { Ex8: c. II } 178 \mathrm{~A}>\mathrm{T} \\
\text { p.His393Leu }\end{array}$ & Missense & \\
\hline T49.1 & & & $\begin{array}{l}\text { Ex8: c.II8I_II82delTT } \\
\text { p.Phe394X }\end{array}$ & $\begin{array}{l}2 \text { bp deletion } \\
\text { (FS) }\end{array}$ & \\
\hline T49.5 & & & $\begin{array}{l}\text { ExI6: c. } 2446 C>T \\
\text { p.Arg8I6X } \underline{R}\end{array}$ & Nonsense & \\
\hline
\end{tabular}


Table S2. Continued

\begin{tabular}{|c|c|c|c|c|c|}
\hline $\begin{array}{l}\text { Patient } \\
\text { ID }\end{array}$ & $\begin{array}{l}\text { Germline point } \\
\text { mutation }\end{array}$ & $\begin{array}{l}\text { Type of } \\
\text { germline } \\
\text { mutation }\end{array}$ & $\begin{array}{l}\text { Somatic point } \\
\text { mutation }\end{array}$ & $\begin{array}{l}\text { Effect of } \\
\text { somatic } \\
\text { mutation }\end{array}$ & Source \\
\hline T49.7 & & & $\begin{array}{l}\text { ExI7: c. } 2953 C>T \\
\text { p.Gln } 985 X\end{array}$ & Nonsense & \\
\hline T49.3 & & & $\begin{array}{l}\text { Ex24: c.4I||4_4l|5delGT } \\
\text { p.Val। } 372 X\end{array}$ & $\begin{array}{l}2 \text { bp deletion } \\
\text { (FS) }\end{array}$ & \\
\hline T51.3 & Whole gene deletion & $\begin{array}{l}\text { Genomic } \\
\text { deletion }\end{array}$ & Ex7: c. $1062+$ IG $>$ A $\underline{R}$ & Splice site & $\begin{array}{l}\text { Unpublished data, } \\
\text { Cardiff }\end{array}$ \\
\hline T51.6 & & & $\begin{array}{l}\text { Ex8: c.I179_II80delCT } \\
\text { p.Phe394LeufsX18 }\end{array}$ & $\begin{array}{l}2 \text { bp deletion } \\
\text { (FS) }\end{array}$ & \\
\hline T51.5 & & & $\begin{array}{l}\text { ExII: c.1645_1646delCT } \\
\text { p.Leu549AlafsXI }\end{array}$ & $\begin{array}{l}2 \text { bp deletion } \\
\text { (FS) }\end{array}$ & \\
\hline T5I.4 & & & $\begin{array}{l}\text { Exl6: c.2464G > T } \\
\text { p.Gly822X }\end{array}$ & Nonsense & \\
\hline T51.7 & & & $\begin{array}{l}\text { Ex4l: c.7285C }>T \\
\text { p.Arg2429X } \underline{R}\end{array}$ & Nonsense & \\
\hline TI76.3 & Large deletion & $\begin{array}{l}\text { Genomic } \\
\text { deletion }\end{array}$ & Ex23.2: c.4II0 + IG >C & Splice site & $\begin{array}{l}\text { Unpublished data, } \\
\text { Cardiff }\end{array}$ \\
\hline TI76.I & & & $\begin{array}{l}\text { Ex28: c. } 48 I 2 C>G \\
\text { p. Tyr } 604 X \underline{R}\end{array}$ & Nonsense & \\
\hline T176.2 & & & $\begin{array}{l}\text { Ex3I: c.5928G }>A \\
\text { p.Trp1976X R }\end{array}$ & Nonsense & \\
\hline T217 & ExI: c.6I-IG >C & Splice site & $\begin{array}{l}\text { Ex|2b: c.1900_1907del8 } \\
\text { p.lle634X }\end{array}$ & $\begin{array}{l}8 \text { bp deletion } \\
\text { (FS) }\end{array}$ & $\begin{array}{l}\text { Unpublished data, } \\
\text { Cardiff }\end{array}$ \\
\hline TI440 & $\begin{array}{l}\text { Ex3: c.264_267delTACA } \\
\text { p.Thr89Trpfs }\end{array}$ & $\begin{array}{l}4 \text { bp deletion } \\
\text { (FS) }\end{array}$ & Ex3: c.27IG > A p.Glu9ILys & Missense & $\begin{array}{l}\text { Unpublished data, } \\
\text { Cardiff }\end{array}$ \\
\hline T183.1 & $\begin{array}{l}\text { Ex4a: c.373delGinsATGTGT } \\
\text { p.Arg } 125 \mathrm{fs}\end{array}$ & Indel (FS) & $\begin{array}{l}\text { Ex42: c.7449_7458del10 } \\
\text { p.Leu2483llefsXI5 }\end{array}$ & $\begin{array}{l}10 \text { bp deletion } \\
\text { (FS) }\end{array}$ & $\begin{array}{l}\text { Unpublished data, } \\
\text { Cardiff }\end{array}$ \\
\hline TI39 & $\begin{array}{l}\text { Ex4a: c.434_435delTC } \\
\text { p.Leul45Glufs X19 }\end{array}$ & $\begin{array}{l}2 \text { bp deletion } \\
\text { (FS) }\end{array}$ & $\begin{array}{l}\text { Ex27a: c. } 4637 C>G \\
\text { p.Ser1546X }\end{array}$ & Nonsense & $\begin{array}{l}\text { Unpublished data, } \\
\text { Cardiff }\end{array}$ \\
\hline
\end{tabular}


Table S2. Continued

\begin{tabular}{|c|c|c|c|c|c|}
\hline $\begin{array}{l}\text { Patient } \\
\text { ID }\end{array}$ & $\begin{array}{l}\text { Germline point } \\
\text { mutation }\end{array}$ & $\begin{array}{l}\text { Type of } \\
\text { germline } \\
\text { mutation }\end{array}$ & $\begin{array}{l}\text { Somatic point } \\
\text { mutation }\end{array}$ & $\begin{array}{l}\text { Effect of } \\
\text { somatic } \\
\text { mutation }\end{array}$ & Source \\
\hline TI08.12 & Ex7: c.889-2A > G & Splice site & Ex7: c.910C > T p.Arg304X $\underline{R}$ & Nonsense & 25 \\
\hline TI99.1 & $\begin{array}{l}\text { Ex7: c.983_984delGT } \\
\text { p.Cys328Xfs }\end{array}$ & $\begin{array}{l}2 \text { bp deletion } \\
\text { (FS) }\end{array}$ & $\begin{array}{l}\text { Ex4b: c.528T >A } \\
\text { p.Aspl } 76 \mathrm{Glu}\end{array}$ & Missense & $\begin{array}{l}\text { Unpublished data, } \\
\text { Cardiff }\end{array}$ \\
\hline T374.5 & $\begin{array}{l}\text { ExI0a: c. } 1318 \mathrm{C}>\mathrm{T} \\
\text { p.Arg440X }\end{array}$ & Nonsense & $\begin{array}{l}\text { Ex23.I: c.3916C > T } \\
\text { p.Argl 306X } \underline{R}\end{array}$ & Nonsense & $\begin{array}{l}\text { Unpublished data, } \\
\text { Cardiff }\end{array}$ \\
\hline T996 & ExI0b: c.1393-32T > C & Splice site & Ex6: c.73I-II T>G & Splice site & $\begin{array}{l}\text { Unpublished data, } \\
\text { Cardiff }\end{array}$ \\
\hline T227.3 & $\begin{array}{l}\text { ExI0b: c. I423insC } \\
\text { p.Leu475Profs X9 }\end{array}$ & $\begin{array}{l}\text { I bp insertion } \\
\text { (FS) }\end{array}$ & Ex|5: c.2326-I2C > T & Splice site & $\begin{array}{l}\text { Unpublished data, } \\
\text { Cardiff }\end{array}$ \\
\hline TI6I.4 & $\begin{array}{l}\text { ExI0b: c. I466A > G } \\
\text { p.Tyr } 489 \text { Cys }\end{array}$ & Missense & ExI7: c.2990 + IG > A $\underline{R}$ & Splice site & $\begin{array}{l}\text { Unpublished data, } \\
\text { Cardiff }\end{array}$ \\
\hline TI6I.3 & $\begin{array}{l}\text { ExI0b: c. } 1466 \mathrm{~A}>\mathrm{G} \\
\text { p.Tyr489Cys }\end{array}$ & Missense & $\begin{array}{l}\text { Ex22 : c. } 372 \text { I ins C } \\
\text { p.Arg I } 24 \text { IProfs } X 7\end{array}$ & $\begin{array}{l}\text { I bp insertion } \\
\text { (FS) }\end{array}$ & $\begin{array}{l}\text { Unpublished data, } \\
\text { Cardiff }\end{array}$ \\
\hline T2I4 & ExI0b complete exon deletion & $\begin{array}{l}\text { Single exon } \\
\text { deletion }\end{array}$ & $\begin{array}{l}\text { Ex22: c.3826C > T } \\
\text { p.Arg| } 276 X\end{array}$ & Nonsense & $\begin{array}{l}\text { Unpublished data, } \\
\text { Cardiff }\end{array}$ \\
\hline CLJ8N & ExI3: c.204IC > T p.Arg68IX & Nonsense & ExI3: c.2246C > G p.Ser749X & Nonsense & 6,7 \\
\hline TI70.IA & ExI3: c.204IC > T p.Arg68IX & Nonsense & $\begin{array}{l}\text { Ex|2a: c. } 1797 \mathrm{G}>\mathrm{A} \\
\text { p.Trp599X }\end{array}$ & Nonsense & $\begin{array}{l}\text { Unpublished data, } \\
\text { Cardiff }\end{array}$ \\
\hline TI243 & $\begin{array}{l}\text { Ex|3: c.2197_22|4dell7 } \\
\text { p.Pro733fs }\end{array}$ & $\begin{array}{l}\text { I } 7 \text { bp deletion } \\
\text { (FS) }\end{array}$ & $\begin{array}{l}\text { Ex36: c. } 6709 \mathrm{C}>\mathrm{T} \\
\text { p.Arg2237X }\end{array}$ & Nonsense & $\begin{array}{l}\text { Unpublished data, } \\
\text { Cardiff }\end{array}$ \\
\hline $\begin{array}{l}\text { NF253- } \\
\text { UHG D }\end{array}$ & Exl6: c. $2850+2 A>G$ & Splice site & $\begin{array}{l}\text { ExII: c.1663_1666delTTAG } \\
\text { p.Leu555llefs X12 }\end{array}$ & $\begin{array}{l}4 \text { bp deletion } \\
\text { (FS) }\end{array}$ & 3 \\
\hline TI93 & $\begin{array}{l}\text { ExI7: c.2870delA } \\
\text { p.Asp957llefs }\end{array}$ & $\begin{array}{l}\text { I bp deletion } \\
\text { (FS) }\end{array}$ & $\begin{array}{l}\text { Ex|Oa: c. }|3| 2 G>T \\
\text { p.Glu438X }\end{array}$ & Nonsense & $\begin{array}{l}\text { Unpublished data, } \\
\text { Cardiff }\end{array}$ \\
\hline L-004 D & $\begin{array}{l}\text { Ex|8: c.3।I3G >A } \\
\text { p.Argl038Lys }\end{array}$ & Missense & $\begin{array}{l}\text { Ex27b: c.4729delA } \\
\text { p.Thr I577Leufs X23 }\end{array}$ & $\begin{array}{l}\text { I bp deletion } \\
\text { (FS) }\end{array}$ & 3 \\
\hline HTI359.2 & Ex|8: c.3III + IG >A & Splice site & $\begin{array}{l}\text { ExI0a: c. } 1277 G>A \\
\text { p.Trp426X R }\end{array}$ & Nonsense & $\begin{array}{l}\text { Unpublished data, } \\
\text { Cardiff }\end{array}$ \\
\hline
\end{tabular}


Table S2. Continued

\begin{tabular}{|c|c|c|c|c|c|}
\hline $\begin{array}{l}\text { Patient } \\
\text { ID }\end{array}$ & $\begin{array}{l}\text { Germline point } \\
\text { mutation }\end{array}$ & $\begin{array}{l}\text { Type of } \\
\text { germline } \\
\text { mutation }\end{array}$ & $\begin{array}{l}\text { Somatic point } \\
\text { mutation }\end{array}$ & $\begin{array}{l}\text { Effect of } \\
\text { somatic } \\
\text { mutation }\end{array}$ & Source \\
\hline TI40.4 & $\begin{array}{l}\text { Ex22: c.3732delT } \\
\text { p.Thr|245LeufsX2I }\end{array}$ & $\begin{array}{l}\text { I bp deletion } \\
\text { (FS) }\end{array}$ & $\begin{array}{l}\text { Ex4l: c.7285C }>T \\
\text { p.Arg2429X } \underline{R}\end{array}$ & Nonsense & 25 \\
\hline T37.I & $\begin{array}{l}\text { Ex23.2: c. } 4084 C>T \\
\text { p.Argl } 362 X\end{array}$ & Nonsense & $\begin{array}{l}\text { Exl0b:c.1467T > G } \\
\text { p. Tyr } 489 X\end{array}$ & Nonsense & $\begin{array}{l}\text { Unpublished data, } \\
\text { Cardiff }\end{array}$ \\
\hline T205.1 & $\begin{array}{l}\text { Ex24: c. } 4196 C>A \\
\text { p.Serl399X }\end{array}$ & Nonsense & $\begin{array}{l}\text { Ex27a: c.4537C }>T \\
\text { p.Arg15।3X } \underline{R}\end{array}$ & Nonsense & $\begin{array}{l}\text { Unpublished data, } \\
\text { Cardiff }\end{array}$ \\
\hline T450.3 & $\begin{array}{l}\text { Ex27a: c. } 4537 C>T \\
\text { p.Arg } 1513 X\end{array}$ & Nonsense & $\begin{array}{l}\text { Ex4b: c. } 574 C>T \\
\text { p.Argl } 92 \times R\end{array}$ & Nonsense & $\begin{array}{l}\text { Unpublished data, } \\
\text { Cardiff }\end{array}$ \\
\hline T209.8 & $\begin{array}{l}\text { Ex:28: c. } 4950 \mathrm{C}>\mathrm{G} \\
\text { p.Tyr1650X }\end{array}$ & Nonsense & $\begin{array}{l}\text { ExI0a: c. } 1318 \mathrm{C}>\mathrm{T} \\
\text { p.Arg440X R }\end{array}$ & Nonsense & $\begin{array}{l}\text { Unpublished data, } \\
\text { Cardiff }\end{array}$ \\
\hline $\begin{array}{l}\text { NFII6- } \\
\text { UHG A }\end{array}$ & $\begin{array}{l}\text { Ex28: c.5I22insG } \\
\text { p.Ala I708GlyfsX27 }\end{array}$ & $\begin{array}{l}\text { I bp insertion } \\
\text { (FS) }\end{array}$ & $\begin{array}{l}\text { Ex27a: c.4537C }>T \\
\text { p.Arg1513X R }\end{array}$ & Nonsense & 3 \\
\hline TI308 & Ex29: c.5546 + 19 T>A & Splice site & $\begin{array}{l}\text { Ex22: c.3827G }>A \\
\text { p.Arg } 1276 \mathrm{Gln}\end{array}$ & Missense & $\begin{array}{l}\text { Unpublished data, } \\
\text { Cardiff }\end{array}$ \\
\hline T149.5C & $\begin{array}{l}\text { Ex34: } \\
\text { c. } 65 \text { I } 2 \text { delATGAGAGAinsC } \\
\text { p.Tyr } 217 \mathrm{Ifs}\end{array}$ & Indel (FS) & $\begin{array}{l}\text { Ex7: c.988G }>A \\
\text { p.Ala330Thr }\end{array}$ & Missense & $\begin{array}{l}\text { Unpublished data, } \\
\text { Cardiff }\end{array}$ \\
\hline T89.1 & $\begin{array}{l}\text { Ex37: c.6789_6792delTTAC } \\
\text { p.Asp2264Thrfs X5 }\end{array}$ & $\begin{array}{l}4 \text { bp deletion } \\
\text { (FS) }\end{array}$ & $\begin{array}{l}\text { ExI2b: c. 1888delG } \\
\text { p.Val630X } \underline{R}\end{array}$ & $\begin{array}{l}\text { I bp deletion } \\
\text { (FS) }\end{array}$ & 25 \\
\hline T106.I & $\begin{array}{l}\text { Ex37: c.679linsA } \\
\text { p.Tyr2264Xfs XI }\end{array}$ & $\begin{array}{l}\text { I bp insertion } \\
\text { (FS) }\end{array}$ & $\begin{array}{l}\text { Ex13: c.2033delC } \\
\text { p.Pro678Glnfs X9 R }\end{array}$ & $\begin{array}{l}\text { I bp deletion } \\
\text { (FS) }\end{array}$ & 25 \\
\hline L-004 B & $\begin{array}{l}\text { Ex37: c.679linsA } \\
\text { p.Tyr2264XfsXI }\end{array}$ & $\begin{array}{l}\text { I bp insertion } \\
\text { (FS) }\end{array}$ & $\begin{array}{l}\text { Ex23.I: c.387I_3974dell } 03 \\
\text { Complete exon } 23.1 \text { deletion? }\end{array}$ & $\begin{array}{l}103 \mathrm{bp} \\
\text { deletion (FS) }\end{array}$ & 3 \\
\hline TI200 & $\begin{array}{l}\text { Ex37: c.679linsA } \\
\text { p.Tyr2264XfsXI }\end{array}$ & $\begin{array}{l}\text { I bp insertion } \\
\text { (FS) }\end{array}$ & $\begin{array}{l}\text { Ex16: c.2825G > T } \\
\text { p.Ser942lle }\end{array}$ & Missense & $\begin{array}{l}\text { Unpublished data, } \\
\text { Cardiff }\end{array}$ \\
\hline CLOIN & $\begin{array}{l}\text { Ex37: c. } 6792 C>A \\
\text { p.Tyr2264X }\end{array}$ & Nonsense & mRNA study: Exon 4c skipped & Splice site? & 6,7 \\
\hline
\end{tabular}


Table S2. Continued

\begin{tabular}{|c|c|c|c|c|c|}
\hline $\begin{array}{l}\text { Patient } \\
\text { ID }\end{array}$ & $\begin{array}{l}\text { Germline point } \\
\text { mutation }\end{array}$ & $\begin{array}{l}\text { Type of } \\
\text { germline } \\
\text { mutation }\end{array}$ & $\begin{array}{l}\text { Somatic point } \\
\text { mutation }\end{array}$ & $\begin{array}{l}\text { Effect of } \\
\text { somatic } \\
\text { mutation }\end{array}$ & Source \\
\hline TI229 & $\begin{array}{l}\text { Ex39: c.7049_7064dell6 } \\
\text { p.Cys2350PhefsX19 }\end{array}$ & $\begin{array}{l}16 \text { bp deletion } \\
\text { (FS) }\end{array}$ & $\begin{array}{l}\text { ExI3: c. } 2203 \mathrm{~T}>\mathrm{C} \\
\text { p.Tyr735His }\end{array}$ & Missense & $\begin{array}{l}\text { Unpublished data, } \\
\text { Cardiff }\end{array}$ \\
\hline TI64.IE & $\begin{array}{l}\text { Ex4l: c. } 7285 C>T \\
\text { p.Arg2429X }\end{array}$ & Nonsense & $\begin{array}{l}\text { Ex23.2: c. } 4084 C>T \\
\text { p.Argl } 362 \times \underline{R}\end{array}$ & Nonsense & $\begin{array}{l}\text { Unpublished data, } \\
\text { Cardiff }\end{array}$ \\
\hline TI57.IA & Ex45: c.7907 + 3A > T & Splice site & $\begin{array}{l}\text { Ex20: c.3492delC } \\
\text { p.llel I65SerfsX2 }\end{array}$ & $\begin{array}{l}\text { I bp deletion } \\
\text { (FS) }\end{array}$ & $\begin{array}{l}\text { Unpublished data, } \\
\text { Cardiff }\end{array}$ \\
\hline T98.6 & I.5Mb deletion & $\begin{array}{l}\text { Genomic } \\
\text { deletion }\end{array}$ & $\begin{array}{l}\text { Ex34: c.6387A >C } \\
\text { p.Arg2 } 129 \text { Ser }\end{array}$ & Missense & 25 \\
\hline T98 & Complete gene deletion & $\begin{array}{l}\text { Genomic } \\
\text { deletion }\end{array}$ & $\begin{array}{l}\text { Ex20: c.3457_3460del4 } \\
\text { p.Leul I53MetfsX3 }\end{array}$ & $\begin{array}{l}4 \text { bp deletion } \\
\text { (FS) }\end{array}$ & $\begin{array}{l}\text { Unpublished data, } \\
\text { Cardiff }\end{array}$ \\
\hline TI58.I & Complete gene deletion & $\begin{array}{l}\text { Genomic } \\
\text { deletion }\end{array}$ & $\begin{array}{l}\text { ExI8: c.3058delG } \\
\text { p.Glu 1020LysfsX2 R- }\end{array}$ & $\begin{array}{l}\text { I bp deletion } \\
\text { (FS) }\end{array}$ & $\begin{array}{l}\text { Unpublished data, } \\
\text { Cardiff }\end{array}$ \\
\hline CCFIN & Complete gene deletion & $\begin{array}{l}\text { Genomic } \\
\text { deletion }\end{array}$ & $\begin{array}{l}\text { mRNA study: exons } 12 \mathrm{a} \text { and } \\
12 \mathrm{~b} \text { skipped }\end{array}$ & Splice site? & 5,6 \\
\hline $\begin{array}{l}\text { UWAI28- } \\
3\end{array}$ & $\mathrm{NI}$ & $\mathrm{NI}$ & $\begin{array}{l}\text { Ex4b: c.543_546delGTAT } \\
\text { p.Tyr I82SerfsX7 }\end{array}$ & $\begin{array}{l}4 \text { bp deletion } \\
\text { (FS) }\end{array}$ & 38 \\
\hline T219.1 & $\mathrm{NI}$ & $\mathrm{NI}$ & $\begin{array}{l}\text { Ex9: c.1225_1226delGT } \\
\text { p.Val409AlafsX18 }\end{array}$ & $\begin{array}{l}2 \text { bp deletion } \\
\text { (FS) }\end{array}$ & $\begin{array}{l}\text { Unpublished data, } \\
\text { Cardiff }\end{array}$ \\
\hline TII6 & $\mathrm{NI}$ & $\mathrm{NI}$ & $\begin{array}{l}\text { ExI0c: c.I54I_I542delAG } \\
\text { p.G } \ln 5 \text { I } 4 \text { Argfs X43 }\end{array}$ & $\begin{array}{l}2 \text { bp deletion } \\
\text { (FS) }\end{array}$ & 25 \\
\hline TI98.I & $\mathrm{NI}$ & $\mathrm{NI}$ & $\begin{array}{l}\text { ExI0c: c. } 1555 \mathrm{C}>\mathrm{T} \\
\text { p.Gln519X }\end{array}$ & Nonsense & $\begin{array}{l}\text { Unpublished data, } \\
\text { Cardiff }\end{array}$ \\
\hline TI28.17 & $\mathrm{NI}$ & $\mathrm{NI}$ & $\begin{array}{l}\text { ExI0c: c.1556A >C } \\
\text { p.Gln519Pro } \underline{R}\end{array}$ & Missense & 25 \\
\hline T198.2 & $\mathrm{NI}$ & $\mathrm{NI}$ & $\begin{array}{l}\text { ExI2a: c. } 1792 \mathrm{~A}>\mathrm{T} \\
\text { p.Lys598X }\end{array}$ & Nonsense & $\begin{array}{l}\text { Unpublished data, } \\
\text { Cardiff }\end{array}$ \\
\hline T63.2 & $\mathrm{NI}$ & $\mathrm{NI}$ & Ex|3: c.2088delG p.Trp696X & $\begin{array}{l}\text { I bp deletion } \\
\text { (FS) }\end{array}$ & 25 \\
\hline
\end{tabular}


Table S2. Continued

\begin{tabular}{|c|c|c|c|c|c|}
\hline $\begin{array}{l}\text { Patient } \\
\text { ID }\end{array}$ & $\begin{array}{l}\text { Germline point } \\
\text { mutation }\end{array}$ & $\begin{array}{l}\text { Type of } \\
\text { germline } \\
\text { mutation }\end{array}$ & $\begin{array}{l}\text { Somatic point } \\
\text { mutation }\end{array}$ & $\begin{array}{l}\text { Effect of } \\
\text { somatic } \\
\text { mutation }\end{array}$ & Source \\
\hline TI46.5 & $\mathrm{NI}$ & $\mathrm{NI}$ & $\begin{array}{l}\text { ExI5: c. } 2326 G>A \\
\text { p.Ala776Thr } \underline{R}\end{array}$ & $\begin{array}{l}\text { Missense/ } \\
\text { splicing? }\end{array}$ & $\begin{array}{l}\text { Unpublished data, } \\
\text { Cardiff }\end{array}$ \\
\hline T63.8 & $\mathrm{NI}$ & $\mathrm{NI}$ & $\begin{array}{l}\text { ExI5: c.234I_2358dell8 } \\
\text { p.His78IAla (in-frame) }\end{array}$ & $\begin{array}{l}\text { I } 8 \text { bp deletion } \\
\text { (in-frame) }\end{array}$ & 25 \\
\hline TI265.2 & $\mathrm{NI}$ & $\mathrm{NI}$ & ExI7: c.285I-I6T >C & Splice site & $\begin{array}{l}\text { Unpublished data, } \\
\text { Cardiff }\end{array}$ \\
\hline T233.I & $\mathrm{NI}$ & $\mathrm{NI}$ & ExI7: c.2879del38 p.Phe960X & $\begin{array}{l}38 \text { bp deletion } \\
\text { (FS) }\end{array}$ & \\
\hline TI58.2 & $\mathrm{NI}$ & $\mathrm{NI}$ & $\begin{array}{l}\text { ExI8: c.3058delG } \\
\text { p.Glul020LysfsX2 R }\end{array}$ & $\begin{array}{l}\text { I bp deletion } \\
\text { (FS) }\end{array}$ & \\
\hline TI58.4 & $\mathrm{NI}$ & $\mathrm{NI}$ & $\begin{array}{l}\text { ExI8: c.3058delG } \\
\text { p.Glu 1020LysfsX2 R }\end{array}$ & $\begin{array}{l}\text { I bp deletion } \\
\text { (FS) }\end{array}$ & \\
\hline T192.I & $\mathrm{NI}$ & $\mathrm{NI}$ & ExI8: c. $3113+I G>A \underline{R}$ & Splice site & \\
\hline T192.2 & $\mathrm{NI}$ & $\mathrm{NI}$ & $\operatorname{Ex|8:c.31I3+IG>AR}$ & Splice site & \\
\hline NF260-I & $\mathrm{NI}$ & $\mathrm{NI}$ & $\begin{array}{l}\text { Ex22: c. } 372 \mid \mathrm{C}>\mathrm{T} \\
\text { p.ArgI } 24 \mid X \underline{\mathrm{R}}\end{array}$ & Nonsense & 8 \\
\hline 38 & $\mathrm{NI}$ & $\mathrm{NI}$ & $\begin{array}{l}\text { Ex22: c.3727_3728delCT } \\
\text { p.Leu I } 243 G \text { Gyfs X5 }\end{array}$ & $\begin{array}{l}2 \text { bp deletion } \\
\text { (FS) }\end{array}$ & 18 \\
\hline T94 & $\mathrm{NI}$ & $\mathrm{NI}$ & $\begin{array}{l}\text { Ex23.2: c. } 4083 \text { ins } T \\
\text { p.Arg | } 362 \text { Serfs X } 12\end{array}$ & $\begin{array}{l}\text { I bp insertion } \\
\text { (FS) }\end{array}$ & 25 \\
\hline T565 & $\mathrm{NI}$ & NI & Ex25: c. $4270-2 A>G$ & Splice site & $\begin{array}{l}\text { Unpublished data, } \\
\text { Cardiff }\end{array}$ \\
\hline TI06.3 & $\mathrm{NI}$ & $\mathrm{NI}$ & $\begin{array}{l}\text { Ex26: c. } 4374 \_4375 \text { delCC } \\
\text { p.Asp I } 460 \times \underline{R}\end{array}$ & $\begin{array}{l}2 \text { bp deletion } \\
\text { (FS) }\end{array}$ & 25 \\
\hline T8I.I & $\mathrm{NI}$ & $\mathrm{NI}$ & Ex27b: c. $4662-5 C>T$ & Splice site & 25 \\
\hline TI284.5 & $\mathrm{NI}$ & $\mathrm{NI}$ & Ex27b: c. $4772+5 G>A$ & Splice site & $\begin{array}{l}\text { Unpublished data, } \\
\text { Cardiff }\end{array}$ \\
\hline
\end{tabular}


Table S2. Continued

\begin{tabular}{|c|c|c|c|c|c|}
\hline $\begin{array}{l}\text { Patient } \\
\text { ID }\end{array}$ & $\begin{array}{l}\text { Germline point } \\
\text { mutation }\end{array}$ & $\begin{array}{l}\text { Type of } \\
\text { germline } \\
\text { mutation }\end{array}$ & $\begin{array}{l}\text { Somatic point } \\
\text { mutation }\end{array}$ & $\begin{array}{l}\text { Effect of } \\
\text { somatic } \\
\text { mutation }\end{array}$ & Source \\
\hline 20 & $\mathrm{NI}$ & $\mathrm{NI}$ & $\begin{array}{l}\text { Ex33: c.6253_6354 + } 5 \text { dell I } 7 \\
\text { p.Val2085 (through splice site) }\end{array}$ & $\begin{array}{l}\text { I } 7 \text { bp deletion } \\
\text { (FS) }\end{array}$ & 18 \\
\hline 44 & $\mathrm{NI}$ & $\mathrm{NI}$ & $\begin{array}{l}\text { Ex40: c.7I27-44_7I74del92 } \\
\text { p.Gly2376ValfsX8 }\end{array}$ & $\begin{array}{l}92 \text { bp deletion } \\
\text { (FS) }\end{array}$ & 18 \\
\hline \multicolumn{6}{|l|}{ PNFs } \\
\hline 45 & $\begin{array}{l}\text { Ex3: c.264_267delTACA } \\
\text { p.Thr89Trpfs X8 }\end{array}$ & $\begin{array}{l}4 \text { bp deletion } \\
\text { (FS) }\end{array}$ & Ex3: c.27IG > A p.Glu9ILys & Missense & 10 \\
\hline T399 & $\begin{array}{l}\text { Ex3: c.264_267delTACA } \\
\text { p.Thr89TrpfsX8 }\end{array}$ & $\begin{array}{l}4 \text { bp deletion } \\
\text { (FS) }\end{array}$ & Ex3: c.27IG > T p.Glu9IX & Nonsense & $\begin{array}{l}\text { Unpublished data, } \\
\text { Cardiff }\end{array}$ \\
\hline T7 & Ex4a: c. $479+$ IG $>A$ & Splice site & $\begin{array}{l}\text { Exl6: c. } 2446 C>T \\
\text { p.Arg8I6X } \underline{R}\end{array}$ & Nonsense & 39 \\
\hline 19 UK & Ex7: c.910C > T p.Arg304X & Nonsense & $\begin{array}{l}\text { Ex8: c. II77_II78delCA } \\
\text { p.His393Leufs X16 }\end{array}$ & $\begin{array}{l}2 \text { bp deletion } \\
\text { (FS) }\end{array}$ & $\begin{array}{l}\text { Unpublished data, } \\
\text { Cardiff }\end{array}$ \\
\hline c3 UK & Ex8: c. $1063-2 A>G$ & Splice site & Ex7: c.910C > T p.Arg304X $\underline{R}$ & Nonsense & \\
\hline $14 b$ & Ex|3: c. 2076 C > G p.Tyr692X & Nonsense & $\begin{array}{l}\text { Ex4b: c.532_558del27 } \\
\text { p.Glul } 78 \underline{R}\end{array}$ & $\begin{array}{l}27 \text { bp deletion } \\
\text { (in-frame) }\end{array}$ & \\
\hline T318 & Ex13: c.2076C > G p.Tyr692X & Nonsense & $\begin{array}{l}\text { Ex4b: c.532_558del27 } \\
\text { p.GluI78 } \underline{R}\end{array}$ & $\begin{array}{l}27 \text { bp deletion } \\
\text { (in-frame) }\end{array}$ & \\
\hline T38I.I & $E \mid 8: c .31 I 3+I G>A$ & Splice site & $\begin{array}{l}\text { ExI0a: c. } 1277 G>A \\
\text { p.Trp426X } \underline{R}\end{array}$ & Nonsense & \\
\hline T38I.2 & & & ExI8: c. $3113+I G>A \underline{R}$ & Splice site & \\
\hline 31 & $\begin{array}{l}\text { Ex29: c.5234C > G } \\
\text { p.Serl } 745 X\end{array}$ & Nonsense & Ex9: c. I246C > T p.Arg4I6X & Nonsense & \\
\hline c4 UK & $\begin{array}{l}\text { Ex33: c.6289_6290insA } \\
\text { p.Leu2097fs X2 }\end{array}$ & $\begin{array}{l}\text { I bp insertion } \\
\text { (FS) }\end{array}$ & $\begin{array}{l}\text { Ex27b: c. } 4706 \mathrm{~T}>\mathrm{G} \\
\text { p.Leul } 569 \times \underline{\mathrm{R}}\end{array}$ & Nonsense & \\
\hline TI55 & $\begin{array}{l}\text { Ex33: c.629linsA } \\
\text { p.Leu2097Xfs X9 }\end{array}$ & $\begin{array}{l}\text { I bp insertion } \\
\text { (FS) }\end{array}$ & $\begin{array}{l}\text { Ex27b: c. } 4706 \mathrm{~T}>\mathrm{G} \\
\text { p.Leul } 569 \times \underline{\mathrm{R}}\end{array}$ & Nonsense & \\
\hline
\end{tabular}


Table S2. Continued

\begin{tabular}{|c|c|c|c|c|c|}
\hline $\begin{array}{l}\text { Patient } \\
\text { ID }\end{array}$ & $\begin{array}{l}\text { Germline point } \\
\text { mutation }\end{array}$ & $\begin{array}{l}\text { Type of } \\
\text { germline } \\
\text { mutation }\end{array}$ & $\begin{array}{l}\text { Somatic point } \\
\text { mutation }\end{array}$ & $\begin{array}{l}\text { Effect of } \\
\text { somatic } \\
\text { mutation }\end{array}$ & Source \\
\hline 24 & Complete gene deletion & $\begin{array}{l}\text { Genomic } \\
\text { deletion }\end{array}$ & $\begin{array}{l}\text { Ex4b: c. } 528 \mathrm{~T}>\mathrm{A} \\
\text { p.Aspl } 76 \mathrm{Glu}\end{array}$ & Missense & $\begin{array}{l}\text { Unpublished data, } \\
\text { Cardiff }\end{array}$ \\
\hline T323 & $\begin{array}{l}\text { Complete gene deletion } \\
(1.4 \mathrm{Mb} \text { ?) }\end{array}$ & $\begin{array}{l}\text { Genomic } \\
\text { deletion }\end{array}$ & $\begin{array}{l}\text { Ex26: c. } 450 \mathrm{I} \_4502 \text { delCT } \\
\text { p.LeuI50IPhefs X7 } \underline{R}\end{array}$ & $\begin{array}{l}2 \text { bp deletion } \\
\text { (FS) }\end{array}$ & \\
\hline T369 & $\begin{array}{l}\text { Complete gene deletion } \\
(\mathrm{I} .4 \mathrm{Mb} \text { ?) }\end{array}$ & $\begin{array}{l}\text { Genomic } \\
\text { deletion }\end{array}$ & $\begin{array}{l}\text { Ex26: c. } 450 \mathrm{I} \_4502 \mathrm{delCT} \\
\text { p.Leul50IPhefs X7 } \underline{\mathrm{R}}\end{array}$ & $\begin{array}{l}2 \text { bp deletion } \\
\text { (FS) }\end{array}$ & \\
\hline c2 UK & $\mathrm{NI}$ & $\mathrm{NI}$ & $\begin{array}{l}\text { Ex23.2: c. } 4083 \text { insT } \\
\text { p.Arg | 362Serfs X } 12\end{array}$ & $\begin{array}{l}\text { I bp insertion } \\
\text { (FS) }\end{array}$ & $\begin{array}{l}\text { Unpublished data, } \\
\text { Cardiff }\end{array}$ \\
\hline 42 & $\mathrm{NI}$ & $\mathrm{NI}$ & Ex27a: c. $45 I 5-2 A>G$ & Splice site & \\
\hline T329? & $\mathrm{NI}$ & $\mathrm{NI}$ & $\begin{array}{l}\text { Ex7: c.952_953delGA } \\
\text { p.Glu3 I8LysfsXII }\end{array}$ & $\begin{array}{l}2 \text { bp deletion } \\
\text { (FS) }\end{array}$ & \\
\hline \multicolumn{6}{|c|}{ Spinal neurofibromas } \\
\hline I & Ex7: c.899T >C p.Leu300Pro & Missense & Ex24: c. 4||$I-2 A>G$ & Splice site & 16 \\
\hline 13 & I.4 Mb deletion & $\begin{array}{l}\text { Genomic } \\
\text { deletion }\end{array}$ & Ex21_22 splice site mutation? & Splice site? & \\
\hline 6 & I.4 Mb deletion & $\begin{array}{l}\text { Genomic } \\
\text { deletion }\end{array}$ & $\begin{array}{l}\text { Ex27b: c. } 4690 \mathrm{~A}>\mathrm{G} \\
\text { p.Lys I } 564 \mathrm{Glu}\end{array}$ & Missense & \\
\hline \multicolumn{6}{|c|}{ MPNSTs } \\
\hline 53 & Ex4b: c.574C > T p.Arg192X & Nonsense & Ex24: c.4203insT p.Glul 402X & $\begin{array}{l}\text { I bp insertion } \\
\text { (FS) }\end{array}$ & 18 \\
\hline TI68 & Ex5: c.663G > A p.Trp22IX & Nonsense & $\begin{array}{l}\text { Ex34: c.6444delA } \\
\text { p.Val2 I49Serfs X28 }\end{array}$ & $\begin{array}{l}\text { I bp deletion } \\
\text { (FS) }\end{array}$ & \\
\hline TI85 & $\begin{array}{l}\text { Ex6: c.773delA } \\
\text { p.Ser259Alafs X21 }\end{array}$ & $\begin{array}{l}\text { I bp deletion } \\
\text { (FS) }\end{array}$ & $\begin{array}{l}\text { Ex34: c.64I0delT } \\
\text { p.Leu2 I 37Tyrfs X4I }\end{array}$ & $\begin{array}{l}\text { I bp deletion } \\
\text { (FS) }\end{array}$ & \\
\hline 37 & Exl6: c. $2446 C>T$ p.Arg8I6X & Nonsense & $\begin{array}{l}\text { Ex6: c.73I-5_74I del19 } \\
\text { through a splice site }\end{array}$ & $\begin{array}{l}19 \text { bp deletion } \\
\text { (FS) }\end{array}$ & \\
\hline
\end{tabular}


Table S2. Continued

\begin{tabular}{|c|c|c|c|c|c|}
\hline $\begin{array}{l}\text { Patient } \\
\text { ID }\end{array}$ & $\begin{array}{l}\text { Germline point } \\
\text { mutation }\end{array}$ & $\begin{array}{l}\text { Type of } \\
\text { germline } \\
\text { mutation }\end{array}$ & $\begin{array}{l}\text { Somatic point } \\
\text { mutation }\end{array}$ & $\begin{array}{l}\text { Effect of } \\
\text { somatic } \\
\text { mutation }\end{array}$ & Source \\
\hline 17 & $\begin{array}{l}\text { Ex20: c.3457_3460delCTCA } \\
\text { p.Leul I 53MetfsX4 }\end{array}$ & $\begin{array}{l}2 \text { bp deletion } \\
\text { (FS) }\end{array}$ & $\begin{array}{l}\text { Ex3I: c.5789delC } \\
\text { p.Prol } 930 \mathrm{Hisf} \times 6\end{array}$ & $\begin{array}{l}\text { I bp deletion } \\
\text { (FS) }\end{array}$ & 17 \\
\hline 20 & I.4Mb deletion & $\begin{array}{l}\text { Genomic } \\
\text { deletion }\end{array}$ & $\begin{array}{l}\text { ExI0c: c. I532delC } \\
\text { p.Pro5IIGInfsXI4 }\end{array}$ & $\begin{array}{l}\text { I bp deletion } \\
\text { (FS) }\end{array}$ & 17 \\
\hline 44 & Complete gene deletion & $\begin{array}{l}\text { Genomic } \\
\text { deletion }\end{array}$ & $\begin{array}{l}\text { Exl6: c. } 2446 C>T \\
\text { p.Arg8I6X } \underline{R}\end{array}$ & Nonsense & 18 \\
\hline TI84 & Segmental NF NI & $\mathrm{NI}$ & $\begin{array}{l}\text { Ex27a: c.4580_4590dell I } \\
\text { p.Prol527GInfsXII } \underline{R}\end{array}$ & $\begin{array}{l}\text { I I bp deletion } \\
\text { (FS) }\end{array}$ & 18 \\
\hline II & $\mathrm{NI}$ & $\mathrm{NI}$ & $\begin{array}{l}\text { Ex27a: c.4580_4590dell I } \\
\text { p.Prol527GInfsXII } \underline{R}\end{array}$ & $\begin{array}{l}\text { I I bp deletion } \\
\text { (FS) }\end{array}$ & 17 \\
\hline 38 & $\mathrm{NI}$ & $\mathrm{NI}$ & $\begin{array}{l}\text { ExI2a: c. I } 83 \text { I delCinsTT } \\
\text { p.Leu6II IPhefs X3 }\end{array}$ & Indel (FS) & 18 \\
\hline \multicolumn{6}{|l|}{ GISTs } \\
\hline NFI-la & Ex24: c. $4269+$ IG $>T$ & Splice site & Ex29: c. $5546+2 \mathrm{~T}>\mathrm{A}$ & Splice site & 3 \\
\hline $\mathrm{NFI}-\mathrm{Ib}$ & & & $\begin{array}{l}\text { Ex29: c. } 5242 C>T \\
\text { p.Arg I } 748 \times \underline{R}\end{array}$ & Nonsense & \\
\hline NFI-2a & Ex37: c.679linsA p.Tyr2264X & $\begin{array}{l}\text { Ibp insertion } \\
\text { (FS) }\end{array}$ & $\begin{array}{l}\text { Ex3: c. } 279 \mathrm{~T}>\mathrm{A} \\
\text { p.Cys } 93 \mathrm{X}\end{array}$ & Nonsense & 3 \\
\hline NFI-2c & & & ExI0c: c. del2I & $\begin{array}{l}21 \text { bp in-frame } \\
\text { deletion }\end{array}$ & \\
\hline$N F I-2 b$ & & & $\begin{array}{l}\text { Ex45: c.7846C > T } \\
\text { p.Arg26I6X }\end{array}$ & Nonsense & \\
\hline
\end{tabular}


Table S2. Continued

\begin{tabular}{|c|c|c|c|c|c|}
\hline $\begin{array}{l}\text { Patient } \\
\text { ID }\end{array}$ & $\begin{array}{l}\text { Germline point } \\
\text { mutation }\end{array}$ & $\begin{array}{l}\text { Type of } \\
\text { germline } \\
\text { mutation }\end{array}$ & $\begin{array}{l}\text { Somatic point } \\
\text { mutation }\end{array}$ & $\begin{array}{l}\text { Effect of } \\
\text { somatic } \\
\text { mutation }\end{array}$ & Source \\
\hline \multicolumn{6}{|l|}{ JMML } \\
\hline DI 27 & $\begin{array}{l}\text { Ex|4: } \\
\text { c.2288_2295dupTGAGGCGC } \\
\text { / Ex20: c.3366delT }\end{array}$ & $\begin{array}{l}\text { Compound } \\
\text { heterozygous } \\
\mathrm{NFImutations} \\
\text { found in } \\
\text { blood cells }\end{array}$ & $\begin{array}{l}\text { Ex|4: } \\
\text { c.2288_2295dupTGAGGCGC } \\
\text { / Ex20: c.3366delT }\end{array}$ & $\begin{array}{l}\text { Compound } \\
\text { heterozygous } \\
\mathrm{NFI} \text { mutations } \\
\text { found in } \\
\text { blood cells }\end{array}$ & 31 \\
\hline CZ05I & $\begin{array}{l}\text { Ex|2a: c. I748A > G } \\
\text { p.Lys583Arg / ExI3: } \\
\text { c.2027delC p.T676TfsXII }\end{array}$ & & $\begin{array}{l}\text { Ex|2a: c. I748A > G } \\
\text { p.Lys583Arg / ExI3: } \\
\text { c.2027delC p.T676TfsXII }\end{array}$ & & \\
\hline D530 & $\begin{array}{l}\text { Ex6: c.82IT > G p.Leu274Arg } \\
\text { / Ex34: c.6579 + IG >C }\end{array}$ & $\begin{array}{l}\text { With no } \\
\text { other tissue } \\
\text { analysed, } \\
\text { unable to } \\
\text { differentiate } \\
\text { germline from } \\
\text { somatic } \\
N F / \text { mutations }\end{array}$ & $\begin{array}{l}\text { Ex6: c.82IT > G p.L274R / } \\
\text { Ex34: c.6579 + IG >C }\end{array}$ & $\begin{array}{l}\text { With no } \\
\text { other tissue } \\
\text { analysed, } \\
\text { unable to } \\
\text { differentiate } \\
\text { germline from } \\
\text { somatic } \\
N F / \text { mutations }\end{array}$ & 32 \\
\hline SC049 & $\begin{array}{l}\text { Ex3: c. } 205-2 A>G \text { / Ex } 23.2: \\
\text { c. } 4084 C>\text { T p.Arg I } 362 X\end{array}$ & & $\begin{array}{l}\text { Ex3: c. } 205-2 A>G \text { / Ex23.2: } \\
\text { c. } 4084 C>\text { T p.RI } 362 X\end{array}$ & & \\
\hline SCO87 & $\begin{array}{l}\text { Ex4b: c.482T > G p.Leul6IX } \\
\text { / Ex4b: c.495_498delTGTT } \\
\text { p.TI65TfsXII }\end{array}$ & & $\begin{array}{l}\text { Ex4b: c.482T > G p.LI6IX / } \\
\text { Ex4b: c.495_498delTGTT } \\
\text { p.TI65TfsXII }\end{array}$ & & \\
\hline D252 & $\mathrm{NI}$ & $\mathrm{NI}$ & $\begin{array}{l}\text { Ex29: c.5242C }>T \\
\text { p.Argl } 748 \times \underline{R}\end{array}$ & Nonsense & \\
\hline \multicolumn{6}{|c|}{ Glomus tumours } \\
\hline NFI-G8 & Ex4a: c.3IIT > G p.Leul04X & Nonsense & $\begin{array}{l}\text { Ex44: c. } 7727 C>A \\
\text { p.Ser } 2576 X\end{array}$ & Nonsense & 36 \\
\hline
\end{tabular}


Table S2. Continued

\begin{tabular}{|c|c|c|c|c|c|}
\hline $\begin{array}{l}\text { Patient } \\
\text { ID }\end{array}$ & $\begin{array}{l}\text { Germline point } \\
\text { mutation }\end{array}$ & $\begin{array}{l}\text { Type of } \\
\text { germline } \\
\text { mutation }\end{array}$ & $\begin{array}{l}\text { Somatic point } \\
\text { mutation }\end{array}$ & $\begin{array}{l}\text { Effect of } \\
\text { somatic } \\
\text { mutation }\end{array}$ & Source \\
\hline NFI-G3 & $\begin{array}{l}\text { ExI6: c.2546insG } \\
\text { p.Val850SerfsXI5 }\end{array}$ & $\begin{array}{l}\text { I bp insertion } \\
\text { (FS) }\end{array}$ & $\begin{array}{l}\text { Ex29: c.5539_5546dup8 } \\
\text { p.Ser I850Valfs XI5 }\end{array}$ & $\begin{array}{l}8 \text { bp } \\
\text { duplication } \\
\text { (FS) }\end{array}$ & \\
\hline NFI-G5 & Ex27a: c.45I5-2A > T & Splice site & Ex|8: c.3II3+IG >C & Splice site & \\
\hline NFI-GI & $\begin{array}{l}\text { mRNA study: Exon } 29 \\
\text { partially skipped }\end{array}$ & Splice site? & $\begin{array}{l}\text { Ex4a: c. } 403 \text { delC } \\
\text { p.Arg I } 35 \text { Glyfs X30 }\end{array}$ & $\begin{array}{l}\text { I bp deletion } \\
\text { (FS) }\end{array}$ & \\
\hline $\begin{array}{l}\mathrm{NFI}- \\
\mathrm{GIOa}\end{array}$ & $\begin{array}{l}\text { Ex37: c.6789_6792delTTAC } \\
\text { p.Tyr2264AspfsX5 }\end{array}$ & $\begin{array}{l}4 \text { bp deletion } \\
\text { (FS) }\end{array}$ & Ex2: c. $204+\mathrm{IG}>\mathrm{A}$ & Splice site & \\
\hline $\begin{array}{l}\mathrm{NFI-} \\
\mathrm{GIOb}\end{array}$ & & & $\begin{array}{l}\text { Ex43: c.7600_762I del22 } \\
\text { p.Lys2534GlyfsX8 }\end{array}$ & $\begin{array}{l}22 \text { bp deletion } \\
\text { (FS) }\end{array}$ & \\
\hline \multicolumn{6}{|l|}{ ACs } \\
\hline & $\begin{array}{l}\text { No NFI somatic mutations } \\
\text { identified }\end{array}$ & & $\begin{array}{l}\text { No NFI somatic mutations } \\
\text { identified }\end{array}$ & & \\
\hline \multicolumn{6}{|c|}{ Gastric carcinoid tumours } \\
\hline & $\begin{array}{l}\text { No NFI somatic mutations } \\
\text { identified }\end{array}$ & & $\begin{array}{l}\text { No NFI somatic mutations } \\
\text { identified }\end{array}$ & & \\
\hline \multicolumn{6}{|l|}{ PCs } \\
\hline & $\begin{array}{l}\text { No NFI somatic mutations } \\
\text { identified }\end{array}$ & & $\begin{array}{l}\text { No NFI somatic mutations } \\
\text { identified }\end{array}$ & & \\
\hline
\end{tabular}

FS, frame shift; NI, no information; R, recurrent. 


\section{Supplementary Table References}

1. Spurlock, G., Griffiths, S., Uff, J. and Upadhyaya, M. (2007), 'Somatic alterations of the NF1 gene in an NF1 individual with multiple benign tumours (internal and external) and malignant tumour types', Fam. Cancer Vol. 6, pp. 463-471.

2. De Raedt, T., Maertens, O., Chmara, M., Brems, H. et al. (2006), 'Somatic loss of wild type NF1 allele in neurofibromas: Comparison of NF1 microdeletion and non-microdeletion patients', Genes Chromosomes Cancer Vol. 45, pp. 893-904.

3. Maertens, O., Brems, H., Vandesompele, J., De Raedt, T. et al. (2006), 'Comprehensive NF1 screening on cultured Schwann cells from neurofibromas', Hum. Mutat. Vol. 27, pp. 1030-1040.

4. Thomas, L., Kluwe, L., Chuzhanova, N., Mautner, V. et al. (2010), 'Analysis of NF1 somatic mutations in cutaneous neurofibromas from patients with high tumor burden', Neurogenetics Vol. 11, pp. 391-400.

5. Serra, E., Puig, S., Otero, D., Gaona, A. et al. (1997), 'Confirmation of a double-hit model for the NF1 gene in benign neurofibromas', Am. J. Hum. Genet. Vol. 61, pp. 512-519.

6. Serra, E., Ars, E., Ravella, A., Sánchez, A. et al. (2001), 'Somatic NF1 mutational spectrum in benign neurofibromas: mRNA splice defects are common among point mutations', Hum. Genet. Vol. 108, pp. 416-429.

7. Serra, E., Rosenbaum, T., Nadal, M., Winner, U. et al. (2001), 'Mitotic recombination effects homozygosity for NF1 germline mutations in neurofibromas', Nat. Genet. Vol. 28, pp. 294-296.

8. Eisenbarth, I., Beyer, K., Krone, W. and Assum, G. (2000), 'Toward a survey of somatic mutation of the NF1 gene in benign neurofibromas of patients with neurofibromatosis type 1', Am. J. Hum. Genet. Vol. 66, pp. 393-401.

9. Rasmussen, S., Overman, J., Thomson, S., Colman, S. et al. (2000), 'Chromosome 17 loss-of-heterozygosity studies in benign and malignant tumors in neurofibromatosis type 1', Genes Chromosomes Cancer Vol. 28, pp. $425-431$.

10. Upadhyaya, M., Spurlock, G., Monem, B., Thomas, N. et al. (2008), 'Germline and somatic NF1 gene mutations in plexiform neurofibromas', Hum. Mutat. Vol. 29, pp. E103-E111.

11. Steinmann, K., Kluwe, L., Friedrich, R., Mautner, V. et al. (2009), 'Mechanisms of loss of heterozygosity in neurofibromatosis type 1-associated plexiform neurofibromas', J. Invest. Dermatol. Vol. 129, pp. 615-621.

12. Däschner, K., Assum, G., Eisenbarth, I., Krone, W. et al. (1997), 'Clonal origin of tumor cells in a plexiform neurofibroma with LOH in NF1 intron 38 and in dermal neurofibromas without LOH of the NF1 gene', Biochem. Biophys. Res. Commun. Vol. 234, pp. 346-350.

13. Frahm, S., Mautner, V., Brems, H., Legius, E. et al. (2004), 'Genetic and phenotypic characterization of tumor cells derived from malignant peripheral nerve sheath tumors of neurofibromatosis type 1 patients', Neurobiol. Dis. Vol. 16, pp. 85-91.

14. Kluwe, L., Friedrich, R. and Mautner, V. (1999), 'Allelic loss of the NF1 gene in NF1-associated plexiform neurofibromas', Cancer Genet. Cytogenet. Vol. 113, pp. 65-69.

15. De Luca, A., Buccino, A., Gianni, D., Mangino, M. et al. (2003), 'NF1 gene analysis based on DHPLC', Hum. Mutat. Vol. 21, pp. 171-172.

16. Upadhyaya, M., Spurlock, G., Kluwe, L., Chuzhanova, N. et al. (2009), 'The spectrum of somatic and germline NF1 mutations in NF1 patients with spinal neurofibromas', Neurogenetics Vol. 10, pp. 251-263.

17. Upadhyaya, M., Kluwe, L., Spurlock, G., Monem, B. et al. (2008), 'Germline and somatic NF1 gene mutation spectrum in NF1-associated malignant peripheral nerve sheath tumors (MPNSTs)', Hum. Mutat. Vol. 29, pp. $74-82$.

18. Bottillo, I., Ahlquist, T., Brekke, H., Danielsen, S. et al. (2009), 'Germline and somatic NF1 mutations in sporadic and NF1-associated malignant peripheral nerve sheath tumours', J. Pathol. Vol. 217, pp. 693-701.

19. Skuse, G., Kosciolek, B. and Rowley, P. (1989), 'Molecular genetic analysis of tumors in von Recklinghausen neurofibromatosis: Loss of heterozygosity for chromosome 17', Genes Chromosomes Cancer Vol. 1, pp. $36-41$.
20. Menon, A., Anderson, K., Riccardi, V., Chung, R. et al. (1990), 'Chromosome $17 \mathrm{p}$ deletions and p53 gene mutations associated with the formation of malignant neurofibrosarcomas in von Recklinghausen neurofibromatosis', Proc. Natl. Acad. Sci. USA Vol. 87, pp. 5435-5439.

21. Glover, T., Stein, C., Legius, E., Andersen, L. et al. (1991), 'Molecular and cytogenetic analysis of tumors in von Recklinghausen neurofibromatosis', Genes Chromosomes Cancer Vol. 3, pp. 62-70.

22. Xu, W., Mulligan, L.M., Ponder, M.A., Liu, L. et al. (1992), 'Loss of NF1 alleles in phaeochromocytomas from patients with type I neurofibromatosis', Genes Chromosomes Cancer Vol. 4, pp. 337-342.

23. Legius, E., Marchuk, D., Collins, F. and Glover, T. (1993), 'Somatic deletion of the neurofibromatosis type 1 gene in a neurofibrosarcoma supports a tumour suppressor gene hypothesis', Nat. Genet. Vol. 3, pp. 122-126.

24. Lothe, R., Slettan, A., Saeter, G., Brøgger, A. et al. (1995), 'Alterations at chromosome 17 loci in peripheral nerve sheath tumors', J. Neuropathol. Exp. Neurol. Vol. 54, pp. 65-73.

25. Upadhyaya, M., Han, S., Consoli, C., Majounie, E. et al. (2004), 'Characterization of the somatic mutational spectrum of the neurofibromatosis type 1 (NF1) gene in neurofibromatosis patients with benign and malignant tumors', Hum. Mutat. Vol. 23, pp. 134-146.

26. Gutmann, D., Donahoe, J., Brown, T., James, C. et al. (2000), 'Loss of neurofibromatosis 1 (NF1) gene expression in NF1-associated pilocytic astrocytomas', Neuropathol. Appl. Neurobiol. Vol. 26, pp. 361-367.

27. Kluwe, L., Hagel, C., Tatagiba, M., Thomas, S. et al. (2001), 'Loss of NF1 alleles distinguish sporadic from NF1-associated pilocytic astrocytomas', J. Neuropathol. Exp. Neurol. Vol. 60, pp. 917-920.

28. Gutmann, D., James, C., Poyhonen, M., Louis, D. et al. (2003), 'Molecular analysis of astrocytomas presenting after age 10 in individuals with NF1', Neurology Vol. 61, pp. 1397-1400.

29. Stewart, W., Traynor, J.P., Cooke, A., Griffiths, S. et al. (2007), 'Gastric carcinoid: Germline and somatic mutation of the neurofibromatosis type 1 Gene', Fam. Cancer Vol. 6, pp. 147-152.

30. Stewart, D., Corless, C., Rubin, B., Heinrich, M. et al. (2007), 'Mitotic recombination as evidence of alternative pathogenesis of gastrointestinal stromal tumours in neurofibromatosis type 1', J. Med. Genet. Vol. 44, p. e61.

31. Flotho, C., Steinemann, D., Mullighan, C., Neale, G. et al. (2007), 'Genome-wide single-nucleotide polymorphism analysis in juvenile myelomonocytic leukemia identifies uniparental disomy surrounding the NF1 locus in cases associated with neurofibromatosis but not in cases with mutant RAS or PTPN11', Oncogene Vol. 26, pp. 5816-5821.

32. Stephens, K., Weaver, M., Leppig, K., Maruyama, K. et al. (2006), 'Interstitial uniparental isodisomy at clustered breakpoint intervals is a frequent mechanism of NF1 inactivation in myeloid malignancies', Blood Vol. 108, pp. 1684-1689.

33. Steinemann, D., Arning, L., Praulich, I., Stuhrmann, M. et al. (2010), 'Mitotic recombination and compound-heterozygous mutations are predominant NF1-inactivating mechanisms in children with juvenile myelomonocytic leukemia and neurofibromatosis type 1', Haematologica Vol. 95, pp. 320-323.

34. Gutmann, D.H., Cole, J.L., Stone, W.J., Ponder, B.A. et al. (1994), 'Loss of neurofibromin in adrenal gland tumors from patients with neurofibromatosis type I', Genes Chromosomes Cancer Vol. 10, pp. 55-58.

35. Bausch, B., Borozdin, W., Mautner, V.F., Hoffmann, M.M. et al. (2007), 'Germline NF1 mutational spectra and loss-of-heterozygosity analyses in patients with pheochromocytoma and neurofibromatosis type 1', J. Clin. Endocrinol. Metab. Vol. 92, pp. 2784-2792.

36. Brems, H., Park, C., Maertens, O., Pemov, A. et al. (2009), 'Glomus tumors in neurofibromatosis type 1: Genetic, functional, and clinical evidence of a novel association', Cancer Res. Vol. 69, pp. 7393-7401.

37. Wiest, V., Eisenbarth, I., Schmegner, C., Krone, W. et al. (2003), 'Somatic NF1 mutation spectra in a family with neurofibromatosis type 1: Toward a theory of genetic modifiers', Hum. Mutat. Vol. 22, pp. 423-427.

38. Sawada, S., Florell, S., Purandare, S., Ota, M. et al. (1996), 'Identification of NF1 mutations in both alleles of a dermal neurofibroma', Nat. Genet. Vol. 14, pp. 110-112.

39. John, A., Ruggieri, M., Ferner, R. and Upadhyaya, M. (2000), 'A search for evidence of somatic mutations in the NF1 gene', J. Med. Genet. Vol. 37, pp. 44-49. 